\title{
MODEL ASSISTED VARIABLE CLUSTERING: MINIMAX-OPTIMAL RECOVERY AND ALGORITHMS
}

\author{
By Florentina Bunea \\ Cornell University \\ By Christophe Giraud \\ CNRS, Université Paris Sud, Université Paris-Saclay \\ BY XI LUO \\ Brown University \\ By Martin Royer \\ CNRS, Université Paris Sud, Université Paris-Saclay \\ AND \\ By NiCOLAS VERZELEN \\ INRA, Supagro, Univ. Montpellier
}

\begin{abstract}
The problem of variable clustering is that of estimating groups of similar components of a $p$-dimensional vector $X=\left(X_{1}, \ldots, X_{p}\right)$ from $n$ independent copies of $X$. There exists a large number of algorithms that return data-dependent groups of variables, but their interpretation is limited to the algorithm that produced them. An alternative is model-based clustering, in which one begins by defining population level clusters relative to a model that embeds notions of similarity. Algorithms tailored to such models yield estimated clusters with a clear statistical interpretation. We take this view here and introduce the class of $G$-block covariance models as a background model for variable clustering. In such models, two variables in a cluster are deemed similar if they have similar associations will all other variables. This can arise, for instance, when groups of variables are noise corrupted versions of the same latent factor. We quantify the difficulty of clustering data generated from a $G$-block covariance model in terms of cluster proximity, measured with respect to two related, but different, cluster separation metrics. We derive minimax cluster separation thresholds, which are the metric values below which no algorithm can recover the model-defined clusters exactly, and show that they are different for the two metrics. We therefore develop two
\end{abstract}

MSC 2010 subject classifications: Primary 62H30; secondary 62C20

Keywords and phrases: convergence rates, convex optimization, covariance matrices, high dimensional inference

1

imsart-aos ver. 2014/10/16 file: mainSupparXiv.tex date: December 14, 2018 
algorithms, COD and PECOK, tailored to $G$-block covariance models, and study their minimax-optimality with respect to each metric. Of independent interest is the fact that the analysis of the PECOK algorithm, which is based on a corrected convex relaxation of the popular $K$-means algorithm, provides the first statistical analysis of such algorithms for variable clustering. Additionally, we compare our methods with another popular clustering method, spectral clustering. Extensive simulation studies, as well as our data analyses, confirm the applicability of our approach.

1. Introduction. The problem of variable clustering is that of grouping similar components of a $p$-dimensional vector $X=\left(X_{1}, \ldots, X_{p}\right)$. These groups are referred to as clusters. In this work we investigate the problem of cluster recovery from a sample of $n$ independent copies of $X$. Variable clustering has had a long history in a variety of fields, with important examples stemming from gene expression data $[41,19,23]$ or protein profile data [8]. The solutions to this problem are typically algorithmic and entirely data based. They include applications of $K$-means, hierarchical clustering, spectral clustering, or versions of them. The statistical properties of these procedures have received a very limited amount of investigation. It is not currently known what probabilistic cluster models on $X$ can be estimated by these popular techniques, or by their modifications. More generally, modelbased variable clustering has received a limited amount of attention. One net advantage of model-based clustering is that population-level clusters are clearly defined, offering both interpretability of the clusters and a benchmark against which one can check the quality of a particular clustering algorithm.

In this work we propose the $G$-block covariance model as a flexible model for variable clustering and show that the clusters given by this model are uniquely defined. We then motivate and develop two algorithms tailored to the model, COD and PECOK, and analyze their respective performance in terms of exact cluster recovery, for minimally separated clusters, under appropriately defined cluster separation metrics.

1.1. The G-block covariance model. Our proposed model for variable clustering subsumes that the covariance matrix $\Sigma$ of a centered random vector $X \in \mathbf{R}^{p}$ follows a block, or near-block, decomposition, with blocks corresponding to a partition $G=\left\{G_{1}, \ldots, G_{K}\right\}$ of $\{1, \ldots, p\}$. This structure of the covariance matrix has been observed to hold, empirically, in a number of very recent studies on the parcelation of the human brain, for instance $[25,20,18,40]$. We further support these findings in Section 7 , where we apply the clustering methods developed in this paper, tailored to $G$-block

imsart-aos ver. 2014/10/16 file: mainSupparXiv.tex date: December 14, 2018 
covariance models, for the clustering of brain regions.

To describe our model, we associate, to a partition $G$, a membership matrix $A \in \mathbf{R}^{p \times K}$ defined by $A_{a k}=1$ if $a \in G_{k}$, and $A_{a k}=0$ otherwise.

(A) The exact $G$-block covariance model. In view of the above discussion, clustering the variables $\left(X_{1}, \ldots, X_{p}\right)$ amounts to find a minimal (i.e. coarsest partition) $G^{*}$, such that two variables belong to the same cluster if they have the same covariance with all other variables. This implies that the covariance matrix $\Sigma$ of $X$ decomposes as

$$
\Sigma=A C^{*} A^{t}+\Gamma,
$$

where $A$ is relative to $G^{*}, C^{*}$ is a symmetric $K \times K$ matrix, and $\Gamma$ a diagonal matrix. When a such a decomposition exists with the partition $G^{*}$, we say that $X \in \mathbf{R}^{p}$ follows an (exact) $G^{*}$-block covariance model.

(i) $G$-Latent Model. Such a structure arises, for instance, when components of $X$ that belong to the same group can be decomposed into the sum between a common latent variable and an uncorrelated random fluctuation. Similarity within group is therefore given by association with the same unobservable source. Specifically, the exact block-covariance model (1.1) holds, with a diagonal matrix $\Gamma$, when

$$
X_{a}=Z_{k(a)}+E_{a}
$$

with $\operatorname{Cov}\left(Z_{k(a)}, E_{a}\right)=0, \operatorname{Cov}(Z)=C^{*}$, and the individual fluctuations $E_{a}$ are uncorrelated, and thus $E$ has diagonal covariance matrix $\Gamma$. The index assignment function $k:\{1, \ldots, p\} \rightarrow\{1, \ldots, K\}$ is defined by $G_{k}=$ $\{a: k(a)=k\}$. In practice, this model is used to justify the construction of a single variable that represents a cluster, the average of $X_{a}, a \in G_{k}$, viewed as an observable proxy of $Z_{k(a)}$. For example, a popular analysis approach for fMRI data, called region-of-interest (ROI) analysis [36], requires averaging the observations from multiple voxels (a imaging unit for a small cubic volume of the brain) within each ROI (or cluster of voxels) to produce new variables, each representing a larger and interpretable brain area. These new variables are then used for downstream analyses. From this perspective, model (1.2) can be used in practice, see, for example [7], as a building block in a data analysis based on cluster representatives, which in turn requires accurate cluster estimation. Indeed, data-driven methods for clustering either voxels into regions or regions into functional systems, especially based on the covariance matrix of $X$, is becoming increasingly important, see for example $[20,40,18,37]$. Accurate data-driven clustering

imsart-aos ver. 2014/10/16 file: mainSupparXiv.tex date: December 14, 2018 
methods also enable studying the cluster differences across subjects [17] or experimental conditions [22].

(ii) The Ising Block Model. The Ising Block Model has been proposed in [9] for modelling social interactions, for instance political affinities. Under this model, the joint distribution of $X \in\{-1,1\}^{p}$, a $p$-dimensional vector with binary entries, is given by

$$
f(x)=\frac{1}{\kappa_{\alpha, \beta}} \exp \left[\frac{\beta}{2 p} \sum_{a \sim b} x_{a} x_{b}+\frac{\alpha}{2 p} \sum_{a \nsim b} x_{a} x_{b}\right],
$$

where the quantity $\kappa_{\alpha, \beta}$ is a normalizing constant, and the notation $a \sim b$ means that the elements are in the same group of the partition. The variables $X_{a}$ may for instance represent the votes of U.S. senators on a bill [6]. For parameters $\alpha>\beta$, the density (1.3) models the fact that senators belonging to the same political group tend to share the same vote. By symmetry of the density $f$, the covariance matrix $\Sigma$ of $X$ decomposes as an exact block covariance model $\Sigma=A C^{*} A^{t}+\Gamma$ where $\Gamma$ is diagonal. When all groups $G_{k}^{*}$ have identical size, we have $C^{*}=\left(\omega_{\text {in }}-\omega_{\text {out }}\right) I_{K}+\omega_{\text {out }} J$ and $\Gamma=\left(1-\omega_{\text {in }}\right) I$, where the $K \times K$ matrix $J$ has all entries equal to 1 , and $I_{K}$ denotes the $K \times K$ identity matrix, and the quantities $\omega_{i n}, \omega_{\text {out }}$ depend on $\alpha, \beta, p$.

(B) The approximate $G$-block model. In many situations, it is more appealing to group variables that nearly share the same covariance with all the other variables. In that situation, the covariance matrix $\Sigma$ would decompose as

$$
\Sigma=A C A^{t}+\Gamma \text {, where } \Gamma \text { has small off-diagonal entries. }
$$

Such a situation can arise, for instance when $X_{a}=\left(1+\delta_{a}\right) Z_{k(a)}+E_{a}$, with $\delta_{a}=o(1)$ and the individual fluctuations $E_{a}$ are uncorrelated, $1 \leq a \leq p$.

1.2. Our contribution. We assume that the data consist in i.i.d. observations $X^{(1)}, \ldots, X^{(n)}$ of a random vector $X$ with mean 0 and covariance matrix $\Sigma$. This work is devoted to the development of computationally feasible methods that yield estimates $\widehat{G}$ of $G^{*}$, such that $\widehat{G}=G^{*}$, with high probability, when the clusters are minimally separated, and to characterize the minimal value of the cluster separation from a minimax perspective. The separation between clusters is a key element in quantifying the difficulty of a clustering task as, intuitively, well separated clusters should be easier to identify. We consider two related, but different, separation metrics, that can be viewed as canonical whenever $\Sigma$ satisfies (1.4). Although all our results 
allow, and are proved, for small departures from the diagonal structure of $\Gamma$ in (1.1), our main contribution can be best seen when $\Gamma$ is a diagonal matrix. We focus on this case below, for clarity of exposition. The case of $\Gamma$ being a perturbation of a diagonal matrix is treated in Section 6 .

When $\Gamma$ is diagonal, our target partition $G^{*}$ can be easily defined. It is the unique minimal (with respect to partition refinement) partition $G^{*}$ for which there is a decomposition $\Sigma=A C^{*} A^{t}+\Gamma$, with $A$ associated to $G^{*}$. We refer to Section 2 for details. We observe in particular, that $\max _{c \neq a, b}\left|\Sigma_{a c}-\Sigma_{b c}\right|>0$ if and only if $X_{a}$ and $X_{b}$ belong to different clusters in $G^{*}$.

This last remark motivates our first metric MCOD based on the following COvariance Difference (COD) measure

$$
\operatorname{COD}(a, b):=\max _{c \neq a, b}\left|\Sigma_{a c}-\Sigma_{b c}\right| \text { for any } a, b=1, \ldots, p .
$$

We use the notation $a \stackrel{G^{*}}{\sim} b$ whenever $a$ and $b$ belong to the same group $G_{k}^{*}$, for some $k$, in the partition $G^{*}$, and similarly $a \stackrel{G^{*}}{\nsim} b$ means that there does not exist any group $G_{k}^{*}$ of the partition $G^{*}$ that contains both $a$ and $b$. We define the MCOD metric as

$$
\operatorname{MCOD}(\Sigma):=\min _{\substack{G^{*} \\ a \nsim b}} \operatorname{COD}(a, b)
$$

The measure $\operatorname{COD}(a, b)$ quantifies the similarity of the covariances that $X_{a}$ and $X_{b}$ have, respectively, with all other variables. From this perspective, the size of $\operatorname{MCOD}(\Sigma)$ is a natural measure for the difficulty of clustering when analyzing clusters with components that are similar in this sense. Moreover, note that this metric is well defined even if $C^{*}$ of model (1.1) is not semi-positive definite.

Another cluster separation metric appears naturally when we view model (1.1) as arising via model (1.2), or via small deviations from it. Then, clusters in (1.1) are driven by the latent factors, and intuitively they differ when the latent factors differ. Specifically, we define the "within-between group" covariance gap

$$
\Delta\left(C^{*}\right):=\min _{j<k}\left(C_{k k}^{*}+C_{j j}^{*}-2 C_{j k}^{*}\right)=\min _{j<k} \mathbf{E}\left[\left(Z_{j}-Z_{k}\right)^{2}\right],
$$

where the second equality holds whenever (1.2) holds. In the latter case, the matrix $C^{*}$, which is the covariance matrix of the latent factors, is necessarily semi-positive definite. Further, we observe that $\Delta\left(C^{*}\right)=0$ implies $Z_{j}=Z_{k}$ a.s. Conversely, we prove in Corollary 2.3 of Section 2 that if the decomposition (1.1) holds with $\Delta\left(C^{*}\right)>0$, then the partition related to $A$ is the

imsart-aos ver. 2014/10/16 file: mainSupparXiv.tex date: December 14, 2018 
partition $G^{*}$ described above. An instance of $\Delta\left(C^{*}\right)>0$ corresponds to having the within group covariances stronger than those between groups. This suggests the usage of this metric $\Delta\left(C^{*}\right)$ for cluster analysis whenever, in addition to the general model formulation (1.1), we also expect clusters to have this property, which has been observed, empirically, to hold in applications. For instance, it is implicit in the methods developed by [18] for creating a human brain atlas by partitioning appropriate covariance matrices. We also present a neuroscience-based data example in Section 7.

Formally, the two metrics are connected via the following chain of inequalities, proved in Lemma B.1 of Section 2 of the supplementary material [13], and valid as soon as the size of the smallest cluster is larger than one, $\Gamma$ and $C^{*}$ is semi-positive definite (for the last inequality)

$$
2 \lambda_{K}\left(C^{*}\right) \leq \Delta\left(C^{*}\right) \leq 2 \operatorname{MCOD}(\Sigma) \leq 2 \sqrt{\Delta\left(C^{*}\right)} \max _{k=1, \ldots, K} \sqrt{C_{k k}^{*}} .
$$

The first inequality shows that conditions on $\Delta\left(C^{*}\right)$ are weaker than conditions on the minimal eigenvalue $\lambda_{K}\left(C^{*}\right)$ of $C^{*}$. In order to preserve the generality of our model, we do not necessarily assume that $\lambda_{K}\left(C^{*}\right)>0$, as we show that, for model identifiability, it is enough to have the weaker condition $\Delta\left(C^{*}\right)>0$, when the two quantities differ.

The second inequality in (1.8) shows that $\Delta\left(C^{*}\right)$ and $\operatorname{MCOD}(\Sigma)$ can have the same order of magnitude, whereas the third inequality shows that they can also differ in order, and $\Delta\left(C^{*}\right)$ can be as small as $\operatorname{MCOD}^{2}(\Sigma)$, for small values of these metrics, which is our main focus. This suggests that different statistical assessments, and possibly different algorithms, should be developed for estimators of clusters defined by (1.1), depending on the cluster separation metric. To substantiate this intuition, we first derive, for each metric, the rate below which no algorithm can recover exactly the clusters defined by (1.1). We call this the minimax optimal threshold for cluster separation, and prove that it is different for the two metrics. We call an algorithm that can be proved to recover exactly clusters with separation above the minimax threshold a minimax optimal algorithm.

Theorem 3.1 in Section 3 shows that, for $K \geq 3$ and for some numerical constant $c>0$, no algorithm can estimate consistently clusters defined by (1.1) uniformly over covariance matrices fulfilling

$$
\operatorname{MCOD}(\Sigma) \geq c \sqrt{\frac{\log (p)}{n}}
$$

Theorem 3.2 in Section 3 shows that optimal separation distances with respect to the metric $\Delta\left(C^{*}\right)$ are sensitive to the size of the smallest cluster,

$$
m^{*}=\min _{1 \leq k \leq K}\left|G_{k}^{*}\right| .
$$

imsart-aos ver. 2014/10/16 file: mainSupparXiv.tex date: December 14, 2018 
Indeed, there exists a numerical constant $c>0$, such that no algorithm can estimate consistently clusters defined by (1.1) uniformly over covariance matrices fulfilling

$$
\Delta\left(C^{*}\right) \geq c\left(\sqrt{\frac{\log (p)}{n m^{*}}} \bigvee \frac{\log (p)}{n}\right) .
$$

The first term will be dominant whenever the smallest cluster has size $m^{*}<$ $n / \log (p)$, which will be the case in most situations. The second term in (1.9) becomes dominant whenever $m^{*}>n / \log (p)$, which can also happen when $p$ scales as $n$, and we have a few balanced clusters.

The PECOK algorithm is tailored to the $\Delta\left(C^{*}\right)$ metric, and is shown in Theorem 5.3 to be near-minimax optimal. For instance, for balanced clusters, there exists a constant $c^{\prime}$ such that exact recovery is guaranteed when $\Delta\left(C^{*}\right) \geq c^{\prime}\left(\sqrt{\frac{K \vee \log p}{m^{*} n}}+\frac{K \vee \log (p)}{n}\right)$. This differs by factors in $K$ from the $\Delta\left(C^{*}\right)$-minimax threshold, for general $K$, whereas it is of optimal order when $K$ is a constant, or grows as slowly as $\log p$. A similar discrepancy between minimax lower bounds and the performance of polynomial-time estimators has also been pinpointed in network clustering via the stochastic block model [16] and in sparse PCA [10]. It has been conjectured that, when $K$ increases with $n$, there exists a gap between the statistical boundary, i.e. the minimal cluster separation for which a statistical method achieves perfect clustering with high probability, and the polynomial boundary, i.e. the minimal cluster separation for which there exists a polynomial-time algorithm that achieves perfect clustering. Further investigation of this computational trade-off is beyond the scope of this paper and we refer to [16] and [10] for more details.

However, if we consider directly the metric $\operatorname{MCOD}(\Sigma)$, and its corresponding, larger, minimax threshold, we derive the COD algorithm, which is minimax optimal with respect to $\operatorname{MCOD}(\Sigma)$ when $K \geq 3$. In view of (1.8), it is also minimax optimal with respect to $\Delta\left(C^{*}\right)$, whenever there exist small clusters, the size of which does not change with $n$. The description of the two algorithms and theoretical properties are given in Sections 4 and 5, respectively, for exact block covariance models. Companions of these results, regarding the performance of the algorithms for approximate block covariance models are given in Section 6, in Theorem 6.2 and Theorem 6.5, respectively.

Table 1 below gives a snap-shot of our results, which for ease of presentation, correspond to the case of balanced clusters, with the same number of variables per cluster. We stress that neither our algorithms, nor our theory,

imsart-aos ver. 2014/10/16 file: mainSupparXiv.tex date: December 14, 2018 
is restricted to this case, but the exposition becomes more transparent in this situation.

\begin{tabular}{|c|c|c|c|}
\hline Metric & $\begin{array}{l}\text { Minimax } \\
\text { threshold }\end{array}$ & PECOK & COD \\
\hline$d_{1}=: \Delta\left(C^{*}\right)$ & $\sqrt{\frac{\log p}{m n}}+\frac{\log p}{n}$ & $\begin{array}{lr}\operatorname{Minimax} & \text { optimal } \\
\text { w.r.t. } \quad d_{1} & \text { when } \\
K=O(\log (p)) & \end{array}$ & $\begin{array}{l}\text { Minimax } \\
\text { w.r.t. } d_{1} \text { when } m \text { is } \\
\text { constant. }\end{array}$ \\
\hline$d_{2}=: \operatorname{MCOD}(\Sigma)$ & $\begin{array}{l}\sqrt{\frac{\log p}{n}} \\
\text { when } K \geq 3\end{array}$ & $\begin{array}{l}\text { Minimax } \\
\text { w.r.t. } \quad d_{2} \quad \text { when } \\
m \quad>\quad n / \log (p) \text { and } \\
K=O(\log p) .\end{array}$ & $\begin{array}{l}\text { Minimax optimal } \\
\text { w.r.t. } d_{2} \text { when } K \geq 3 \text {. }\end{array}$ \\
\hline
\end{tabular}

Algorithm performance relative to minimax thresholds of each metric

In this table $m$ denotes the size of the smallest cluster in the partition. The performance of COD under $d_{1}$ follows from the second inequality in (1.8), whereas the performance of PECOK under $d_{2}$ follows from the last inequality in (1.8). The overall message transmitted by Table 1 and our analysis is that, irrespective of the separation metric, the COD algorithm will be most powerful whenever we expect to have at least one, possibly more, small clusters, a situation that is typically not handled well in practice by most of the popular clustering algorithms, see [12] for an in-depth review. The PECOK algorithm is expected to work best for larger clusters, in particular when there are no clusters of size one. We defer more comments on the relative numerical performance of the methods to the discussion Section 8.3 .

We emphasize that both our algorithms are generally applicable, and our performance analysis is only in terms of the most difficult scenarios, when two different clusters are almost indistinguishable and yet, as our results show, consistently estimable. Our extensive simulation results confirm these theoretical findings.

We summarize below our key contributions.

(1) An identifiable model for variable clustering and metrics for cluster separation. We advocate model-based variable clustering, as a way of proposing objectively defined and interpretable clusters. We propose identifiable $G$-block covariance models for clustering, and prove cluster identifiability in Proposition 2.2 of Section 2.

(2) Minimax lower bounds on cluster separation metrics for exact partition recovery. Two of our main results are Theorem 3.2 and Theorem 
3.1, presented in Section 3, in which we establish, respectively, minimax limits on the size of the $\Delta\left(C^{*}\right)$-cluster separation and $\operatorname{MCOD}(\Sigma)$-cluster separation below which no algorithm can recover clusters defined by (1.1) consistently, from a sample of size $n$ on $X$. To the best of our knowledge these are the first results of this type in variable clustering.

(3) Variable clustering procedures with guaranteed exact recovery of minimally separated clusters. The results of (1) and (2) provide a much needed framework for motivating variable clustering algorithm development and for clustering algorithm assessments.

In particular, they motivate a correction of a convex relaxation of the $K$-means algorithm, leading to our proposed PECOK procedure, based on Semi-Definite Programing (SDP). Theorem 5.3 shows it to be near-minimax optimal with respect to the $\Delta\left(C^{*}\right)$ metric. The PECOK - $\Delta\left(C^{*}\right)$ pairing is natural, as $\Delta\left(C^{*}\right)$ measures the difference of the "within cluster" signal relative to the "between clusters" signal, which is the idea that underlies $K$-means type procedures. To the best of our knowledge, this is the first work that explicitly shows what model-based clusters of variables can be estimated via $K$-means style methods, and assesses theoretically the quality of estimation. Moreover, our work shows that the results obtained in [9], for the block Ising model, can be generalized to arbitrary values of $K$ and unbalanced clusters.

The COD procedure is a companion of PECOK for clusters given by model (1.1), and is minimax optimal with respect to the $\operatorname{MCOD}(\Sigma)$ cluster separation when $K \geq 3$, as established in Theorem 3.1. Another advantage of COD is of computational nature, as SDP-based methods, although convex, can be computationally involved.

(4) Comparison with corrected spectral variable clustering methods. In Section 5.4, we connect PECOK with another popular algorithm, spectral clustering. Spectral clustering is less computationally involved than PECOK, but the theoretical guaranties that we can offer for it are weaker.

1.3. Organization of the paper. The rest of the paper is organized as follows:

Sections 1.4 and 1.5 contain the notation and distributional assumptions used throughout the paper.

For clarity of exposition, Sections 2 - 5 contain results established for model (1.1), when is $\Gamma$ a diagonal matrix. Extensions to the case when $\Gamma$ has small off-diagonal entries are presented in Section 6.

Section 2 shows that we have a uniquely defined target of estimation, the partition $G^{*}$.

imsart-aos ver. 2014/10/16 file: mainSupparXiv.tex date: December 14, 2018 
Section 3 derives the minimax thresholds on the separation metrics $\Delta\left(C^{*}\right)$ and $M C O D(\Sigma)$, respectively, for estimating $G^{*}$ consistently.

Section 4 is devoted to the COD algorithm, and its analysis.

Section 5 is devoted to the PECOK algorithm and its analysis.

Section 5.4 analyses spectral clustering for variable clustering, and compares it with PECOK.

Section 6 contains extensions to approximate $G$-block covariance models.

Section 7 presents their application to the clustering of putative brain areas using a real fMRI data.

Section 8 contains a discussion of our results and overall recommendations regarding the usage of our methods. Given the space constraints, all proofs and simulation results are included in the supplementary material.

The implementation of PECOK can be found at http://github.com/ martinroyer/pecok/ and that of COD at http://CRAN.R-project.org/ package $=$ cord.

1.4. Notation. We denote by $\mathbf{X}$ the $n \times p$ matrix with rows corresponding to observations $X^{(i)} \in \mathbf{R}^{p}$, for $i=1, \ldots, n$. The sample covariance matrix $\widehat{\Sigma}$ is defined by

$$
\widehat{\Sigma}=\frac{1}{n} \mathbf{X}^{t} \mathbf{X}=\frac{1}{n} \sum_{i=1}^{n} X^{(i)}\left(X^{(i)}\right)^{t} .
$$

Given a vector $v$ and $q \geq 1,|v|_{q}$ stands for the $\ell_{q}$ norm. For a generic matrix $M:|M|_{q}$ denotes its the entry-wise $\ell_{q}$ norm, $\|M\|_{o p}$ denotes its operator norm, and $\|M\|_{F}$ refers to the Frobenius norm. We use $M_{: a}, M_{b:}$, to denote the $a$-th column or, respectively, $b$-th row of a generic matrix $M$. The bracket $\langle.,$.$\rangle refers to the Frobenius scalar product. Given a matrix M$, we denote $\operatorname{supp}(M)$ its support, that is the set of indices $(i, j)$ such that $M_{i j} \neq 0$. $I$ denotes the identity matrix. We define the variation semi-norm of a diagonal matrix $D$ as $|D|_{V}:=\max _{a} D_{a a}-\min _{a} D_{a a}$. We use $B \succcurlyeq 0$ to denote a symmetric and positive semidefinite matrix.

Throughout this paper will make use of the notation $c_{1}, c_{2}, \cdots$ to denote positive constants independent of $n, p, K, m$. The same letter, for instance $c_{1}$ may be used in different statements and may denote different constants, which are made clear within each statement, when there is no possibility for confusion.

We use $[p]$ to denote the set $\{1, \ldots, p\}$. We use the notation $a \stackrel{G}{\sim} b$ whenever $a, b \in G_{k}$, for the same $k$. Also, $m=\min _{k}\left|G_{k}\right|$ stands for the size of the smallest group of the partition $G$.

imsart-aos ver. 2014/10/16 file: mainSupparXiv.tex date: December 14, 2018 
The notation $\gtrsim$ and $\lesssim$ is used for whenever the inequalities hold up to multiplicative numerical constants.

1.5. Distributional assumptions. For a $p$-dimensional random vector $Y$, its Orlicz norm is defined by $\|Y\|_{\psi_{2}}=\sup _{t \in \mathbf{R}^{p}:\|t\|_{2}=1} \inf \left\{s>0: \mathbb{E}\left[e^{\left(Z^{t} t\right) / s^{2}} \leq\right.\right.$ $2]\}$. Throughout the paper we will assume that $X$ follows a sub-Gaussian distribution. Specifically, we use:

Assumption 1. (sub-Gaussian distributions) The exists $L>0$ such that random vector $\Sigma^{-1 / 2} X$ satisfies $\left\|\Sigma^{-1 / 2} X\right\|_{\psi_{2}} \leq L$, where

Our class of distributions includes, in particular, that of bounded distributions, which may be of independent interest, as example (ii) illustrates. We will therefore also specialize some of our results to this case, in which case we will use directly

Assumption 1-bis. (Bounded distributions) There exists $M>0$ such that $\max _{i=1, \ldots, p}\left|X_{i}\right| \leq M$ almost surely.

Gaussian distributions satisfy Assumption 1 with $L=1$. A bounded distribution is also sub-Gaussian, but the corresponding quantity $L$ can be much larger than $M$, and sharper results can be obtained if Assumption 1-bis holds.

2. Cluster identifiability in $G$-block covariance models. To keep the presentation focused, we consider in sections 2-5 the model (1.1) with $\Gamma$ diagonal. We treat the case corresponding to a diagonally dominant $\Gamma$ in Section 6 below. In the sequel, it is assumed that $p>2$.

We observe that if the decomposition (1.1) holds for a partition $G$, it also holds for any sub-partition of $G$. It is natural therefore to seek the smallest (coarsest) of such partitions, that is the partition with the least number of groups for which (1.1) holds. Since the partition ordering is a partial order, the smallest partition is not necessarily unique. However, the following lemma shows that uniqueness is guaranteed for our model class.

Lemma 2.1. Consider any covariance matrix $\Sigma$.

(a) There exists a unique minimal partition $G^{*}$ such that $\Sigma=A C A^{t}+\Gamma$ for some diagonal matrix $\Gamma$, some membership matrix $A$ associated to $G^{*}$ and some matrix $C$.

(b) The partition $G^{*}$ is given by the equivalence classes of the relation

$$
a \equiv b \quad \text { if and only if } \operatorname{COD}(a, b):=\max _{c \neq a, b}\left|\Sigma_{a c}-\Sigma_{b c}\right|=0 .
$$

Proof of Lemma 2.1. If decomposition $\Sigma=A C A^{t}+\Gamma$ holds with $A$ related to a partition $G$, then we have $C O D(a, b)=0$ for any $a, b$ belonging

imsart-aos ver. 2014/10/16 file: mainSupparXiv.tex date: December 14, 2018 
to the same group of $G$. Hence, each group $G_{k}$ of $G$ is included in one of the equivalence class of $\equiv$. As a consequence, $G$ is a finer partition than $G^{*}$ as defined in (b). Hence, $G^{*}$ is the (unique) minimal partition such that decomposition $\Sigma=A C A^{t}+\Gamma$ holds.

As a consequence, the partition $G^{*}$ is well-defined and is identifiable. Next, we discuss the definitions of MCOD and $\Delta$ metrics. For any partition $G$, we let $\operatorname{MCOD}(\Sigma, G):=\min _{a{ }_{a} b} \operatorname{COD}(a, b)$, where we recall that the notation $a \stackrel{G}{\nsim} b$ means that $a$ and $b$ are not in a same group of the partition $G$. By definition of $G^{*}$, we notice that $\operatorname{MCOD}\left(\Sigma, G^{*}\right)>0$ and the next proposition shows that $G^{*}$ is characterized by this property.

Proposition 2.2. Let $G$ be any partition such that $\operatorname{MCOD}(\Sigma, G)>0$ and the decomposition $\Sigma=A C A^{t}+\Gamma$ holds with $A$ associated to $G$. Then $G=G^{*}$.

The proofs of this proposition and the following corollary are given in Section B of the supplementary material [13]. In what follows, we use the notation $\operatorname{MCOD}(\Sigma)$ for $\operatorname{MCOD}\left(\Sigma, G^{*}\right)$.

In general, without further restrictions on the model parameters, the decomposition $\Sigma=A C A^{t}+\Gamma$ with $A$ relative to $G^{*}$ is not unique. If, for instance $\Sigma$ is the identity matrix $I$, then $G^{*}$ is the complete partition (with $p$ groups) and the decomposition (1.1) holds for any $(C, \Gamma)=(\lambda I,(1-\lambda) I)$ with $\lambda \in \mathbf{R}$.

Recall that $m^{*}:=\min \left|G_{k}^{*}\right|$ stands for the size of the smallest cluster. If we assume that $m^{*}>1$ (no singleton), then $\Gamma$ is uniquely defined. Besides, the matrix $C$ in (1.1) is only defined up to a permutation of its rows and columns. In the sequel, we denote $C^{*}$ any of these matrices $C$. When the partition contains singletons $\left(m^{*}=1\right)$, the matrix decomposition $\Sigma=A C A^{t}+\Gamma$ is made unique (up to a permutation of row and columns of $C$ ) by putting the additional constraint that the entries $\Gamma_{a a}$ corresponding to singletons are equal to 0 . Since the definition of $\Delta(C)$ is invariant with respect to permutation of rows and columns, this implies that $\Delta\left(C^{*}\right)$ is well-defined for any covariance matrix $\Sigma$.

For arbitrary $\Sigma, \Delta\left(C^{*}\right)$ is not necessarily positive. Nevertheless, if $\Delta\left(C^{*}\right)>$ 0 , then $G^{*}$ is characterized by this property.

Corollary 2.3. Let $G$ be a partition such that $m=\min _{k}\left|G_{k}\right| \geq 2$, the decomposition $\Sigma=A C A^{t}+\Gamma$ holds with $A$ associated to $G$ and $\Delta(C)>0$. Then $G=G^{*}$. 
As pointed in (1.7), in the latent model (1.2), $\Delta\left(C^{*}\right)$ is equal to the square of the minimal $L^{2}$-norm between two latent variables. So, in this case, the condition $\Delta\left(C^{*}\right)>0$ simply requires that all latent variables are distincts.

\section{Minimax thresholds on cluster separation for perfect recov-} ery. Before developing variable clustering procedures, we begin by assessing the limits of the size of each of the two cluster separation metrics below which no algorithm can be expected to recover the clusters perfectly. We denote by $m^{*}=\min _{k}\left|G_{k}^{*}\right|$ the size of the smallest cluster of the target partition $G^{*}$ defined above. For $1 \leq m \leq p / 2$ and $\eta>0$, we define $\mathcal{M}(m, \eta)$ as the set of covariance matrices $\Sigma$ fulfilling $\operatorname{MCOD}(\Sigma)>\eta|\Sigma|_{\infty}$ and whose associated partition $G^{*}$ has groups of equal size $m^{*} \geq m$. Similarly, for $\tau>0$, we define $\mathcal{D}(m, \tau)$ as the set of covariance matrices $\Sigma$ fulfilling $\Delta\left(C^{*}\right)>\tau|\Gamma|_{\infty}$ and whose associated partition $G^{*}$ has groups of equal size $m^{*} \geq m$. We use the notation $\mathbb{P}_{\Sigma}$ to refer to the normal distribution with covariance $\Sigma$.

THEOREM 3.1. There exists a positive constant $c_{1}$ such that, for any $1 \leq m \leq p / 3$ and any $\eta$ such that

$$
0 \leq \eta<\eta^{*}:=c_{1} \sqrt{\frac{\log (p)}{n}},
$$

we have $\inf _{\widehat{G}} \sup _{\Sigma \in \mathcal{M}(m, \eta)} \mathbb{P}_{\Sigma}\left(\widehat{G} \neq G^{*}\right) \geq 1 / 7$, where the infimum is taken over all possible estimators.

When $2 \leq m=p / 2$, the same result holds but with the Condition (3.1) replaced by

$$
0 \leq \eta<\eta^{*}:=c_{1}\left[\sqrt{\frac{\log (p)}{n p}} \bigvee \frac{\log (p)}{n}\right]
$$

We also have:

THEOREM 3.2. There exist positive constants $c_{1}-c_{3}$ such that the following holds for any $2 \leq m \leq p / 2$. For any $\tau$ such that

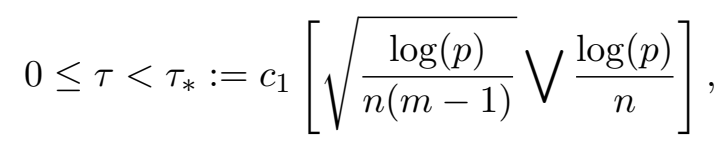

then $\inf _{\widehat{G}} \sup _{\Sigma \in \mathcal{D}(m, \tau)} \mathbb{P}_{\Sigma}\left[\widehat{G} \neq G^{*}\right] \geq 1 / 7$, where the infimum is taken over all estimators.

imsart-aos ver. 2014/10/16 file: mainSupparXiv.tex date: December 14, 2018 
Conversely, there exists a procedure $\widehat{G}$ satisfying $\sup _{\Sigma \in \mathcal{D}(m, \tau)} \mathbb{P}_{\Sigma}[\widehat{G} \neq$ $\left.G^{*}\right] \leq c_{3} / p$ for any $\tau$ such that

$$
\tau>\tau^{*}:=c_{2}\left[\sqrt{\frac{\log (p)}{n(m-1)}} \bigvee \frac{\log (p)}{n}\right]
$$

Theorems 3.2 and 3.1 show that if either metric falls below the thresholds in (3.3) and (3.1) or (3.2), respectively, the estimated partition $\widehat{G}$, irrespective of the method of estimation, cannot achieve perfect recovery with high-probability uniformly over the set $\mathcal{M}(m, \eta)$ or $\mathcal{D}(m, \tau)$. The proofs are given in Sections $\mathrm{C}$ of the supplement [13]. We note that the $\Delta\left(C^{*}\right)$ minimax threshold takes into account the size $m^{*}$ of the smallest cluster, and therefore the required cluster separation becomes smaller for large clusters. This is not the case for the second metric, as soon as there are at least 3 groups. The proof of (3.1) provides an example where we have $K=3$ clusters, that are very large, of size $m^{*}=p / 3$ each, and where the $\operatorname{MCOD}(\Sigma)$ threshold does not decrease with $m^{*}$.

Theorem 3.2 also provides a matching upper bound for the minimax threshold. Unfortunately, the procedure achieving this bound has an exponential computational complexity (see Section C.3 in the supplementary material [13] for further details and Section 5 for a near-minimax optimal algorithm with polynomial computational complexity).

\section{COD for variable clustering.}

4.1. COD Procedure. We begin with a procedure that can be viewed as natural for model (1.1). It is based on the following intuition. Two indices $a$ and $b$ belong to the same cluster of $G^{*}$, if and only if $\operatorname{COD}(a, b)=0$, with COD defined in (2.1). Equivalently, $a$ and $b$ belong to the same cluster when

$s \operatorname{COD}(a, b)=: \max _{c \neq a, b} \frac{\left|\operatorname{cov}\left(X_{a}-X_{b}, X_{c}\right)\right|}{\sqrt{\operatorname{var}\left(X_{b}-X_{a}\right) \operatorname{var}\left(X_{c}\right)}}=\max _{c \neq a, b}\left|\operatorname{cor}\left(X_{a}-X_{b}, X_{c}\right)\right|=0$,

where $s$ COD stands for scaled COvariance Differences. In the following we work with this quantity, as it is scale invariant. It is natural to place $a$ and $b$ in the same cluster when the estimator $\widehat{s \operatorname{COD}}(a, b)$ is below a certain threshold, where

$$
\begin{aligned}
& \widehat{s \operatorname{COD}}(a, b):=\max _{c \neq a, b}\left|\widehat{\operatorname{cor}}\left(X_{a}-X_{b}, X_{c}\right)\right|=\max _{c \neq a, b}\left|\frac{\widehat{\Sigma}_{a c}-\widehat{\Sigma}_{b c}}{\sqrt{\left(\widehat{\Sigma}_{a a}+\widehat{\Sigma}_{b b}-2 \widehat{\Sigma}_{a b}\right) \widehat{\Sigma}_{c c}}}\right| . \\
& \text { imsart-aos ver. 2014/10/16 file: mainSupparXiv.tex date: December 14, } 2018
\end{aligned}
$$


We estimate the partition $\widehat{G}$ according to the simple COD algorithm explained below. The algorithm does not require as input the specification of the number $K$ of groups, which is automatically estimated by our procedure. Step 3(c) of the algorithm is called the "or" rule, and can be replaced with the "and" rule below, without changing the theoretical properties of our algorithm,

$$
\widehat{G}_{l}=\left\{j \in S: \widehat{s \operatorname{COD}}\left(a_{l}, j\right) \vee \widehat{s \operatorname{COD}}\left(b_{l}, j\right) \leq \alpha\right\} .
$$

The numerical performance of these two rules are also very close through simulation studies, same as we reported on a related COD procedure on correlations [14]. Due to these small differences, we will focus on the "or" rule for the sake of space. The algorithmic complexity for computing $\widehat{\Sigma}$ is $O\left(p^{2} n\right)$ and the complexity of COD is $O\left(p^{3}\right)$, so the overall complexity of our estimation procedure is $O\left(p^{2}(p \vee n)\right)$. The procedure is also valid when $\Gamma$ has very small off-diagonal entries, and the results are presented in Section 6 .

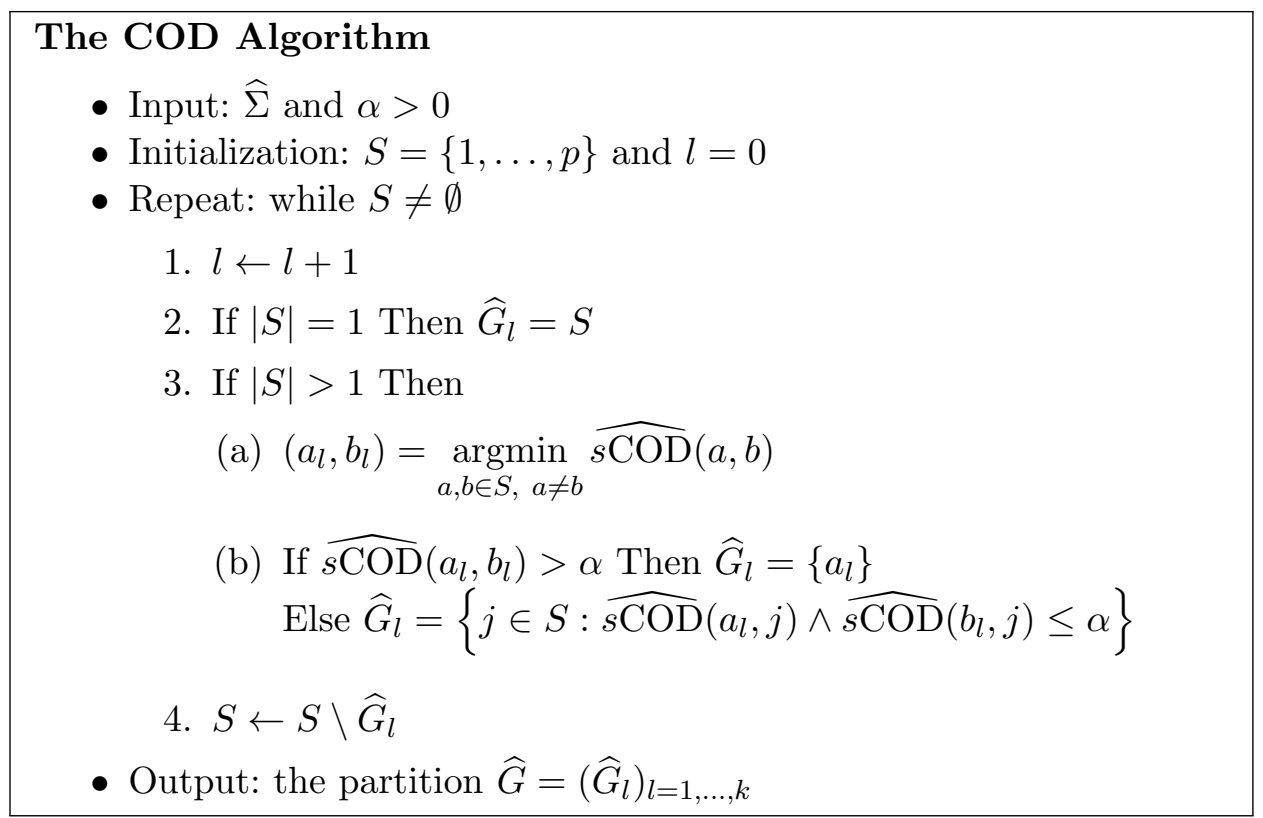

4.2. Perfect cluster recovery with COD for minimax optimal $\operatorname{MCOD}(\Sigma)$ cluster separation. Theorem 4.1 shows that the partition $\widehat{G}$ produced by the COD algorithm has the property that $\widehat{G}=G^{*}$, with high probability, as soon as the separation $\operatorname{MCOD}(\Sigma)$ between clusters exceeds a constant times the threshold (3.1) of Theorem 3.1 of the previous section.

imsart-aos ver. 2014/10/16 file: mainSupparXiv.tex date: December 14, 2018 
Theorem 4.1. Under the distributional Assumption 1, there exists numerical constants $c_{1}, c_{2}>0$ such that, if

$$
\alpha \geq c_{1} L^{2} \sqrt{\frac{\log (p)}{n}}
$$

and $\operatorname{MCOD}(\Sigma)>3 \alpha|\Sigma|_{\infty}$, then we have exact cluster recovery with probability $1-c_{2} / p$.

We recall that for Gaussian data, the constant $L=1$. The proof is given in Section D.1 of the supplement [13].

We observe that while the COD algorithm succeeds to recover $G^{*}$ at the minimax separation rate (3.1) when $K \geq 3$, it does not offer garanties at the minimax separation rate (3.2) when $K=2$. In this last case $(K=2)$, we observe that

$$
\frac{1}{2} \Delta\left(C^{*}\right) \leq \operatorname{MCOD}(\Sigma) \leq \Delta\left(C^{*}\right)
$$

so the metric $\operatorname{MCOD}(\Sigma)$ is equivalent to $\Delta\left(C^{*}\right)$ and we refer to the Section 5 for an optimal algorithm.

4.3. A Data-driven calibration procedure for COD. The performance of the COD algorithm depends on the value of the threshold parameter $\alpha$. Whereas Theorem 4.1 ensures that a good value for $\alpha$ is the order of $\sqrt{\log p / n}$, its optimal value depends on the actual distribution (at least through the subGaussian norm) and is unknown to the statistician. We propose below a new, fully data dependent, criterion for selecting $\alpha$, and the corresponding partition $\widehat{G}$, from a set of candidate partitions $\mathcal{G}$. This criterion is based on data splitting. Let us consider two independent sub-samples of the original sample, $\mathcal{D}^{1}$ and $\mathcal{D}^{1}$, each of size $n / 2$.

We denote by $\widehat{\mathcal{G}}^{(1)}$ a collection of partitions computed from $\mathcal{D}^{1}$, for instance via the COD algorithm with a varying threshold $\alpha$. For any $a<b$, we use $\mathcal{D}^{i}, i=1,2$, to calculate, respectively

$$
\widehat{\Delta}_{a b}^{(i)}=:\left[\widehat{\operatorname{Cor}}^{(i)}\left(X_{a}-X_{b}, X_{c}\right)\right]_{c \neq a, b} i=1,2 .
$$

Since $\Delta_{a b}:=\left[\operatorname{Cor}\left(X_{a}-X_{b}, X_{c}\right)\right]_{c \neq a, b}$ equals zero if and only if $a \stackrel{G}{\sim} b$, we want to select a partition $G$ such that $\widehat{\Delta}_{a b}^{(2)} \mathbf{1}_{a \nsim b}$ is a good predictor of $\Delta_{a b}$. To implement this principle, it remains to evaluate $\Delta_{a b}$ independently of $\widehat{\Delta}_{a b}^{(2)}$. For this evaluation, we propose to re-use sample $\mathcal{D}^{1}$ which has already

imsart-aos ver. 2014/10/16 file: mainSupparXiv.tex date: December 14, 2018 
been used to build the family of partitions $\widehat{\mathcal{G}}^{(1)}$. More precisely, we select $\widehat{G} \in \widehat{\mathcal{G}}^{(1)}$ by minimizing the data-splitting criterion $\mathcal{H}$ :

$$
\widehat{G} \in \underset{G \in \widehat{\mathcal{G}}^{(1)}}{\operatorname{argmin}} \mathcal{H}(G) \quad \text { with } \quad \mathcal{H}(G)=: \sum_{a<b}\left[\left|\widehat{\Delta}_{a b}^{(2)} \mathbf{1}_{a \nsim b}^{G}-\widehat{\Delta}_{a b}^{(1)}\right|_{\infty}^{2}\right] .
$$

The following proposition assesses the performance of $\widehat{G}$. We need the following additional assumption.

(P1) If $\operatorname{Cor}\left(X_{a}-X_{b}, X_{c}\right)=0$ then $\mathbb{E} \widehat{\operatorname{Cor}}\left(X_{a}-X_{b}, X_{c}\right)=0$.

In general, the sample correlation is not an unbiased estimator of the population level correlation. Still, $(\mathbf{P 1})$ is satisfied when the data are normally distributed or in a latent model (1.2) when the noise variables $E_{a}$ have a symmetric distribution. The next proposition provides guaranties for criterion $\mathcal{H}$, averaged over $\mathcal{D}^{2}$, and denoted by $\mathbb{E}^{(2)}[\mathcal{H}(G)]$. The proof is given in Section D.2 of the supplement [13].

Proposition 4.2. Assume that the distributional Assumption 1 and (P1) hold. Then, there exists a constant $c_{1}>0$ such that, when $\operatorname{MCOD}(\Sigma)>$ $c_{1}|\Sigma|_{\infty} L^{2} \sqrt{\log (p) / n}$, we have

$$
\mathbb{E}^{(2)}\left[\mathcal{H}\left(G^{*}\right)\right] \leq \min _{G \in \widehat{\mathcal{G}}^{(1)}} \mathbb{E}^{(2)}[\mathcal{H}(G)]
$$

both with probability larger than $1-4 / p$ and in expectation with respect to $\mathbb{P}^{(1)}$.

Under the condition $\operatorname{MCOD}(\Sigma)>c_{1}|\Sigma|_{\infty} L^{2} \sqrt{\log (p) / n}$, Theorem 4.1 ensures that $G^{*}$ belongs to $\widehat{\mathcal{G}}^{(1)}$ with high probability, whereas (4.2) suggests that the criterion is minimized at $G^{*}$.

If we consider a data-splitting algorithm based on $\widehat{\mathrm{COD}}(a, b)$ instead of $\widehat{\operatorname{sCOD}}(a, b)$, then we can obtain a counterpart of Proposition 4.2 without requiring the additional assumption $(\mathbf{P 1})$. Still, we favor the procedure based on $\widehat{\operatorname{sCOD}}(a, b)$ mainly for its scale-invariance property.

\section{Penalized convex $K$-means: PECOK.}

5.1. PECOK Algorithm. Motivated by the fact that the COD algorithm is minimax optimal with respect to the $\operatorname{MCOD}(\Sigma)$ metric for $K \geq 3$, but not necessarily with respect to the $\Delta\left(C^{*}\right)$ metric (unless the size of the smallest cluster is constant), we propose below an alternative procedure, that adapts to this metric. Our second method is a natural extension of one of the most

imsart-aos ver. 2014/10/16 file: mainSupparXiv.tex date: December 14, 2018 
popular clustering strategies. When we view the $G$-block covariance model as arising via the latent factor representation in (i) in the Introduction, the canonical clustering approach would be via the $K$-means algorithm [30], which is NP-hard [5]. Following Peng and Wei [34], we consider a convex relaxation of it, which is computationally feasible in polynomial time. We argue below that, for estimating clusters given by (1.1), one needs to further tailor it to our model. The statistical analysis of the modified procedure is the first to establish consistency of variable clustering via $K$-means type procedures, to the best of our knowledge.

The estimator offered by the standard $K$-means algorithm, with the number $K$ of groups of $G^{*}$ known, is

$$
\widehat{G} \in \underset{G}{\operatorname{argmin}} \operatorname{crit}(\mathbf{X}, G) \quad \text { with } \quad \operatorname{crit}(\mathbf{X}, G)=\sum_{a=1}^{p} \min _{k=1, \ldots, K}\left\|\mathbf{X}_{: a}-\overline{\mathbf{X}}_{G_{k}}\right\|^{2},
$$

and $\overline{\mathbf{X}}_{G_{k}}=\left|G_{k}\right|^{-1} \sum_{a \in G_{k}} \mathbf{X}_{: a}$.

For a partition $G$, let us introduce the corresponding partnership matrix $B$ by

$$
B_{a b}= \begin{cases}\frac{1}{\left|G_{k}\right|} & \text { if } a \text { and } b \text { are in the same group } G_{k}, \\ 0 & \text { if } a \text { and } b \text { are in a different groups. }\end{cases}
$$

we observe that $B_{a b}>0$ if and only if $a \stackrel{G}{\sim} b$. In particular, there is a one-to-one correspondence between partitions $G$ and their corresponding partnership matrices. It is shown in Peng and Wei [34] that the collection of such matrices $B$ is described by the collection $\mathcal{O}$ of orthogonal projectors fulfilling $\operatorname{tr}(B)=K, B 1=1$ and $B_{a b} \geq 0$ for all $a, b$.

Theorem 2.2 in Peng and Wei [34] shows that solving the $K$-means problem is equivalent to finding the global maximum

$$
\bar{B}=\underset{B \in \mathcal{O}}{\operatorname{argmax}}\langle\widehat{\Sigma}, B\rangle,
$$

and then recovering $\widehat{G}$ from $\bar{B}$.

The set of orthogonal projectors is not convex, so, following Peng and Wei [34], we consider a convex relaxation $\mathcal{C}$ of $\mathcal{O}$ obtained by relaxing the condition " $B$ orthogonal projector", by " $B$ positive semi-definite", leading to

$$
\begin{aligned}
& \mathcal{C}:=\left\{\begin{aligned}
& \\
B \in \mathbf{R}^{p \times p}: & \bullet \sum_{a} B_{a b}=1, \forall b \\
& \bullet B_{a b} \geq 0, \forall a, b \\
& \bullet \operatorname{tr}(B)=K
\end{aligned}\right\} \cdot \\
& \text { imsart-aos ver. } 2014 / 10 / 16 \text { file: mainSupparXiv.tex date: December 14, } 2018
\end{aligned}
$$


Thus, the (uncorrected) convex relaxation of $K$-means is equivalent with finding

$$
\widetilde{B}=\underset{B \in \mathcal{C}}{\operatorname{argmax}}\langle\widehat{\Sigma}, B\rangle
$$

To assess the relevance of this estimator, we first study its behavior at the population level, when $\widehat{\Sigma}$ is replaced by $\Sigma$ in (5.5). Indeed, if the minimizer of our criterion does not recover the true partition at the population level, we cannot expect it to be consistent, even in a large sample asymptotic context (fixed $p, n$ goes to infinity). We recall that $|\Gamma|_{V}:=\max _{a} \Gamma_{a a}-\min _{a} \Gamma_{a a}$.

Proposition 5.1. Assume that $\Delta\left(C^{*}\right)>2|\Gamma|_{V} / m^{*}$. Then, $B^{*}=\operatorname{argmax}_{B \in \mathcal{O}}\langle\Sigma, B\rangle$. If $\Delta\left(C^{*}\right)>7|\Gamma|_{V} / m^{*}$, then $B^{*}=\operatorname{argmax}_{B \in \mathcal{C}}\langle\Sigma, B\rangle$.

For $\Delta\left(C^{*}\right)$ large enough, the population version of convexified $K$-means recovers $B^{*}$. The next proposition illustrates that the condition $\Delta\left(C^{*}\right)>$ $2|\Gamma|_{V} / m^{*}$ for population $K$-means is in fact necessary.

Proposition 5.2. Consider the model (1.1) with

$C^{*}=\left[\begin{array}{ccc}\alpha & 0 & 0 \\ 0 & \beta & \beta-\tau \\ 0 & \beta-\tau & \beta\end{array}\right], \quad \Gamma=\left[\begin{array}{ccc}\gamma_{+} & 0 & 0 \\ 0 & \gamma_{-} & 0 \\ 0 & 0 & \gamma_{-}\end{array}\right], \quad$ and $\quad\left|G_{1}^{*}\right|=\left|G_{2}^{*}\right|=\left|G_{3}^{*}\right|=m^{*}$.

The population maximizer $B_{\Sigma}=\operatorname{argmax}_{B \in \mathcal{O}}\langle\Sigma, B\rangle$ is not equal to $B^{*}$ as soon as $2 \tau=\Delta\left(C^{*}\right)<\frac{2}{m^{*}}|\Gamma|_{V}$.

The two propositions above are proved in Section A.1 in the supplementary material [13]. As a consequence, when $\Gamma$ is not proportional to the identity matrix, the population minimizers based on $K$-means and convexified $K$-means do not necessary recover the true partition even when the "within-between group" covariance gap is strictly positive. This undesirable behavior of $K$-means is not completely unexpected as $K$-means is a quantization algorithm which aims to find clusters of similar width, instead of "homogeneous" clusters. Hence, we need to modify it for our purpose.

This leads us to suggesting a population level correction in Proposition 5.1. Indeed, as a direct Corollary of Proposition 5.1, we have

$$
B^{*}=\underset{B \in \mathcal{C}}{\operatorname{argmin}}\langle\Sigma-\Gamma, B\rangle,
$$

as long as $\Delta\left(C^{*}\right)>0$. This suggests the following Penalized Convex $\mathbf{K}-$ means (PECOK) algorithm, in three steps. The main step 2 produces an 
estimator $\widehat{B}$ of $B$ from which we derive the estimated partition $\widehat{G}$. We summarize this below.

\section{The PECOK algorithm}

Step 1. Estimate $\Gamma$ by $\widehat{\Gamma}$.

Step 2. Estimate $B^{*}$ by $\widehat{B}=\underset{B \in \mathcal{C}}{\operatorname{argmax}}(\langle\widehat{\Sigma}, B\rangle-\langle\widehat{\Gamma}, B\rangle)$.

Step 3 . Estimate $G^{*}$ by applying a clustering algorithm to the columns of $\widehat{B}$.

The required inputs for Step 2 of our algorithm are: (i) $\widehat{\Sigma}$, the sample covariance matrix; (ii) $\widehat{\Gamma}$, the estimator produced at Step 1; and (iii) $K$, the number of groups. Our only requirement on the clustering algorithm applied in Step 3 is that it succeeds to recover the partition $G^{*}$ when applied to true partnership matrix $B^{*}$. The standard $K$-means algorithm [30] seeded with $K$ distinct centroids, kmeans $++[4]$, or any approximate $K$-means as defined in (5.13) in Section 5.4, fulfill this property.

We view the term $\langle\widehat{\Gamma}, B\rangle$ as a penalty term on $B$, with data dependent weights $\widehat{\Gamma}$. Therefore, the construction of an accurate estimator $\widehat{\Gamma}$ of $\Gamma$ is a crucial step for guaranteeing the statistical optimality of the PECOK estimator.

5.2. Construction of $\widehat{\Gamma}$. Estimating $\Gamma$ before estimating the partition itself is a non-trivial task, and needs to be done with care. We explain our estimation below and analyze it in Proposition A.10 in Section A.5. We show that this estimator of $\Gamma$ is appropriate whenever $\Gamma$ is a diagonal matrix (or diagonally dominant, with small off-diagonal entries). For any $a, b \in[p]$, define

$$
V(a, b):=\max _{c, d \in[p] \backslash\{a, b\}} \frac{\left|\left(\widehat{\Sigma}_{a c}-\widehat{\Sigma}_{a d}\right)-\left(\widehat{\Sigma}_{b c}-\widehat{\Sigma}_{b d}\right)\right|}{\sqrt{\widehat{\Sigma}_{c c}+\widehat{\Sigma}_{d d}-2 \widehat{\Sigma}_{c d}}}
$$

with the convention $0 / 0=0$. Guided by the block structure of $\Sigma$, we define

$$
b_{1}(a):=\underset{b \in[p] \backslash\{a\}}{\operatorname{argmin}} V(a, b) \quad \text { and } \quad b_{2}(a):=\underset{b \in[p] \backslash\left\{a, b_{1}(a)\right\}}{\operatorname{argmin}} V(a, b),
$$

to be two elements "close" to $a$, that is two indices $b_{1}=b_{1}(a)$ and $b_{2}=b_{2}(a)$ such that the empirical covariance difference $\widehat{\Sigma}_{b_{i} c}-\widehat{\Sigma}_{b_{i} d}, i=1,2$, is most similar to $\widehat{\Sigma}_{a c}-\widehat{\Sigma}_{a d}$, for all variables $c$ and $d$ not equal to $a$ or $b_{i}, i=1,2$. It is expected that $b_{1}(a)$ and $b_{2}(a)$ either belong to the same group as $a$,or

imsart-aos ver. 2014/10/16 file: mainSupparXiv.tex date: December 14, 2018 
belong to some "close" groups. Then, our estimator $\widehat{\Gamma}$ is a diagonal matrix, defined by

$$
\widehat{\Gamma}_{a a}=\widehat{\Sigma}_{a a}+\widehat{\Sigma}_{b_{1}(a) b_{2}(a)}-\widehat{\Sigma}_{a b_{1}(a)}-\widehat{\Sigma}_{a b_{2}(a)}, \quad \text { for } a=1, \ldots, p .
$$

Intuitively, $\widehat{\Gamma}_{a a}$ should be close to $\Sigma_{a a}+\Sigma_{b_{1}(a) b_{2}(a)}-\Sigma_{a b_{1}(a)}-\Sigma_{a b_{2}(a)}$, which is equal to $\Gamma_{a a}$ in the favorable event where both $b_{1}(a)$ and $b_{2}(a)$ belong to the same group as $a$.

In general, $b_{1}(a)$ and $b_{2}(a)$ cannot be guaranteed to belong to the same group as $a$. Nevertheless, these two surrogates $b_{1}(a)$ and $b_{2}(a)$ are close enough to $a$ so that $\left|\widehat{\Gamma}_{a a}-\Gamma_{a a}\right|$ to be at most of the order of $|\Gamma|_{\infty} \sqrt{\log (p) / n}$ in $\ell^{\infty}$-norm, as shown in Proposition A.10 in Section A.5 of the supplement material [13]. A slightly simpler estimator of $\Gamma$ was proposed in Appendix A of a previous version of this work [15], but a bound on $\left|\widehat{\Gamma}_{a a}-\Gamma_{a a}\right|$ for that estimator contains a factor proportional to $|\Sigma|_{\infty}$, which is not desirable, and can be avoided by (5.7). In the next subsection, we show that our proposed $\widehat{\Gamma}$ leads to perfect recovery of $G^{*}$, via PECOK, under minimal separation conditions.

Note that PECOK requires the knowledge of the true number $K$ of groups. When the number $K$ of groups itself is unknown, we can modify the PECOK criterion by adding a penalty term as explained in a previous version of our work [15, Sec. 4]. Alternatively, we propose in Section G of Supplement [13] selection via a simple data-splitting procedure.

5.3. Perfect cluster recovery with PECOK for near-minimax $\Delta$-cluster separation. We show in this section that the PECOK estimator recovers the clusters exactly, with high probability, at a near-minimax separation rate with respect to the $\Delta\left(C^{*}\right)$ metric.

THEOREM 5.3. There exist $c_{1}, c_{2}, c_{3}$ three positive constants such that the following holds. Let $\widehat{\Gamma}$ be any estimator of $\Gamma$, such that $|\widehat{\Gamma}-\Gamma|_{V} \leq \delta_{n, p}$ with probability $1-c_{1} / p$. Then, under Assumption 1, and when $L^{4} \log (p) \leq c_{3} n$ and

$$
\Delta\left(C^{*}\right) \geq c_{L}\left[\|\Gamma\|_{\infty}\left\{\sqrt{\frac{\log p}{m^{*} n}}+\sqrt{\frac{p}{n m^{* 2}}}+\frac{\log (p)}{n}+\frac{p}{n m^{*}}\right\}+\frac{\delta_{n, p}}{m^{*}}\right]
$$

then $\widehat{B}=B^{*}$ and $\widehat{G}=G^{*}$, with probability higher than $1-c_{1} / p$. Here, $c_{L}$ is a positive constant that only depends on $L$ in Assumption 1. In particular, if $\widehat{\Gamma}$ is the estimator (5.7), the same conclusion holds with probability higher 
than $1-c_{2} / p$ when

$$
\Delta\left(C^{*}\right) \geq c_{L}\|\Gamma\|_{\infty}\left\{\sqrt{\frac{\log p}{m^{*} n}}+\sqrt{\frac{p}{n m^{* 2}}}+\frac{\log (p)}{n}+\frac{p}{n m^{*}}\right\} .
$$

The proof is given in Section A.3 of the supplementary material [13].

Remark 1. We left the term $\delta_{n, p}$ explicit in (5.8) in order to make clear how the estimation of $\Gamma$ affects the cluster separation $\Delta\left(C^{*}\right)$ metric. Without a correction (i.e. taking $\widehat{\Gamma}=0$ ), the term $\delta_{n, p} / m^{*}$ equals $|\Gamma|_{V} / m^{*}$ which is non zero (and does not decrease in a high-sample asymptotic) unless $\Gamma$ has equal diagonal entries. This phenomenon is consistent with the population analysis in the previous subsection. Display (5.9) shows that the separation condition can be much decreased with the correction. In particular, for balanced clusters, that is when $m^{*}=\frac{p}{K}$, exact recovery is guaranteed when

$$
\Delta\left(C^{*}\right) \geq c_{L}\left[\sqrt{\frac{K \vee \log p}{m^{*} n}}+\frac{K \vee \log p}{n}\right],
$$

for an appropriate constant $c_{L}>0$. In view of Theorem 3.2 , when $m^{*} \geq$ $c p / \log (p)$ the rate is minimax optimal, since in this case $K=p / m^{*}=$ $O(\log (p))$. When $m^{*}=o(p / \log (p))$, the number $K$ of clusters grows faster than $\log (p)$, and we possibly lose a factor $K / \log (p)$ relative to the optimal rate.

As discussed in the introduction, this gap is possibly due to a computational barrier and we refer to [16] for a discussion in the related stochastic block model.

Bounded variables $X$ also follow a sub-Gaussian distribution. Nevertheless, the corresponding sub-Gaussian norm $L$ may be large and Theorem 5.3 can sometimes be improved, as in Theorem 5.4 below, proved in Section A.3 of the supplementary material [13].

THEOREM 5.4. There exist $c_{1}, c_{2}, c_{3}$ three positive constants such that the following holds. Let $\widehat{\Gamma}$ be any estimator of $\Gamma$, such that $|\widehat{\Gamma}-\Gamma|_{V} \leq \delta_{n, p}$ with probability $1-c_{1} / p$. Then, under Assumption 1-bis, and

$$
\Delta\left(C^{*}\right) \geq c_{2}\left[M\|\Gamma\|_{\infty}^{1 / 2} \sqrt{\frac{p \log (p)}{n m^{* 2}}}+M^{2} \frac{p \log (p)}{n m^{*}}+\frac{\delta_{n, p}}{m^{*}}\right] .
$$

then $\widehat{B}=B^{*}$ and $\widehat{G}=G^{*}$, with probability higher than $1-c_{1} / p$. 
When we choose $\widehat{\Gamma}$ as in (5.7), the term $\delta_{n, p} / m^{*}$ can be simplified as under Assumption 1, see Proposition A.10 in Section A.5 of the Supplement [13]. For balanced clusters, $m^{*}=\frac{p}{K}$, Condition (5.11) can be simplified in

$$
\Delta\left(C^{*}\right) \geq c_{2}\left[M\|\Gamma\|_{\infty}^{1 / 2} \sqrt{\frac{K \log (p)}{n m^{*}}}+M^{2} \frac{K \log (p)}{n}+\frac{\delta_{n, p}}{m^{*}}\right] .
$$

In comparison to (5.10), the condition does no longer depend on the subGaussian norm $L$, but the term $K \vee \log (p)$ has been replaced by $K \log (p)$.

Remark 2. For the Ising Block Model (1.3) with $K$ balanced groups, we have $M=1$ and $p=m^{*} K, C^{*}=\left(\omega_{\text {in }}-\omega_{\text {out }}\right) I_{K}+\omega_{\text {out }} J$ and $\Gamma=\left(1-\omega_{\text {in }}\right) I_{K}$. As a consequence, no diagonal correction is needed, that is we can take $\widehat{\Gamma}=0$, and since $|\Gamma|_{V}=0$, we have $\delta_{n, p}=0$. Then, for $K$ balanced groups, condition (5.11) simplifies to

$$
\left(\omega_{\text {in }}-\omega_{\text {out }}\right) \gtrsim K \sqrt{\frac{\log (p)}{n p}}+\frac{K \log (p)}{n}
$$

In the specific case $K=2$, we recover (up to numerical multiplicative constants) the optimal rate proved in [9]. Our procedure and analysis provide a generalization of these results, as they are valid for general $K$ and Theorem 5.4 also allows for unbalanced clusters.

5.4. A comparison between PECOK and Spectral Clustering. In this section we discuss connections between the PECOK algorithm introduced above and spectral clustering, a method that has become popular in network clustering.

First, we recall the premise of spectral clustering, adapted to our context. For $G^{*}$-block covariance models as (1.1), we have $\Sigma-\Gamma=A C^{*} A^{t}$. Let $U$ be the $p \times K$ matrix collecting the $K$ leading eigenvectors of $\Sigma-\Gamma$. It has been shown, see e.g. Lemma 2.1 in Lei and Rinaldo [28], that $a$ and $b$ belong to the same cluster if and only if $U_{a}=U_{b}$ : and if and only if $\left[U U^{t}\right]_{a:}=\left[U U^{t}\right]_{b:}$. When used for variable clustering, uncorrected spectral clustering consists in applying a clustering algorithm, such as $K$-means, on the rows of the $p \times K$-matrix obtained by retaining the $K$ leading eigenvectors of $\widehat{\Sigma}$.

\section{SC algorithm}

1. Compute $\widehat{V}$, the matrix of the $K$ leading eigenvectors of $\widehat{\Sigma}$

2. Estimate $G^{*}$ by applying a (rotation invariant) clustering method to the rows of $\widehat{V}$.

Arguing as in Peng and Wei [34], we have the following.

imsart-aos ver. 2014/10/16 file: mainSupparXiv.tex date: December 14, 2018 
LEMma 5.5. $S C$ algorithm is equivalent to the following algorithm: Step 1. Find $\bar{B}=\operatorname{argmax}\{\langle\widehat{\Sigma}, B\rangle: \operatorname{tr}(B)=K, I \succcurlyeq B \succcurlyeq 0\}$.

Step 2. Estimate $G^{*}$ by applying a (rotation invariant) clustering method to the rows of $\bar{B}$.

The connection between (unpenalized) PECOK and spectral clustering now becomes clear. The (unpenalized) PECOK estimator $\widetilde{B}$ defined in (5.5) involves the calculation of

$$
\widetilde{B}=\underset{B}{\operatorname{argmax}}\left\{\langle\widehat{\Sigma}, B\rangle: B 1=1, B_{a b} \geq 0, \operatorname{tr}(B)=K, B \succcurlyeq 0\right\} .
$$

Since the matrices $B$ involved in (5.12) are doubly stochastic, their eigenvalues are smaller than 1 and hence $(5.12)$ is equivalent to $\widetilde{B}=\operatorname{argmax}_{B}\{\langle\widehat{\Sigma}, B\rangle$ : $\left.B 1=1, B_{a b} \geq 0, \operatorname{tr}(B)=K, I \succcurlyeq B \succcurlyeq 0\right\}$. Note then that $\bar{B}$ can be viewed as a less constrained version of $\widetilde{B}$, in which $\mathcal{C}$ is replaced by $\overline{\mathcal{C}}=\{B: \operatorname{tr}(B)=K, I \succcurlyeq B \succcurlyeq 0\}$, where we have dropped the $p(p+1) / 2$ constraints given by $B 1=1$, and $B_{a b} \geq 0$. The proof of Lemma 5.5 shows that $\bar{B}=\widehat{V} \widehat{V}^{t}$, so, contrary to $\widehat{B}$, the estimator $\bar{B}$ is (almost surely) never equal to $B^{*}$. Below, we adapt the arguments of [28] in order to provide some guarantees for a corrected version of spectral clustering.

In view of this connection between Spectral clustering and unpenalized PECOK and of the fact that the population justification of spectral clustering deals with the spectral decomposition of $\Sigma-\Gamma$, we propose the following corrected version of the algorithm, based on $\widetilde{\Sigma}:=\widehat{\Sigma}-\widehat{\Gamma}$.

\section{CSC algorithm}

1. Compute $\widehat{U}$, the matrix of the $K$ leading eigenvectors of $\widetilde{\Sigma}:=$ $\widehat{\Sigma}-\widehat{\Gamma}$

2. Estimate $G^{*}$ by clustering the rows of $\widehat{U}$, via an $\eta$ approximation of $K$-means (5.13).

For $\eta>1$, an $\eta$-approximation of $K$-means is a clustering algorithm producing a partition $\widehat{G}$ such that

$$
\operatorname{crit}\left(\widehat{U}^{t}, \widehat{G}\right) \leq \eta \min _{G} \operatorname{crit}\left(\widehat{U}^{t}, G\right)
$$

with $\operatorname{crit}(\cdot, \cdot)$ the $K$-means criterion (5.1). Although solving $K$-means is NPHard [5], there exist polynomial time approximate $K$-means algorithms, see Kumar et al. [26]. As a consequence of the above discussion, the first step 
of CSC can be interpreted as a relaxation of the program associated to the PECOK estimator $\widehat{B}$.

To simplify the presentation of the results for CSC procedure, we assume in the following that all the groups have the same size $\left|G_{1}^{*}\right|=\ldots=\left|G_{K}^{*}\right|=$ $m^{*}=p / K$. We emphasize that this information is not required by either PECOK or CSC, or in the proof of Proposition 5.6 below. We only use it here for simplicity. We denote by $\mathcal{S}_{K}$ the set of permutations on $\{1, \ldots, K\}$ and we denote by

$$
\bar{L}\left(\widehat{G}, G^{*}\right)=\min _{\sigma \in \mathcal{S}_{K}} \sum_{k=1}^{K} \frac{\left|G_{k}^{*} \backslash \widehat{G}_{\sigma(k)}\right|}{m^{*}}
$$

the sum of the ratios of miss-assigned variables with indices in $G_{k}^{*}$. In the previous sections, we studied perfect recovery of $G^{*}$, which would correspond to $\bar{L}\left(\widehat{G}, G^{*}\right)=0$. We give below conditions under which $\bar{L}\left(\widehat{G}, G^{*}\right) \leq \rho$, for an appropriate quantity $\rho<1$. We begin with a general theorem pertaining to partial partition recovery by CSC, under a "signal-to-noise ratio" involving the smallest eigenvalue $\lambda_{K}\left(C^{*}\right)$ of $C^{*}$.

Proposition 5.6. Let $\operatorname{Re}(\Sigma)=\operatorname{tr}(\Sigma) /\|\Sigma\|_{\text {op }}$ denote the effective rank of $\Sigma$. There exist $c_{\eta, L}>0$ only depending on $\eta$ and $L$ and a numerical constant $c_{1}$ such that the following holds under Assumption 1. For any $0<\rho<1$, if

$$
\lambda_{K}\left(C^{*}\right) \geq \frac{c_{\eta, L} \sqrt{K}\|\Sigma\|_{o p}}{m^{*} \sqrt{\rho}} \sqrt{\frac{\operatorname{Re}(\Sigma) \vee \log (p)}{n}},
$$

then $\bar{L}\left(\widehat{G}, G^{*}\right) \leq \rho$, with probability larger than $1-c_{1} / p$.

The proof extends the arguments of [28], initially developed for clustering procedures in stochastic block models, to our context. Specifically, we relate the error $\bar{L}\left(\widehat{G}, G^{*}\right)$ to the noise level, quantified in this problem by $\| \widetilde{\Sigma}-$ $A C^{*} A^{t} \|_{o p}$. We then employ the results of [24] to show that this operator norm can be controlled, with high probability, which leads to the conclusion of the theorem.

As $n$ goes to infinity, the right hand side of Condition (5.14) goes to zero, and CSC is therefore consistent in a large sample asymptotic. In contrast, we emphasize that (uncorrected) SC algorithm is not consistent as can be shown by a population analysis similar to that of Proposition 5.2.

We observe that $\Delta\left(C^{*}\right) \geq 2 \lambda_{K}\left(C^{*}\right)$, so we can compare the lower bound (5.14) on $\lambda_{K}\left(C^{*}\right)$ to the lower-bound (5.10) on $\Delta\left(C^{*}\right)$. To further facilitate 
the comparison between CSC and PECOK, we discuss both the conditions and the conclusion of this theorem in the simple setting where $C^{*}=\tau I_{K}$ and $\Gamma=I_{p}$. Then, the cluster separation measures coincide up to a factor $2, \Delta\left(C^{*}\right)=2 \lambda_{K}\left(C^{*}\right)=2 \tau$.

Corollary 5.7 (Illustrative example: $C^{*}=\tau I_{K}$ and $\Gamma=I_{p}$ ). There exist three positive numerical constants $c_{\eta, L}, c_{\eta, L}^{\prime}$ and $c_{3}$ such that the following holds under Assumption 1. For any $0<\rho<1$, if

$$
\rho \geq c_{\eta, L}\left[\frac{K^{2}}{n}+\frac{K \log (p)}{n}\right] \quad \text { and } \quad \tau \geq c_{\eta, L}^{\prime}\left[\frac{K^{2}}{\rho n} \vee \frac{K}{\sqrt{\rho n m^{*}}}\right]
$$

then $\bar{L}\left(\widehat{G}, G^{*}\right) \leq \rho$, with probability larger than $1-c_{3} / p$.

Recall that, Theorem 5.3 above states that, when $\widehat{G}$ is obtained via the PECOK algorithm, and if $\tau \gtrsim \sqrt{\frac{K \vee \log p}{m^{*} n}}+\frac{\log (p) \vee K}{n}$, then $\bar{L}\left(\widehat{G}, G^{*}\right)=0$, or equivalently, $\widehat{G}=G^{*}$, with high probability. We can therefore provide the following comparison (we refer to Section G of the supplementary material [13] for a numerical comparison).

- when $\tau \gtrsim \sqrt{\frac{K \vee \log p}{m^{*} n}}+\frac{\log (p) \vee K}{n}$, and under the additional condition that $n \gtrsim(K \vee \log (p))^{2} / K$, CSC algorithm satisfies $\bar{L}\left(\widehat{G}, G^{*}\right) \leq K^{2} /(K \vee$ $\log (p))$. So, for $K=o(\log (p))$ and for a large enough sample size $n \gtrsim(\log (p))^{2} / K$, the fraction of misclassified variables by CSC is vanishing as $O(K / \log (p))$ for $\tau \gtrsim \sqrt{\frac{\log p}{m^{*} n}}+\frac{\log (p)}{n}$. This guaranty is slightly weaker than for PECOK which ensures exact recovery in this setting. This discrepancy may be an artifact of the proof technique. Very recent works $[1,31]$ (released during the reviewing process of this paper) present reconstruction error bounds tighter than those of [28], for (variants of) spectral clustering, when applied to two parameter SBMs, for network data, not the type of data analyzed in this work.

- When we move away from the case $C^{*}=\tau I_{K}$, the guaranties for CSC can degenerate. For instance, when $\Gamma=I$ and $C^{*}=\tau I_{K}+\alpha J$, with $J$ being the matrix with all entries equal to one, as in the Ising Block model discussed page 23. Notice that in this case we continue to have $\Delta\left(C^{*}\right)=2 \lambda_{K}\left(C^{*}\right)=2 \tau$. Then, for a given, fixed, value of $\rho$ and $K$ fixed, condition (5.14) requires a cluster separation at least

$$
\tau \gtrsim \frac{\alpha \sqrt{\log (p)}}{\sqrt{n \rho}},
$$

imsart-aos ver. 2014/10/16 file: mainSupparXiv.tex date: December 14, 2018 
which is independent of $m^{*}$, unlike the condition $\tau \gtrsim \sqrt{\frac{K \vee \log p}{m^{*} n}}+$ $\frac{\log (p) \vee K}{n}$ for PECOK. This unpleasant feature is induced by the inflation of $\left\|\widetilde{\Sigma}-A C^{*} A^{t}\right\|_{o p}$ with $\alpha$. Again, this weakness in the guarantees may be an artifact of the proof, which relies on the Davis-Kahan inequality for controlling the alignment between the sample eigenvectors associated with the $K$ largest eigenvalues and their population counterpart.

All the results of this section are proved in Section E of the supplement [13].

6. Approximate $G$-block covariance models. In the previous sections, we have proved that under some separation conditions, COD and PECOK procedures are able to exactly recover the partition $G^{*}$. However, in practical situations, the separation conditions may not be met. Besides, if the entries of $\Sigma$ have been modified by an infinitesimal perturbation, then the corresponding partition $G^{*}$ would consist of $p$ singletons.

As a consequence, it may be more realistic and more appealing from a practical point of view to look for a partition $G[K]$ with $K<\left|G^{*}\right|$ groups such that $\Sigma$ is close to a matrix of the form $A C A^{t}+\Gamma$ where $\Gamma$ is diagonal and $A$ is associated to $G[K]$. This is equivalent to considering a decomposition $\Sigma=A C A^{t}+\Gamma$ with $\Gamma$ non-diagonal, where the non-diagonal entries of $\Gamma$ are small. In the sequence, we write $R=\Gamma-\operatorname{Diag}(\Gamma)$ for the matrix of the off-diagonal elements of $\Gamma$ and $D=\operatorname{Diag}(\Gamma)$ for the diagonal matrix given by the diagonal of $\Gamma$.

In the next subsection, we discuss under which conditions the partition $G[K]$ is identifiable and then, we prove that COD and PECOK are able to recover these partitions.

6.1. Identifiability of approximate $G$-block covariance models. When $\Gamma$ is allowed to be not exactly equal to a diagonal matrix, we encounter a further identifiability issue, as a generic matrix $\Sigma$ may admit many decompositions $\Sigma=A C A^{t}+\Gamma$. In fact, such a decomposition holds for any membership matrix $A$ and any matrix $C$ if we define $\Gamma=\Sigma-A C A^{t}$. So we need to specify the kind of decomposition that we are looking for. For $K$ being fixed, we would like to consider the partition $G$ with $K$ clusters that maximizes the distance between goups (e.g. $\operatorname{MCOD}(\Sigma, G)$ ) while having the smallest possible noise term $|R|_{\infty}$. Unfortunately, such a partition $G$ does not necessarily exist and is not necessarily unique. Let us illustrate this situation with a simple example. 
Example. Assume that $\Sigma$ is given by $\Sigma=\left[\begin{array}{ccc}2 r & 0 & 0 \\ 0 & 2 r & 0 \\ 0 & 0 & 2 r\end{array}\right]+I_{p}$, with $r>$ 0 , with the convention that each entry corresponds to a block of size 2 . Considering partitions with 2 groups and allowing $\Gamma$ to be non diagonal, we can decompose $\Sigma$ using different partitions. For instance

$$
\Sigma=\underbrace{\left[\begin{array}{ccc}
2 r & 0 & 0 \\
0 & r & r \\
0 & r & r
\end{array}\right]}_{=A_{1} C_{1} A_{1}^{t}}+\underbrace{\left[\begin{array}{ccc}
0 & 0 & 0 \\
0 & r & -r \\
0 & -r & r
\end{array}\right]+I_{p}}_{=\Gamma_{1}}=\underbrace{\left[\begin{array}{ccc}
r & r & 0 \\
r & r & 0 \\
0 & 0 & 2 r
\end{array}\right]}_{=A_{2} C_{2} A_{2}^{t}}+\underbrace{\left[\begin{array}{ccc}
r & -r & 0 \\
-r & r & 0 \\
0 & 0 & 0
\end{array}\right]+I_{p}}_{=\Gamma_{2}} .
$$

Importantly, the two decompositions correspond to two different partitions $G_{1}$ and $G_{2}$ and both decompositions have $\left|R_{i}\right|_{\infty}=r$ and $\operatorname{MCOD}\left(\Sigma, G_{i}\right)=$ $2 r=2|R|_{\infty}$, for $i=1,2$. In addition, no decomposition $\Sigma=A C A^{t}+D+$ $R$ with associated partition in 2 groups, satisfies $\operatorname{MCOD}(\Sigma, G)>2 r$ or $|R|_{\infty}<r$. As a consequence, there is no satisfying way to define a unique partition maximizing $\operatorname{MCOD}(\Sigma, G)$, while having $|R|_{\infty}$ as small as possible. We show below that the cutoff $\operatorname{MCOD}(\Sigma, G)>2|R|_{\infty}$ is actually sufficient for partition identifiability.

For this, let us define $\mathcal{P}_{j}(\Sigma, K), j=\{1,2\}$ as the set of quadruplets $(A, C, D, R)$ such that $\Sigma=A C A^{t}+D+R$, with $A$ a membership matrix associated to a partition $G$ with $K$ groups with $\min _{k}\left|G_{K}\right| \geq j$, and $D$ and $R$ defined as above. Hence $\mathcal{P}_{1}$ corresponds to partitions without restrictions on the minimum group size. For instance, singletons are allowed. In contrast $\mathcal{P}_{2}$ only contains partitions without singletons. We define

$\rho_{1}(\Sigma, K)=\max \left\{\operatorname{MCOD}(\Sigma, G) /|R|_{\infty}:(A, C, D, R) \in \mathcal{P}_{1}(\Sigma, K)\right.$ and $G$ associated to $\left.A\right\}$, $\rho_{2}(\Sigma, K)=\max \left\{\Delta(C) /|R|_{\infty}:(A, C, D, R) \in \mathcal{P}_{2}(\Sigma, K)\right\}$.

We view $\rho_{1}$ and $\rho_{2}$ as respective measures of "purity" of the block structure of $\Sigma$.

Proposition 6.1.

(i) Assume that $\rho_{1}(\Sigma, K)>2$. Then, there exists a unique partition $G$ such that there exists a decomposition $\Sigma=A C A^{t}+\Gamma$, with $A$ associated to $G$ and $\operatorname{MCOD}(\Sigma, G)>2|R|_{\infty}$. We denote by $G_{1}[K]$ this partition.

(ii) Assume that $\rho_{2}(\Sigma, K)>8$. Then, there exists a unique partition $G$ with $\min _{k}\left|G_{k}\right| \geq 2$, such that there exists a decomposition $\Sigma=A C A^{t}+\Gamma$, with $A$ associated to $G$ and $\Delta(C)>8|R|_{\infty}$. We denote by $G_{2}[K]$ this partition. 
(iii) In addition, if both $\rho_{1}(\Sigma, K)>2$ and $\rho_{2}(\Sigma, K)>8$, then $G_{1}[K]=$ $G_{2}[K]$.

The conditions $\rho_{1}(\Sigma, K)>2$ and $\rho_{2}(\Sigma, K)>8$ are minimal for defining uniquely the partition $G_{1}[K]$. For $\rho_{1}$, this has been illustrated in the example above the proposition. For $\rho_{2}$, we provide a counter example when $\rho_{2}(\Sigma, K)=8$ in Section B.3 of the supplement [13]. The proof of Proposition 6.1. is given in Section B.2 of [13].

The conclusion of Proposition 6.1 does essentially revert to that of Proposition 2.2 of Section 2 as soon as $|R|_{\infty}$ is small enough respective to the cluster separation sizes. Denoting $K^{*}$ the number of groups of $G^{*}$, we observe that $G_{1}\left[K^{*}\right]=G^{*}$ and $G_{2}\left[K^{*}\right]=G^{*}$ if $m^{*} \geq 2$. Besides, $\rho_{1}(\Sigma, K)=$ $\rho_{2}(\Sigma, K)=0$ for $K>K^{*}$. For $K<K^{*}$ and when $G_{1}[K]$ (resp. $\left.G_{2}[K]\right)$ are well defined, then the partition $G_{1}[K]$ (resp. $G_{2}[K]$ ) is coarser than $G^{*}$. In other words, $G_{1}[K]$ is derived from $G^{*}$ by merging groups $G_{k}^{*}$ thereby increasing $\operatorname{MCOD}(\Sigma, G)$ (resp. $\Delta(C)$ ) while requiring $|R|_{\infty}$ to be small enough.

We point out that, in general, there is no unique decomposition $\Sigma=$ $A C A^{t}+\Gamma$ with $A$ associated to $G_{2}[K]$, even when $\min _{k}\left|G_{2}[K]_{k}\right| \geq 2$. Actually, it can be possible to change some entries of $C$ and $R$, while keeping $C+R, \Delta(C)$ and $|R|_{\infty}$ unchanged.

6.2. The COD algorithm for approximate G-block covariance models. We show below that the COD algorithm is still applicable if $\Sigma$ has small departures from a block structure. We set $\lambda_{\min }(\Sigma)$ for the smallest eigenvalue of $\Sigma$.

TheOREM 6.2. Under the distributional Assumption 1, there exist numerical constants $c_{1}, c_{2}>0$ such that the following holds for all $\alpha \geq c_{1} L^{2} \sqrt{\frac{\log p}{n}}$. If, for some partition $G$ and decomposition $\Sigma=A C A^{t}+R+D$, we have

$$
|R|_{\infty} \leq \frac{\lambda_{\min }(\Sigma)}{2 \sqrt{2}} \alpha \quad \text { and } \quad \operatorname{MCOD}(\Sigma, G)>3 \alpha|\Sigma|_{\infty}
$$

then $C O D$ recovers $G$ with probability higher than $1-c_{2} / p$.

The proof is given in Section D.1 of the supplement [13]. If $G$ satisfies the assumptions of Theorem 6.2, then it follows from Proposition 6.1 that $G=G_{1}[K]$ for some $K>0$. First, consider the situation where the tuning parameter $\alpha$ is chosen to be of the order $\sqrt{\log (p) / n}$. If $\operatorname{MCOD}\left(\Sigma, G^{*}\right) \geq$ $3 \alpha|\Sigma|_{\infty}$, then COD selects $G^{*}$ with high probability. If $\operatorname{MCOD}\left(\Sigma, G^{*}\right)$ is 
smaller than this threshold, then no procedure is able to recover $G^{*}$ with high probability (Theorem 3.1). Nevertheless, COD is able to recover a coarser partition $G_{1}[K]$ whose corresponding MCOD metric $\operatorname{MCOD}(\Sigma, G)$ is higher than the threshold $3 \alpha|\Sigma|_{\infty}$ and whose matrix $R$ is small enough. For larger $\alpha$, then COD recovers a coarser partition $G$ (corresponding to $G_{1}[K]$ with a smaller $K$ ) whose corresponding approximation $|R|_{\infty}$ is allowed to be larger.

6.3. The PECOK algorithm for approximate $G$-block covariance models. In this subsection, we investigate the behavior of PECOK under the approximate $G$-block models. The number $K$ of groups being fixed, we assume that $\rho_{2}(\Sigma, K)>8$ so that $G_{2}[K]$ is well defined. We shall prove that PECOK recovers $G_{2}[K]$ with high probability. By abusing the notation, we denote in this subsection $G^{*}$ for the target partition $G_{2}[K], B^{*}$ for the associated partnership matrix and $\left(A, C^{*}, D, R\right) \in \mathcal{P}_{2}(\Sigma, K)$ any decomposition of $\Sigma$ maximizing $\Delta(C) /|R|_{\infty}$.

Similarly to Proposition 5.1, we first provide sufficient conditions on $C^{*}$ under which a population version of PECOK can recover the true partition.

Proposition 6.3. If, $\Delta\left(C^{*}\right)>\frac{7|D|_{V}+2\|R\|_{o p}}{m}+3|R|_{\infty}$, then $B^{*}=\operatorname{argmin}_{B \in \mathcal{C}}\langle\Sigma, B\rangle$.

Corollary 6.4. If $\Delta\left(C^{*}\right)>3|R|_{\infty}+\frac{2\|R\|_{o p}}{m}$, then $B^{*}=\operatorname{argmin}_{B \in \mathcal{C}}\langle\Sigma-$ $D, B\rangle$.

In contrast to the exact $G$-block model, the cluster distance $\Delta\left(C^{*}\right)$ now needs to be larger than $|R|_{\infty}$ for the population version to recover the true partition. The $|R|_{\infty}$ condition is fact necessary as discussed in subsection 6.1. In comparison to the necessary conditions discussed in subsection 6.1, there is an additional $\|R\|_{o p} / m$ term. The proofs are given in Section A.2 in the supplementary material [13].

We now examine the behavior of PECOK when we specify the estimator $\widehat{\Gamma}$ to be as in (5.7). Note that in this approximate block covariance setting, the diagonal estimator $\widehat{\Gamma}$ is in fact an estimator of the diagonal matrix $D$. In order to derive deviation bounds for our estimator $\widehat{\Gamma}$, we need the following diagonal dominance assumption.

Assumption 2: (diagonal dominance of $\Gamma$ ) The matrix $\Gamma=D+R$ fulfills

$$
\left.\Gamma_{a a} \geq 3 \max _{c: c \neq a}\left|\Gamma_{a c}\right| \quad \text { (or equivalently } \quad D_{a a} \geq 3 \max _{c: c \neq a}\left|R_{a c}\right|\right) .
$$

imsart-aos ver. 2014/10/16 file: mainSupparXiv.tex date: December 14, 2018 
The next theorem states that PECOK estimator $\widehat{B}$ recovers the groups under similar conditions to that of Theorem 5.3 if $R$ is small enough. The proof is given in Section A.3 of the supplement [13].

TheOREM 6.5. There exist $c_{1}, c_{2}, c_{L}, c_{L}^{\prime}$ four positive constants such that the following holds. Under Assumptions 1 and 2 , and when $L^{4} \log (p) \leq c_{1}$ n and

$|R|_{\infty}+\frac{\sqrt{|R|_{\infty}|D|_{\infty}}+\|R\|_{o p}}{m} \leq c_{L}\|\Gamma\|_{o p}\left\{\sqrt{\frac{\log p}{m n}}+\sqrt{\frac{p}{n m^{2}}}+\frac{\log (p)}{n}+\frac{p}{n m}\right\}$

we have $\widehat{B}=B^{*}$ and $\widehat{G}=G^{*}$, with probability higher than $1-c_{2} / p$, as soon as

$$
\Delta\left(C^{*}\right) \geq c_{L}^{\prime}\left[\|\Gamma\|_{o p}\left\{\sqrt{\frac{\log p}{m n}}+\sqrt{\frac{p}{n m^{2}}}+\frac{\log (p)}{n}+\frac{p}{n m}\right\}\right],
$$

So, as a long as $|R|_{\infty}$ and $\|R\|_{o p}$ are small enough so that (6.3) are satisfied, PECOK algorithm will correctly identify the target partition $G^{*}$ at the $\Delta$-(near) optimal minimax level (6.4). A counterpart of Theorem 6.5 for Assumption 1-bis is provided in Section A.3 of the supplement [13].

7. Data analysis. Using functional MRI data, [37] found that the human brain putative areas are organized into clusters, sometimes referred to as networks or functional systems. We use a publicly available fMRI dataset to illustrate the clusters recovered by different methods. The dataset was originally published in [39] and is publicly available from Open fMRI (https : //openfmri.org/data-sets) under the accession number ds000007. We will focus on analyzing two scan sessions from subject 1 under a visualmotor stop/go task (task 1). Before performing the analysis, we follow the preprocessing steps suggested by [39], and we follow [37] to subsample the whole brain data using $p=264$ putative areas, see Section H of the supplementary materials [13] for details. This subject was also scanned in two separate sessions, and each session yielded $n=180$ samples for each putative area.

We apply our data-splitting approach described in Section 4.3 to these two session data. Using the first scan session data only, we first estimate $\hat{G}$ using COD and COD-CC on a fine grid of $\alpha=c \sqrt{\log (p) / n}$ where $c=$ $0.5,0.6, \ldots, 3$. For a fair comparison, we set $K$ in PECOK to be the same as the resulting $K$ 's found by COD. We then use the second session data to evaluate the loss $\mathcal{H}(G)$ given in Section 4.3. Among our methods (COD,

imsart-aos ver. 2014/10/16 file: mainSupparXiv.tex date: December 14, 2018 
COD-CC, and PECOK), COD yields the smallest loss when $K=142$. We thus first focus on illustrating the COD clusters here. Table 2 lists the largest cluster of putative areas recovered by COD and their functional classification based on prior knowledge. Most of these areas are classified to be related to visual, motor, and task functioning, which is consistent with the implication of our experimental task that requires the subject to perform motor responses based on visual stimuli. Figure 1(a) plots the locations of these coordinates on a standard brain template. It shows that our COD cluster appears to come mostly from approximately symmetric locations from the left and right hemisphere, though we do not enforce this brain function symmetry in our algorithm. Note that the original coordinates in [37] are not sampled with exact symmetry from both hemispheres of the brain, and thus we do not expect exact symmetric locations in the resulting clusters based on these coordinates.

Because there are no gold standards for partitioning the brain, we follow common practice and use a prediction criterion to further compare the clustering performance of different methods. For a fair comparison, we also estimate $\hat{G}$ using K-means, HC, and spectral clustering on the same resulting $K$ 's found by COD. The prediction criterion is as follows. We first compute the covariance matrices $\hat{S}_{1}$ and $\hat{S}_{2}$ from the first and second session data respectively. For a grouping estimate $\hat{G}$, we use the following loss to evaluate its performance

$$
\left\|\hat{S}_{2}-\Upsilon\left(\hat{S}_{1}, \hat{G}\right)\right\|_{F}
$$

where block averaging operator $\Upsilon(R, G)$ produces a $G$-block structured matrix based on $\hat{G}$. For any $a \in G_{k}$ and $b \in G_{k^{\prime}}$, the output matrix entry $[\Upsilon(R, G)]_{a b}$ is given by

$$
[\Upsilon(R, G)]_{a b}= \begin{cases}\left|G_{k}\right|^{-1}\left(\left|G_{k}\right|-1\right)^{-1} \sum_{i, j \in G_{k}, i \neq j} R_{i j} & \text { if } a \neq b \text { and } k=k^{\prime} \\ \left|G_{k}\right|^{-1}\left|G_{k^{\prime}}\right|^{-1} \sum_{i \in G_{k}, j \in G_{k^{\prime}}} R_{i j} & \text { if } a \neq b \text { and } k \neq k^{\prime} \\ 1 & \text { if } a=b .\end{cases}
$$

In essence, this operator smooths over the matrix entries with indices in the same group, and one may expect that such smoothing over variables in the true cluster will reduce the loss (7.1) while smoothing over different clusters will increase the loss.

Figure 1(b) compares the prediction loss values under different group sizes for each method. This shows that our data-splitting approach for COD indeed selects a value $K=142$ that is immediately next to a slightly larger one $(K=206)$, the latter having the smallest prediction loss, near the 
Fig 1: (a) Plot of the coordinates of the largest COD cluster overplayed over a standard brain template. The coordinates are shown as red balls. (b) Comparison of COD, COD-CC, PECOK, K-means, HC, and SC using the Frobenius prediction loss criterion (7.1) where the groups are estimated by these methods respectively.

(a) A brain cluster by COD

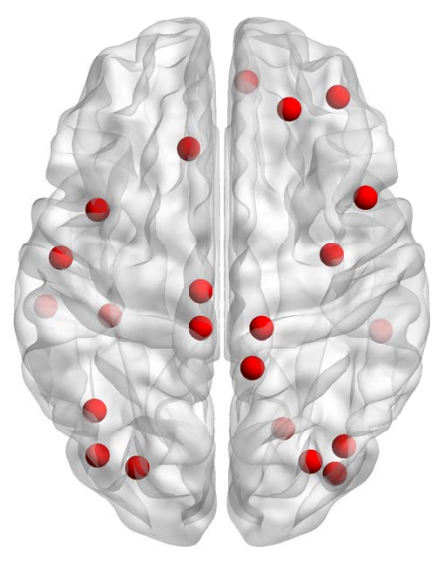

(b) Prediction loss

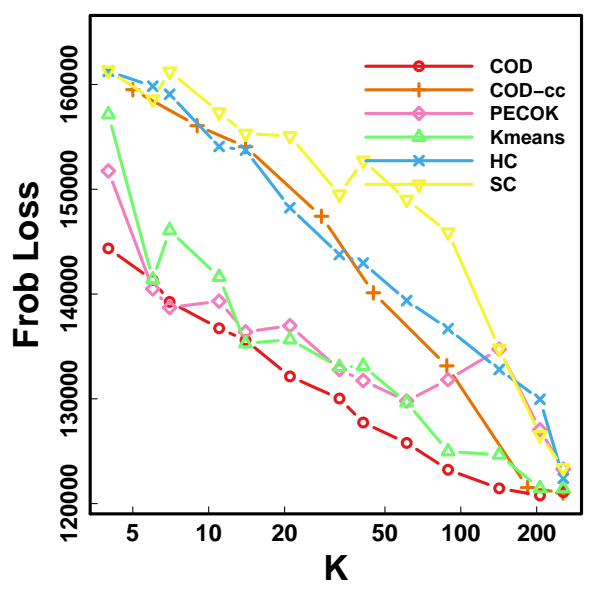

bottom plateau. However, the differences are almost negligible. This suggests that our data-splitting criterion, which comes with theoretical guarantees, also provides good prediction performance in this real data example, while selecting a slightly smaller $K$, as desired, since this makes the resulting clusters easier to describe and interpret.

Regardless of the choice of $K$ or $\alpha$, Figure 1(b) also shows that COD almost always yields the smallest prediction loss for a wide range of $K$, while PECOK does slightly better when $K$ is between 5 and 10 . Though COD-CC has large losses for medium or small $K$, its performance is very close to the best performer COD near $K=146$. Kmeans in this example is the closest competing method, while the other two methods (HC and SC) yield larger losses across the choices of $K$.

8. Discussion. In this section, we discuss some related models and give an overall recommendation on the usage of our methods.

8.1. Comparison with Stochastic Block Model. The problem of variable clustering that we consider in this work is fundamentally different from that of variable clustering from network data. The latter, especially in the 
TABLE 2

MNI coordinates $(x, y, z$, in $m m$ ) of the largest COD group and their functioning classification.

\begin{tabular}{cccccccc}
\hline $\mathrm{X}$ & $\mathrm{Y}$ & $\mathrm{Z}$ & Function & $\mathrm{X}$ & $\mathrm{Y}$ & $\mathrm{Z}$ & Function \\
\hline \hline 40 & -72 & 14 & visual & -7 & -21 & 65 & motor \\
\hline-28 & -79 & 19 & visual & -7 & -33 & 72 & motor \\
\hline 20 & -66 & 2 & visual & 13 & -33 & 75 & motor \\
\hline 29 & -77 & 25 & visual & 10 & -46 & 73 & motor \\
\hline 37 & -81 & 1 & visual & 36 & -9 & 14 & motor \\
\hline 47 & 10 & 33 & task & -53 & -10 & 24 & motor \\
\hline-41 & 6 & 33 & task & -37 & -29 & -26 & uncertain \\
\hline 38 & 43 & 15 & task & 52 & -34 & -27 & uncertain \\
\hline-41 & -75 & 26 & default & -58 & -26 & -15 & uncertain \\
\hline 8 & 48 & -15 & default & -42 & -60 & -9 & attention \\
\hline 22 & 39 & 39 & default & -11 & 26 & 25 & saliency \\
\hline
\end{tabular}

context of the Stochastic Block Model (SBM), has received a large amount of attention over the past years, for instance [21, 28, 16, 29, 2, 33, 27]. The most important difference stems from the nature of the data: the data analyzed via the SBM is a $p \times p$ binary matrix $\mathbf{A}$, called the adjacency matrix, with entries assumed to have been generated as independent Bernoulli random variables; its expected value is assumed to have a block structure. In contrast, the data matrix $\mathbf{X}$ generated from a $G$-block covariance is a $n \times p$ matrix with real entries, and rows viewed as i.i.d copies of a $p$-dimensional vector $X$ with mean zero and dependent entries. The covariance matrix $\Sigma$ of $X$ is assumed to have (up to the diagonal) a block structure.

Need for a correction. Even though the analysis of the methods in our setting would differ from the SBM setting, we could have applied available clustering procedures tailored for SBMs to the empirical covariance matrix $\widehat{\Sigma}=\mathbf{X}^{t} \mathbf{X} / n$ by treating it as some sort of weighted adjacency matrix. It turns out that applying verbatim the spectral clustering procedure of Lei and Rinaldo [28] or the SDP such as the ones in [3] would lead to poor results. The main reason for this is that, in our setting, we need to correct both the spectral algorithm and the SDP to recover the correct clusters (Section 5). Second, the SDPs studied in the SBM context (such as those of [3]) do not handle properly groups with different and unknown sizes, contrary to our SDP. To the best of our knowledge, our SDP (without correction) has only been independently studied by Mixon et al. [32] in the context of Gaussian mixtures.

Analysis of the SDP. As for the mathematical arguments, our analysis of the SDP in our on covariance-type model differs from that in mean- 
type models partly because of the the presence of non-trivial cross-product terms. Instead of relying on dual certificates arguments as in other work such as [35], we directly investigate the primal problem and combine different duality-norm bounds. The crucial step is the Lemma A.3 in the supplementary material [13] which allows to control the Frobenius inner product by a (unusual) combination of $\ell^{1}$ and spectral control. In our opinion, our approach is more transparent than dual certificates techniques, especially in the presence of a correction $\widehat{\Gamma}$ and allows for the attainment of optimal convergence rates.

8.2. Extension to other Models. The general strategy of correcting a convex relaxation of $K$-means can be applied to other models. In [38], one of the authors has adapted the PECOK algorithm to the clustering problem of mixture of subGaussian distributions. In particular, in the high-dimensional setting where the correction plays a key role, [38] obtains sharper separation conditions dependencies than in state-of-the-art clustering procedures [32]. Extensions to model-based overlapping clustering are beyond the scope of this paper, but we refer to [11] for recent results.

8.3. Practical recommendations. Based on our extensive simulation studies, we conclude this section with general recommendations on the usage of our proposed algorithms.

If $p$ is moderate in size, and if there are reasons to believe that no singletons exist in a particular application, or if they have been removed in a pre-processing step, we recommend the usage of the PECOK algorithm, which is numerically superior to existing methods: exact recovery can be reached for relatively small sample sizes. COD is also very competitive, but requires a slightly larger sample size to reach the same performance as PECOK. The constraint on the size of $p$ reflects the existing computational limits in state-of-the art algorithms for SDP, not the statistical capabilities of the procedure, the theoretical analysis of which being one of the foci of this work.

If $p$ is large, we recommend COD-type algorithms. Since COD is optimizationfree, it scales very well with $p$, and only requires a moderate sample size to reach exact cluster recovery. Moreover, COD adapts very well to data that contains singletons and, more generally, to data that is expected to have many inhomogeneous clusters.

\section{ACKNOWLEDGEMENTS}

We thank the editors and anonymous reviewers for their helpful suggestions. We thank Andrea Montanari for pointing to us the reference [32]. 
The project is partially supported by the CNRS PICS grant HighClust. Christophe Giraud is partially supported by the LabEx LMH, ANR-11LABX-0056-LMH. Martin Royer is supported by an IDEX Paris-Saclay IDI grant, ANR-11-IDEX-0003-02. Xi Luo is partially supported by NSF-DMS 1557467, NIH R01EB022911, P01AA019072, P20GM103645, P30AI042853, and S10OD016366. Florentina Bunea is partially supported by NSF-DMS 1712709 .

\section{SUPPLEMENTARY MATERIAL}

\section{" Supplement to: "Model assisted variable clustering: minimax- optimal recovery and algorithms"}

(doi: 10.1214/00-AOASXXXXSUPP; .pdf). This supplement contains: proofs of the theoretical results, the simulation results, and additional supporting information regarding the data analysis.

\section{REFERENCES}

[1] Emmanuel Abbe, Jianqing Fan, Kaizheng Wang, and Yiqiao Zhong. Entrywise eigenvector analysis of random matrices with low expected rank. arXiv preprint arXiv:1709.09565, 2017.

[2] Emmanuel Abbe and Colin Sandon. Community detection in general stochastic block models: Fundamental limits and efficient algorithms for recovery. In Foundations of Computer Science (FOCS), 2015 IEEE 56th Annual Symposium on, pages 670-688, 2015.

[3] A. A. Amini and E. Levina. On semidefinite relaxations for the block model. ArXiv e-prints, June 2014.

[4] David Arthur and Sergei Vassilvitskii. K-means++: The advantages of careful seeding. In Proceedings of the Eighteenth Annual ACM-SIAM Symposium on Discrete Algorithms, SODA '07, pages 1027-1035, Philadelphia, PA, USA, 2007. Society for Industrial and Applied Mathematics.

[5] Pranjal Awasthi, Moses Charikar, Ravishankar Krishnaswamy, and Ali Kemal Sinop. The hardness of approximation of euclidean k-means. In Symposium on Computational Geometry, 2015.

[6] Onureena Banerjee, Laurent El Ghaoui, and Alexandre d'Aspremont. Model selection through sparse maximum likelihood estimation for multivariate gaussian or binary data. Journal of Machine learning research, 9(Mar):485-516, 2008.

[7] Pierre Bellec, Vincent Perlbarg, Saâd Jbabdi, Mélanie Pélégrini-Issac, Jean-Luc Anton, Julien Doyon, and Habib Benali. Identification of large-scale networks in the brain using fmri. Neuroimage, 29(4):1231-1243, 2006.

[8] Juliana S. Bernardes, Fabio RJ Vieira, Lygia MM Costa, and Gerson Zaverucha. Evaluation and improvements of clustering algorithms for detecting remote homologous protein families. BMC Bioinformatics, 16(1):1-14, 2015.

[9] Q. Berthet, P. Rigollet, and P. Srivastava. Exact recovery in the ising blockmodel. Annals of Statistics (to appear), page arXiv:1612.03880, 2018.

[10] Quentin Berthet and Philippe Rigollet. Complexity theoretic lower bounds for sparse principal component detection. In Shai Shalev-Shwartz and Ingo Steinwart, editors,

imsart-aos ver. 2014/10/16 file: mainSupparXiv.tex date: December 14, 2018 
Proceedings of the 26th Annual Conference on Learning Theory, volume 30 of Proceedings of Machine Learning Research, pages 1046-1066, Princeton, NJ, USA, 12-14 Jun 2013. PMLR.

[11] M. Bing, F. Bunea, Y. Ning, and M. Wegkamp. Adaptive estimation in structured factor models with applications to overlapping clustering. ArXiv e-prints, 2018.

[12] Charles Bouveyron and Camille Brunet-Saumard. Model-based clustering of highdimensional data: A review. Computational Statistics \& Data Analysis, 71:52-78, 2014.

[13] F. Bunea, C. Giraud, X. Luo, M. Royer, and N. Verzelen. Supplement to: Model assisted variable clustering: minimax-optimal recovery and algorithms. Preprint, 2018.

[14] Florentina Bunea, Christophe Giraud, and Xi Luo. Minimax optimal variable clustering in $g$-models via cord. arXiv preprint arXiv:1508.01939, 2015.

[15] Florentina Bunea, Christophe Giraud, Martin Royer, and Nicolas Verzelen. Pecok: a convex optimization approach to variable clustering. arXiv preprint arXiv:1606.05100, 2016.

[16] Yudong Chen and Jiaming Xu. Statistical-computational tradeoffs in planted problems and submatrix localization with a growing number of clusters and submatrices. Journal of Machine Learning Research, 17(27):1-57, 2016.

[17] M Chong, C Bhushan, AA Joshi, S Choi, JP Haldar, DW Shattuck, RN Spreng, and RM Leahy. Individual parcellation of resting fmri with a group functional connectivity prior. NeuroImage, 156:87-100, 2017.

[18] R Cameron Craddock, G Andrew James, Paul E Holtzheimer, Xiaoping P Hu, and Helen S Mayberg. A whole brain fmri atlas generated via spatially constrained spectral clustering. Human brain mapping, 33(8):1914-1928, 2012.

[19] N Frei, AV Garcia, J Bigeard, R Zaag, E Bueso, M Garmier, S Pateyron, ML de Tauzia-Moreau, V Brunaud, S Balzergue, J Colcombet, S Aubourg, ML Martin-Magniette, and H Hirt. Functional analysis of arabidopsisimmune-related mapks uncovers a role for mpk3 as negative regulator of inducible defences. Genome Biology, 15(6):1-22, 2014.

[20] M.F. Glasser, T.S. Coalson, E.C. Robinson, C.D. Hacker, J. Harwell, E. Yacoub, K. Ugurbil, J. Andersson, C.F. Beckmann, M. Jenkinson, S.M. Smith, and D.C. Van Essen. A multi-modal parcelation of human cerebral cortex. Nature, 536:171$178,2016$.

[21] Olivier Guédon and Roman Vershynin. Community detection in sparse networks via grothendieck's inequality. arXiv preprint arXiv:1411.4686, 2014.

[22] George Andrew James, Onder Hazaroglu, and Keith A Bush. A human brain atlas derived via n-cut parcellation of resting-state and task-based fmri data. Magnetic resonance imaging, 34(2):209-218, 2016.

[23] Daxin Jiang, Chun Tang, and Aidong Zhang. Cluster analysis for gene expression data: a survey. IEEE Transactions on Knowledge and Data Engineering, 16(11):13701386, Nov 2004.

[24] Vladimir Koltchinskii and Karim Lounici. Concentration inequalities and moment bounds for sample covariance operators. Bernoulli, to appear.

[25] Ru Kong, Jingwei Li, Nanbo Sun, Mert Sabuncu, Hesheng Liu, Andrew Schaefer, Xi-Nian Zuo, Avram Holmes, Simon Eickhoff, and Thomas Yeo. Spatial topography of individual-specific cortical networks predicts human cognition, personality and emotion. https://www.biorxiv.org/content/early/2018/01/31/213041, 2018.

[26] A. Kumar, Y. Sabharwal, and S. Sen. A simple linear time $(1+\epsilon)$-approximation algorithm for k-means clustering in any dimensions. In Foundations of Computer Science, 2004. Proceedings. 45th Annual IEEE Symposium on, pages 454-462, Oct

imsart-aos ver. 2014/10/16 file: mainSupparXiv.tex date: December 14, 2018 
2004.

[27] Can M Le, Elizaveta Levina, and Roman Vershynin. Optimization via low-rank approximation for community detection in networks. arXiv preprint arXiv:1406.0067, 2014.

[28] Jing Lei and Alessandro Rinaldo. Consistency of spectral clustering in stochastic block models. Ann. Statist., 43(1):215-237, 2015.

[29] Jing Lei and Lingxue Zhu. A generic sample splitting approach for refined community recovery in stochastic block models. arXiv preprint arXiv:1411.1469, 2014.

[30] S. Lloyd. Least squares quantization in pcm. IEEE Trans. Inf. Theor., 28(2):129-137, September 1982.

[31] $\mathrm{Yu} \mathrm{Lu}$ and Harrison H Zhou. Statistical and computational guarantees of lloyd's algorithm and its variants. arXiv preprint arXiv:1612.02099, 2016.

[32] D. G. Mixon, S. Villar, and R. Ward. Clustering subgaussian mixtures by semidefinite programming. ArXiv e-prints, February 2016.

[33] Elchanan Mossel, Joe Neeman, and Allan Sly. Consistency thresholds for binary symmetric block models. arXiv preprint arXiv:140\%.1591, 2014.

[34] Jiming Peng and Yu Wei. Approximating k-means-type clustering via semidefinite programming. SIAM J. on Optimization, 18(1):186-205, February 2007.

[35] A. Perry and A. S. Wein. A semidefinite program for unbalanced multisection in the stochastic block model. ArXiv e-prints, July 2015.

[36] Russell A Poldrack. Region of interest analysis for fmri. Social cognitive and affective neuroscience, 2(1):67-70, 2007.

[37] Jonathan D Power, Alexander L Cohen, Steven M Nelson, Gagan S Wig, Kelly Anne Barnes, Jessica A Church, Alecia C Vogel, Timothy O Laumann, Fran M Miezin, Bradley L Schlaggar, et al. Functional network organization of the human brain. Neuron, 72(4):665-678, 2011.

[38] M. Royer. Adaptive clustering through semidefinite programming. Advances in Neural Information Processing Systems (NIPS), 2017.

[39] Gui Xue, Adam R Aron, and Russell A Poldrack. Common neural substrates for inhibition of spoken and manual responses. Cerebral Cortex, 18(8):1923-1932, 2008.

[40] B.T. Yeo, F.M. Krienen, J. Sepulcre, M.R. Sabuncu, D. Lashkari, M. Hollinshead, J.L. Roffman, J.W. Smoller, L. Zöllei, J.R. Polimeni, B. Fischl, H. Liu, and R.L. Buckner. The organization of the human cerebral cortex estimated by intrinsic functional connectivity. Journal of Neurophysiology, 106:1125 -1165, 2011.

[41] R. Zaag, J-P. Tamby, C. Guichard, Z. Tariq, G. Rigaill, E. Delannoy, J-P. Renou, S. Balzergue, T. Mary-Huard, S. Aubourg, M-L. Martin-Magniette, and V. Brunaud. Gem2net: from gene expression modeling to -omics networks, a new catdb module to investigate Arabidopsis thaliana genes involved in stress response. Nucleic Acids Research, 43(Database-Issue):1010-1017, 2015. 


\title{
SUPPLEMENT TO: MODEL ASSISTED VARIABLE CLUSTERING: MINIMAX-OPTIMAL RECOVERY AND ALGORITHMS
}

\author{
By Florentina BuneA \\ Cornell University \\ By Christophe Giraud \\ CNRS, Université Paris Sud, Université Paris-Saclay \\ BY XI LuO \\ Brown University \\ By MARTIN ROYER \\ CNRS, Université Paris Sud, Université Paris-Saclay \\ AND \\ By Nicolas VERZELEN \\ INRA, Supagro, Univ. Montpellier

\begin{abstract}
The following document provides proofs of the theoretical results stated in [2]. It also contains the simulation results and additional supporting information regarding the data analysis. The numbering of the quoted lemmas, propositions, corollaries and theorems will be the same as in [2]. Results in [2] will be used without explicit reference.
\end{abstract}

\section{APPENDIX A: RESULTS FOR THE PECOK ESTIMATOR}

In order to avoid notational clutter, we write $G$ for $G^{*}$ and $m$ for $m^{*}$ for the entirety of this section.

\section{A.1. The motivation for a $K$-means correction: proof of Propositions 5.1 and 5.2 .}

A.1.1. Proofs of Proposition 5.1. The basis of this proof is the following lemma.

Lemma A.1. The collection $\mathcal{C}$ contains only one matrix whose support is included in $\operatorname{supp}\left(B^{*}\right)$, that is

$$
\mathcal{C} \cap\left\{B, \operatorname{supp}(B) \subset \operatorname{supp}\left(B^{*}\right)\right\}=\left\{B^{*}\right\}
$$

Proof. Consider any matrix $B \in \mathcal{C}$ whose support is included in $\operatorname{supp}\left(B^{*}\right)$. Since $B 1=1$, it follows that each submatrix $B_{G_{k} G_{k}}$ is symmetric doubly stochastic. Since $B_{G_{k} G_{k}}$ is also positive semidefinite, we have

$$
\operatorname{tr}\left(B_{G_{k} G_{k}}\right) \geq\left\|B_{G_{k} G_{k}}\right\|_{o p} \geq 1^{t} B_{G_{k} G_{k}} 1 /\left|G_{k}\right|=1 .
$$

As $B \in \mathcal{C}$, we have $\operatorname{tr}(B)=K$, so all the submatrices $B_{G_{k} G_{k}}$ have a unit trace. Since $\left\|B_{G_{k} G_{k}}\right\|_{o p} \geq 1$, this also enforces that $B_{G_{k} G_{k}}$ contains only one non-zero eigenvalue and that a corresponding eigenvector is the constant vector 1 . As a consequence, $B_{G_{k} G_{k}}=11^{t} /\left|G_{k}\right|$ for all $k=1, \ldots, K$ and $B=B^{*}$. 
As a consequence of Lemma A.1, in order to prove Proposition 5.1 we only need to prove that $\left\langle\Sigma, B^{*}-B\right\rangle>0$, for all $B \in \mathcal{C}(\operatorname{resp} . \mathcal{O})$ such that $\operatorname{supp}(B) \nsubseteq \operatorname{supp}\left(B^{*}\right)$.

We have

$$
\left\langle\Sigma, B^{*}-B\right\rangle=\left\langle A C^{*} A^{t}, B^{*}-B\right\rangle+\left\langle\Gamma, B^{*}-B\right\rangle .
$$

Define the $p$-dimensional vector $v$ by $v=\operatorname{diag}\left(A C^{*} A^{t}\right)$. Since $B 1=1$ for all $B \in \mathcal{C}$, we have $\left\langle v 1^{t}+1 v^{t}, B^{*}-B\right\rangle=0$. Hence, we have

$$
\begin{aligned}
\left\langle A C^{*} A^{t}, B^{*}-B\right\rangle & =\left\langle A C^{*} A^{t}-\frac{1}{2}\left(v 1^{t}+1 v^{t}\right), B^{*}-B\right\rangle \\
& =\sum_{j, k} \sum_{a \in G_{j}, b \in G_{k}}\left(C_{j k}^{*}-\frac{C_{j j}^{*}+C_{k k}^{*}}{2}\right)\left(B_{a b}^{*}-B_{a b}\right) \\
& =\sum_{j \neq k} \sum_{a \in G_{j}, b \in G_{k}}\left(\frac{C_{j j}^{*}+C_{k k}^{*}}{2}-C_{j k}^{*}\right) B_{a b} \\
& =\sum_{j \neq k}\left(\frac{C_{j j}^{*}+C_{k k}^{*}}{2}-C_{j k}^{*}\right)\left|B_{G_{j} G_{k}}\right|_{1},
\end{aligned}
$$

where $B_{G_{j} G_{k}}=\left[B_{a b}\right]_{a \in G_{j}, b \in G_{k}}$. Next lemma lower bounds $\left\langle\Gamma, B^{*}-B\right\rangle$ for $B \in \mathcal{O}$. It is stated below and proved at page 3 .

Lemma A.2. For any matrix $B$ belonging to $\mathcal{O}$ and any diagonal matrix $\Gamma$,

$$
\left\langle\Gamma, B^{*}-B\right\rangle \geq-\frac{\|\Gamma\|_{V}}{m} \sum_{k \neq j}\left|B_{G_{j} G_{k}}\right|_{1} .
$$

Hence, combining (A.1) and Lemma A.2, we obtain

$$
\left\langle\Sigma, B^{*}-B\right\rangle \geq \sum_{j \neq k}\left(\frac{C_{j j}^{*}+C_{k k}^{*}}{2}-C_{j k}^{*}-\frac{\|\Gamma\|_{V}}{m}\right)\left|B_{G_{j} G_{k}}\right|_{1},
$$

for all $B \in \mathcal{O}$. The condition $\Delta\left(C^{*}\right)>\frac{2|\Gamma|_{V}}{m}$ enforces that if $\operatorname{supp}(B) \nsubseteq \operatorname{supp}\left(B^{*}\right)$ then $\left\langle\Sigma, B^{*}-B\right\rangle>$ 0. This proves the first claim of Proposition 5.1.

To show the counterpart of this result that corresponds to replacing $\mathcal{O}$ by $\mathcal{C}$, we invoke the lemma stated below and proved at page 3 .

Lemma A.3. For any $p \times p$ symmetric matrix $S$, we have for any $B \in \mathcal{C}$

$$
\left|\left\langle S, B^{*}-B\right\rangle\right| \leq 2\left[\sum_{j \neq k}\left|B_{G_{j} G_{k}}\right|_{1}\right]\left(\frac{\|S\|_{o p}}{2 m}+3\left|B^{*} S\right|_{\infty}\right) .
$$

Define the diagonal matrix $D=\left(\max _{a} \Gamma_{a a}+\min _{a} \Gamma_{a a}\right) I / 2$. Since $\operatorname{tr}(B)=\operatorname{tr}\left(B^{*}\right)$, we have $\left\langle\Gamma, B^{*}-B\right\rangle=\left\langle\Gamma-D, B^{*}-B\right\rangle$. The matrix $S=\Gamma-D$ satisfies $\|S\|_{o p}=\|\Gamma\|_{V} / 2$ and $\left|B^{*} S\right|_{\infty} \leq$ $\|\Gamma\|_{V} /(2 m)$. Applying Lemma A.3 to $S$, we obtain

$$
\left|\left\langle\Gamma, B^{*}-B\right\rangle\right| \leq \frac{7}{2 m}\|\Gamma\|_{V}\left[\sum_{j \neq k}\left|B_{G_{j} G_{k}}\right|_{1}\right] .
$$


Hence, together with (A.1), we obtain

$$
\left\langle\Sigma, B^{*}-B\right\rangle \geq \sum_{j \neq k}\left(\frac{C_{j j}^{*}+C_{k k}^{*}}{2}-C_{j k}^{*}-\frac{7\|\Gamma\|_{V}}{2 m}\right)\left|B_{G_{j} G_{k}}\right|_{1},
$$

for all $B \in \mathcal{C}$. The condition $\Delta\left(C^{*}\right)>\frac{7|\Gamma|_{V}}{m}$ enforces that if $\operatorname{supp}(B) \nsubseteq \operatorname{supp}\left(B^{*}\right)$ then $\left\langle\Sigma, B^{*}-B\right\rangle>$ 0 , which proves the second claim of Proposition 5.1. To complete the proof of Proposition 5.1 it remains to prove the two lemmas stated above.

Proof of Lemma A.2. By definition of $B^{*}$ and since $\operatorname{tr}(B)=\operatorname{tr}\left(B^{*}\right)=K$, we have

$$
\begin{aligned}
\left\langle\Gamma, B^{*}-B\right\rangle & =\left\langle\Gamma-\left(\max _{b} \Gamma_{b b}\right) I, B^{*}-B\right\rangle=\sum_{a=1}^{p}\left(\Gamma_{a a}-\left(\max _{b} \Gamma_{b b}\right)\right)\left[\frac{1}{\left|G_{k(a)}\right|}-B_{a a}\right] \\
& \geq \sum_{a=1}^{p}-\|\Gamma\|_{V}\left[\frac{1}{\left|G_{k(a)}\right|}-B_{a a}\right]_{+}
\end{aligned}
$$

Since $B$ belongs to $\mathcal{O}$, each row sums to one and each $B_{a b}$ is either equal to 0 or to $B_{a a}$. Thus,

$$
\sum_{b \notin G_{k(a)}} B_{a b}=1-\sum_{b \in G_{k(a)}} B_{a b} \geq\left[1-\left|G_{k(a)}\right| B_{a a}\right]_{+}
$$

which implies

$$
\left[\frac{1}{\left|G_{k(a)}\right|}-B_{a a}\right]_{+} \leq \frac{1}{m} \sum_{b \notin G_{k(a)}} B_{a b}
$$

Coming back to (A.3), this gives us $\left\langle\Gamma, B^{*}-B\right\rangle \geq-\frac{\|\Gamma\|_{V}}{m} \sum_{k \neq j}\left|B_{G_{j} G_{k}}\right|_{1}$.

Proof of Lemma A.3. Observe first that $B^{*}$ is a projection matrix that induces the following decomposition of $S$.

$$
S=B^{*} S+S B^{*}-B^{*} S B^{*}+\left(I-B^{*}\right) S\left(I-B^{*}\right) .
$$

By the definition of the inner product, followed by the triangle inequality, and since $\left(I-B^{*}\right) B^{*}=0$, we further have

$$
\begin{aligned}
\left|\left\langle S, B^{*}-B\right\rangle\right| & \leq 3\left|B^{*} S\right|_{\infty}\left|B^{*}\left(B^{*}-B\right)\right|_{1}+\left|\left\langle\left(I-B^{*}\right) S\left(I-B^{*}\right), B^{*}-B\right\rangle\right| \\
& =3\left|B^{*} S\right|_{\infty}\left|B^{*}\left(B^{*}-B\right)\right|_{1}+\left|\left\langle S,\left(I-B^{*}\right) B\left(I-B^{*}\right)\right\rangle\right| .
\end{aligned}
$$

Relying on the duality of the nuclear \|\|$_{*}$ and operator \|\|$_{o p}$ norms, we have

$$
\left|\left\langle S,\left(I-B^{*}\right) B\left(I-B^{*}\right)\right\rangle\right| \leq\|S\|_{o p}\left\|\left(I-B^{*}\right) B\left(I-B^{*}\right)\right\|_{*} .
$$

We begin by bounding the nuclear norm $\left\|\left(I-B^{*}\right) B\left(I-B^{*}\right)\right\|_{*}$. Since $\left(I-B^{*}\right) B\left(I-B^{*}\right) \in \mathcal{S}^{+}$, we have

$$
\left\|\left(I-B^{*}\right) B\left(I-B^{*}\right)\right\|_{*}=\operatorname{tr}\left(\left(I-B^{*}\right) B\left(I-B^{*}\right)\right)=\left\langle I-B^{*}, B\left(I-B^{*}\right)\right\rangle=\left\langle I-B^{*}, B\right\rangle .
$$


Using the fact that the sum of each row of $B$ is 1 and $\operatorname{tr}(B)=K$, we have

$$
\begin{aligned}
\left\|\left(I-B^{*}\right) B\left(I-B^{*}\right)\right\|_{*}=\left\langle I-B^{*}, B\right\rangle & =\operatorname{tr}(B)-\sum_{k=1}^{K} \sum_{a, b \in G_{k}} \frac{B_{a b}}{\left|G_{k}\right|} \\
& =K-K+\sum_{k \neq j} \sum_{a \in G_{k}, b \in G_{j}} \frac{B_{a b}}{\left|G_{k}\right|} \\
& \leq \frac{1}{m} \sum_{k \neq j}\left|B_{G_{j} G_{k}}\right|_{1} .
\end{aligned}
$$

Next, we simplify the expression of $\left|B^{*}\left(B^{*}-B\right)\right|_{1}=\left|B^{*}(I-B)\right|_{1}$.

$$
\begin{aligned}
\left|B^{*}(I-B)\right|_{1} & =\sum_{j \neq k} \sum_{a \in G_{j}, b \in G_{k}}\left|\left(B^{*} B\right)_{a b}\right|+\sum_{k=1}^{K} \sum_{a, b \in G_{k}}\left|\left[B^{*}(I-B)\right]_{a b}\right| \\
& =\sum_{j \neq k} \sum_{a \in G_{j}, b \in G_{k}} \frac{1}{\left|G_{j}\right|} \sum_{c \in G_{j}} B_{c b}+\sum_{k=1}^{K} \sum_{a, b \in G_{k}} \frac{1}{\left|G_{k}\right|}\left|1-\sum_{c \in G_{k}} B_{c b}\right| \\
& =2 \sum_{j \neq k}\left|B_{G_{j} G_{k}}\right| 1
\end{aligned}
$$

where we used again $B 1=1$ and that the entries of $B$ are nonnegative. Gathering the above bounds together with (A.4) yields the desired result. This completes the proof of this result and of Proposition 5.1.

A.1.2. Proof of Proposition 5.2. By symmetry, we can assume that the true partition matrix $B^{*}$ is diagonal block constant. Define the partition matrix $B_{1}:=\left[\begin{array}{ccc}2 / m & 0 & 0 \\ 0 & 2 / m & 0 \\ 0 & 0 & 1 /(2 m)\end{array}\right]$ where the first two blocks are of size $m / 2$ and the the last block has size $2 m$. The construction of the matrix $B_{1}$ amounts to merging groups $G_{2}$ and $G_{3}$, and to splitting $G_{1}$ into two groups of equal size. Then,

$$
\left\langle\Sigma, B^{*}\right\rangle=\gamma_{+}+2 \gamma_{-}+m \operatorname{tr}\left(C^{*}\right), \quad\left\langle\Sigma, B_{1}\right\rangle=2 \gamma_{+}+\gamma_{-}+m \operatorname{tr}\left(C^{*}\right)-m \tau .
$$

As a consequence, $\left\langle\Sigma, B_{1}\right\rangle\left\langle\left\langle\Sigma, B^{*}\right\rangle\right.$ if and only if $\tau>\frac{\gamma_{+}-\gamma_{-}}{m}$.

A.2. Analysis of the population version under the approximate model: proofs of Proposition 6.3 and Corollary 6.4. In this subsection, we prove Proposition 6.3 and Corollary 6.4. As a consequence of Lemma A.1 above, we only need to prove that,

$$
\left\langle\Sigma, B^{*}-B\right\rangle>0, \text { for all } B \in \mathcal{C} \text { such that } \operatorname{supp}(B) \nsubseteq \operatorname{supp}\left(B^{*}\right) .
$$

We have

$$
\left\langle\Sigma, B^{*}-B\right\rangle=\left\langle A C^{*} A^{t}, B^{*}-B\right\rangle+\left\langle D, B^{*}-B\right\rangle+\left\langle R, B^{*}-B\right\rangle .
$$


Define the $p$-dimensional vector $v$ by $v=\operatorname{diag}\left(A C^{*} A^{t}\right)$. Since $B 1=1$ for all $B \in \mathcal{C}$, we have $\left\langle v 1^{t}+1 v^{t}, B^{*}-B\right\rangle=0$. Hence, we have

$$
\begin{aligned}
\left\langle A C^{*} A^{t}, B^{*}-B\right\rangle & =\left\langle A C^{*} A^{t}-\frac{1}{2}\left(v 1^{t}+1 v^{t}\right), B^{*}-B\right\rangle \\
& =\sum_{j, k} \sum_{a \in G_{j}, b \in G_{k}}\left(C_{j k}^{*}-\frac{C_{j j}^{*}+C_{k k}^{*}}{2}\right)\left(B_{a b}^{*}-B_{a b}\right) \\
& =\sum_{j \neq k} \sum_{a \in G_{j}, b \in G_{k}}\left(\frac{C_{j j}^{*}+C_{k k}^{*}}{2}-C_{j k}^{*}\right) B_{a b} \\
& =\sum_{j \neq k}\left(\frac{C_{j j}^{*}+C_{k k}^{*}}{2}-C_{j k}^{*}\right)\left|B_{G_{j} G_{k}}\right|_{1},
\end{aligned}
$$

where $B_{G_{j} G_{k}}=\left[B_{a b}\right]_{a \in G_{j}, b \in G_{k}}$. From Lemma A.3 we get

$$
\left|\left\langle D, B^{*}-B\right\rangle\right| \leq \frac{7}{2 m}|D|_{V} \sum_{j \neq k}\left|B_{G_{j} G_{k}}\right|_{1}
$$

and

$$
\left|\left\langle R, B^{*}-B\right\rangle\right| \leq\left(\frac{3}{2}|R|_{\infty}+\frac{\|R\|_{o p}}{m}\right) \sum_{j \neq k}\left|B_{G_{j} G_{k}}\right|_{1} .
$$

Combining the two last inequalities with (A.6) gives the Proposition. The Corollary follows.

A.3. Exact recovery with PECOK: approximate model. Proofs of Theorems 6.5, 5.3 and 5.4. The conclusion of Theorems 6.5 and 5.3 follows by combining the conclusion of Theorems A.4 and A.5, stated below and proved in the next subsection, with the conclusion of Proposition A.10 stated and proved in Appendix A.5 below.

Specifically: Theorem A.4, specialized to $R=0$ and coupled with (i) of Proposition A.10 proves Theorem 5.3. Theorem A.5, specialized to $R=0$ and coupled with (ii) of Proposition A.10 proves Theorem 5.4. Finally, Theorem A.4 coupled with (i) of Proposition A.10 proves Theorem 6.5. If we combine Theorem A.4 with (ii) of Proposition A.10 we obtained a version of Theorem 6.5 for bounded variables, which we do not state, for space limitations.

The following theorem examines the behavior of PECOK under the general model (1.4).

TheOREM A.4. There exist $c_{1}, \ldots, c_{3}$ three positive constants such that the following holds. Let $\widehat{\Gamma}$ be any estimator of $D$, such that $|\widehat{\Gamma}-D|_{V} \leq \delta_{n, p}$ with probability $1-c_{1} /(2 p)$. Then, under Assumption 1, and when $L^{4} \log (p) \leq c_{3} n$ and

$$
\Delta\left(C^{*}\right) \geq c_{L}\left[\|\Gamma\|_{o p}\left\{\sqrt{\frac{\log p}{m n}}+\sqrt{\frac{p}{n m^{2}}}+\frac{\log (p)}{n}+\frac{p}{n m}\right\}+\frac{\delta_{n, p}+\|R\|_{o p}}{m}+|R|_{\infty}\right],
$$

we have $\widehat{B}=B^{*}$, with probability higher than $1-c_{1} / p$.

The following theorem examines the behavior of PECOK under the general model (1.4) and when the variables are bounded. 
TheOREM A.5. There exist $c_{1}, c_{2}$ two positive constants such that the following holds. Let $\widehat{\Gamma}$ be any estimator of $D$, such that $|\widehat{\Gamma}-D|_{V} \leq \delta_{n, p}$ with probability $1-c_{1} /(2 p)$. Then, under Assumption 1-bis, and when

$$
\Delta\left(C^{*}\right) \geq c_{2}\left[M\|\Gamma\|_{o p}^{1 / 2} \sqrt{\frac{p \log (p)}{n m^{2}}}+M^{2} \frac{p \log (p)}{n m}+\frac{\delta_{n, p}+\|R\|_{o p}}{m}+|R|_{\infty}\right],
$$

we have $\widehat{B}=B^{*}$, with probability higher than $1-c_{1} / p$.

A.3.1. Proofs of Theorems A.4 and A.5. In contrast to other SDP analyses performed for other models [8], our proof does not rely on dual certificates techniques. Instead of that, we directly investigate the primal problem and combine different duality-norm bounds. In our opinion, this makes the arguments more transparent. The two key ingredients are Lemmas A.1 and A.3 above.

Given $k, l \in[K]$, we define $\Delta_{k l}\left(C^{*}\right)=C_{k k}^{*}+C_{l l}^{*}-2 C_{k l}^{*}$. As a consequence of Lemma A.1 page 1 , we only need to prove that

$$
\left\langle\widehat{\Sigma}-\widehat{\Gamma}, B^{*}-B\right\rangle>0, \text { for all } B \in \mathcal{C} \text { such that } \operatorname{supp}(B) \nsubseteq \operatorname{supp}\left(B^{*}\right),
$$

with high probability.

We begin by introducing some notation. For any $k, l \in[K]$, we denote $m_{k}=\left|G_{k}\right|$ the size of group $G_{k}$ and

$$
\gamma_{k l}=\frac{1}{m_{k} m_{l}} \sum_{a \in G_{k}, b \in G_{l}} \Gamma_{a b}
$$

Recall that $\mathbf{X}$ denotes the $n \times p$ matrix of observations and we set $\underline{\mathbf{Z}}=\mathbf{X} A^{t}\left(A^{t} A\right)^{-1}$. We have the decomposition

$$
\mathbf{X}=\mathbf{X} B^{*}+\mathbf{X}\left(I-B^{*}\right)=: \underline{\mathbf{Z}} A^{t}+\underline{\mathbf{E}}
$$

with $\operatorname{Cov}(A \underline{Z}, \underline{E})=B^{*} \Gamma\left(I-B^{*}\right), \operatorname{Cov}(\underline{E})=\left(I-B^{*}\right) \Gamma\left(I-B^{*}\right)$, and $\operatorname{Cov}\left(\underline{Z}_{k}, \underline{Z}_{l}\right)=C_{k l}^{*}+\gamma_{k l}$. Note that in the latent model $X_{a}=Z_{k(a)}+E_{a}$, the random variables $\underline{Z}_{k}$ and $\underline{E}_{a}$ differ from $Z_{k}$ and $E_{a}$.

Our first goal is to decompose $\widehat{\Sigma}-\widehat{\Gamma}$ in such a way that the distance $\left|\underline{\mathbf{Z}}_{: k}-\underline{\mathbf{Z}}_{: j}\right|_{2}^{2}$ becomes evident. To this end, recall that $n \widehat{\Sigma}=\mathbf{X}^{t} \mathbf{X}$ and let us define $\widetilde{\Gamma}=\frac{1}{n} \underline{\mathbf{E}}^{\mathbf{t}} \underline{\mathbf{E}}$. Hence, we have

$$
n \widehat{\Sigma}=A \underline{\mathbf{Z}}^{t} \underline{\mathbf{Z}} A^{t}+n \widetilde{\Gamma}+A\left(\underline{\mathbf{Z}}^{t} \underline{\mathbf{E}}\right)+\left(\underline{\mathbf{E}}^{t} \underline{\mathbf{Z}}\right) A^{t} .
$$

Using the fact that for any vectors $v_{1}$ and $v_{2}$ we have $\left|v_{1}-v_{2}\right|_{2}^{2}=\left|v_{1}\right|_{2}^{2}+\left|v_{2}\right|^{2}-2 v_{1}^{t} v_{2}$, we can write

$$
\left[A \underline{\mathbf{Z}}^{t} \underline{\mathbf{Z}}^{t}\right]_{a b}=\frac{1}{2}\left|\left[A \underline{\mathbf{Z}}^{t}\right]_{a:}\right|_{2}^{2}+\frac{1}{2}\left|\left[A \underline{\mathbf{Z}}^{t}\right]_{b:}\right|_{2}^{2}-\frac{1}{2}\left|\left[A \underline{\mathbf{Z}}^{t}\right]_{a:}-\left[A \underline{\mathbf{Z}}^{t}\right]_{b:}\right|_{2}^{2},
$$

for any $1 \leq a, b \leq p$. We also observe that

$$
\left[A\left(\underline{\mathbf{Z}}^{t} \underline{\mathbf{E}}\right)+\left(\underline{\mathbf{E}}^{t} \underline{\mathbf{Z}}\right) A^{t}\right]_{a b}=\left[\left(A \underline{\mathbf{Z}}^{t}\right)_{a:}-\left(A \underline{\mathbf{Z}}^{t}\right)_{b:}\right]\left[\underline{\mathbf{E}}_{b:}-\underline{\mathbf{E}}_{a:}\right]+\left[A \underline{\mathbf{Z}}^{t} \underline{\mathbf{E}}\right]_{a a}+\left[A \underline{\mathbf{Z}}^{t} \underline{\mathbf{E}}\right]_{b b} .
$$

Define the $p \times p$ matrix $W$ by

$$
W_{a b}:=n\left(\widehat{\Sigma}_{a b}-\widehat{\Gamma}_{a b}\right)-\frac{1}{2}\left|\left[A \underline{\mathbf{Z}}^{t}\right]_{a:}\right|_{2}^{2}-\frac{1}{2}\left|\left[A \underline{\mathbf{Z}}^{t}\right]_{b:}\right|_{2}^{2}-\left[A \underline{\mathbf{Z}}{ }^{t} \underline{\mathbf{E}}\right]_{a a}-\left[A \underline{\mathbf{Z}}^{t} \underline{\mathbf{E}}\right]_{b b} .
$$


Combining the four displays above we have

$$
W=W_{1}+W_{2}+W_{3}+n(\Gamma-\widehat{\Gamma})
$$

with

$$
\begin{aligned}
\left(W_{1}\right)_{a b} & :=-\frac{1}{2}\left|\left[A \underline{\mathbf{Z}}^{t}\right]_{a:}-\left[A \underline{\mathbf{Z}}^{t}\right]_{b:}\right|_{2}^{2}-n B^{*} \Gamma B^{*} \\
\left(W_{2}\right)_{a b} & :=\left[\left(A \underline{\mathbf{Z}}^{t}\right)_{a:}-\left(A \underline{\mathbf{Z}}^{t}\right)_{b:}\right]\left[\underline{\mathbf{E}}_{b:}-\underline{\mathbf{E}}_{a:}\right]-n\left[B^{*} \Gamma\left(I-B^{*}\right)+\left(I-B^{*}\right) \Gamma B^{*}\right]_{a b},
\end{aligned}
$$

and

$$
W_{3}=n \widetilde{\Gamma}-n\left(I-B^{*}\right) \Gamma\left(I-B^{*}\right)
$$

for any $1 \leq a, b \leq p$. Observe from (A.11) that $W-n(\widehat{\Sigma}-\widehat{\Gamma})$ is a sum of four matrices, two of which are of the type $1 v_{1}^{t}$, and two of the type $v_{2} 1^{t}$, for some vectors $v_{1}, v_{2} \in \mathcal{R}^{p}$. Since for any two matrices $B_{1}$ and $B_{2}$ in $\mathcal{C}$, we have $B_{1} 1=B_{2} 1=1$, it follows that

$$
\left\langle W-n(\widehat{\Sigma}-\widehat{\Gamma}), B_{1}-B_{2}\right\rangle=0 .
$$

As a consequence and using the decomposition (A.12), proving (A.9) reduces to proving

$$
\left\langle W_{1}+W_{2}+W_{3}+n(\Gamma-\widehat{\Gamma}), B^{*}-B\right\rangle>0, \text { for all } B \in \mathcal{C} \text { such that } \operatorname{supp}(B) \nsubseteq \operatorname{supp}\left(B^{*}\right)
$$

We will analyze the inner product between $B^{*}-B$ and each of the four matrices in (A.16) separately in the following lemmas. Their proofs are given after the proof of this theorem.

The matrix $W_{1}$ contains the information about the clusters, as we explain below. Note that for two variables $a$ and $b$ belonging to the same group $G_{k},\left(W_{1}\right)_{a b}=-n \gamma_{k k}$. As a consequence, $\left\langle W_{1}, B^{*}\right\rangle=-n \sum_{k} m_{k} \gamma_{k k}$. For two variables $a$ and $b$ belonging to two different groups $G_{j}$ and $G_{k}$, $\left(W_{1}\right)_{a b}=-\left|\underline{\mathbf{Z}}_{: i}-\underline{\mathbf{Z}}_{: k}\right|_{2}^{2} / 2-n \gamma_{k j}$. In the sequel, we denote by $B_{G_{j}, G_{k}}$ the submatrix $\left(B_{a b}\right)_{a \in G_{j}, b \in G_{k}}$. Since all the entries of $B$ are nonnegative, and $B 1=1$,

$$
\begin{aligned}
-\left\langle W_{1}, B\right\rangle & =\frac{1}{2} \sum_{j \neq k}\left|\underline{\mathbf{Z}}_{: j}-\underline{\mathbf{Z}}_{: k}\right|_{2}^{2}\left|B_{G_{j} G_{k}}\right|_{1}+\sum_{j, k} n \gamma_{j k}\left|B_{G_{j} G_{k}}\right|_{1}, \\
& =\frac{1}{2} \sum_{j \neq k}\left[\left|\underline{\mathbf{Z}}_{: j}-\underline{\mathbf{Z}}_{: k}\right|_{2}^{2}+2 n \gamma_{j k}\right]\left|B_{G_{j} G_{k}}\right|_{1}+n \sum_{k} \gamma_{k k}\left(m_{k}-\sum_{j: j \neq k}\left|B_{G_{j} G_{k}}\right|_{1}\right) \\
& =\frac{1}{2} \sum_{j \neq k}\left(\left|\underline{\mathbf{Z}}_{: j}-\underline{\mathbf{Z}}_{: k}\right|_{2}^{2}-n \gamma_{k k}-n \gamma_{j j}+2 n \gamma_{j k}\right)\left|B_{G_{j} G_{k}}\right|_{1}+n \sum_{k} m_{k} \gamma_{k k} .
\end{aligned}
$$

Hence, we obtain

$$
\left\langle W_{1}, B^{*}-B\right\rangle=\frac{1}{2} \sum_{j \neq k}\left[\left|\underline{\mathbf{Z}}_{: j}-\underline{\mathbf{Z}}_{: k}\right|_{2}^{2}-n \gamma_{j j}-n \gamma_{k k}+2 n \gamma_{j k}\right]\left|B_{G_{j} G_{k}}\right|_{1},
$$

Each of the random variables $\left|\underline{\mathbf{Z}}_{: j}-\underline{\mathbf{Z}}_{: k}\right|_{2}^{2}$ is a quadratic form of independent random variables. As a consequence, we can apply Hanson-Wright inequalities, of the type stated in Lemma F.1 to simultaneously control all these quantities. This leads us to Lemmas A.6 - A.9, proved in Section A.4. 
Lemma A.6. Under either Assumption 1 and Condition (A.7) or Assumption 1-bis and Condition (A.8), it holds with probability higher than $1-1 / p$, that

$$
\left\langle W_{1}, B^{*}-B\right\rangle \geq \sum_{j \neq k} n \frac{\Delta_{j k}\left(C^{*}\right)}{4}\left|B_{G_{j} G_{k}}\right|_{1}
$$

simultaneously for all matrices $B \in \mathcal{C}$.

We will analyze below the three remaining cross products.

Lemma A.7. Under Assumption 1, there exists an event of probability larger than $1-2 / p$ such that the following holds simultaneously for all $B \in \mathcal{C}$

$$
\begin{aligned}
\left|\left\langle W_{2}, B^{*}-B\right\rangle\right| \leq c_{1} L^{2} \sum_{j \neq k}\left[\sqrt{\Delta_{j k}\left(C^{*}\right)|\Gamma|_{\infty}}+\frac{|D|_{\infty}}{\sqrt{m}}\right. & \left.+|R|_{\infty}+|R|_{\infty}^{1 / 2}|D|_{\infty}^{1 / 2}\right] \\
& \times[\sqrt{n \log (p)}+\log (p)]\left|B_{G_{j} G_{k}}\right|_{1}
\end{aligned}
$$

Under Assumption 1-bis, there exists an event of probability larger than $1-2 / p$ such that the following holds simultaneously for all $B \in \mathcal{C}$

$$
\left|\left\langle W_{2}, B^{*}-B\right\rangle\right| \leq c_{1}^{\prime} M \sum_{j \neq k}\left[\sqrt{n \log (p)\left[\Delta_{k(a) k(b)}\left(C^{*}\right)+\frac{|D|_{\infty}}{m}+|R|_{\infty}\right]}+M \log (p)\right]\left|B_{G_{j} G_{k}}\right|_{1} .
$$

It remains to control the term $W_{3}$ corresponding to the empirical covariance matrix of the noise $\underline{\mathbf{E}}$. This is the main technical difficulty in this proof.

Lemma A.8. Under Assumption 1, it holds with probability higher than $1-1 / p$ that

$$
\left|\left\langle W_{3}, B^{*}-B\right\rangle\right| \leq c_{L}\|\Gamma\|_{o p}\left(\sqrt{\frac{n p}{m^{2}}}+\frac{p}{m}\right) \sum_{j \neq k}\left|B_{G_{j} G_{k}}\right|_{1},
$$

simultaneously over all matrices $B \in \mathcal{C}$. Here, $c_{L}$ is a constant that only depends on $L>0$.

Under Assumption 1-bis, it holds with probability higher than $1-1 / p$ that

$$
\left|\left\langle W_{3}, B^{*}-B\right\rangle\right| \leq c_{2} M\left[\sqrt{\frac{n p\|\Gamma\|_{o p} \log (p)}{m^{2}}}+\frac{p M \log (p)}{m}\right] \sum_{j \neq k}\left|B_{G_{j} G_{k}}\right|_{1}
$$

simultaneously over all matrices $B \in \mathcal{C}$.

Finally, we control the last term $\left\langle n(\Gamma-\widehat{\Gamma}), B^{*}-B\right\rangle$ with the next lemma.

Lemma A.9. It holds that

$$
\left|\left\langle\Gamma-\widehat{\Gamma}, B^{*}-B\right\rangle\right| \leq c_{3}\left[\frac{|D-\widehat{\Gamma}|_{V}+\|R\|_{o p}}{m}+|R|_{\infty}\right] \sum_{j \neq k}\left|B_{G_{j} G_{k}}\right|_{1}
$$

simultaneously over all matrices $B \in \mathcal{C}$. 
End of the proof of Theorem A.4.. Under Assumption 1, we combine (A.18), (A.19), (A.21) and (A.23) and the assumption $L^{4} \log (p) \leq c_{4} n$ and obtain that, with probability larger than $1-c / p$,

$$
\begin{aligned}
\frac{1}{n}\left\langle W, B^{*}-B\right\rangle \geq & \sum_{j \neq k}\left[\frac{\Delta_{j k}\left(C^{*}\right)}{4}-c_{1} L^{2} \sqrt{\Delta_{j k}\left(C^{*}\right)|\Gamma|_{\infty} \frac{\log (p)}{n}}\right. \\
& -c_{2} L^{2}\left[\frac{|D|_{\infty}}{\sqrt{m}}+|R|_{\infty}+|R|_{\infty}^{1 / 2}|D|_{\infty}^{1 / 2}\right] \sqrt{\frac{\log (p)}{n}}-c_{L}\|\Gamma\|_{o p}\left[\sqrt{\frac{p}{n m^{2}}}+\frac{p}{n m}\right] \\
& \left.-c_{3}\left[\frac{|D-\widehat{\Gamma}|_{V}+\|R\|_{o p}}{m}+|R|_{\infty}\right]\right]\left|B_{G_{j} G_{k}}\right|_{1},
\end{aligned}
$$

simultaneously for all $B \in \mathcal{C}$. Condition (A.7) enforces that, for each $(j, k)$, the term in the bracket of (A.24) is positive. Hence, with probability at least $1-c_{1} / p$, the Inequality (A.16) holds since any matrix $B \in \mathcal{C}$ whose support is not included in $\operatorname{supp}\left(B^{*}\right)$ satisfies $\left|B_{G_{j} G_{k}}\right|_{1}>0$ for some $j \neq k$.

End of the proof of Theorem A.5. Let us now assume that Assumption 1-bis holds. Combining (A.17), (A.20), (A.22) and (A.23) we obtain that, with probability larger than $1-c / p$,

$$
\begin{aligned}
\frac{1}{n}\left\langle W, B^{*}-B\right\rangle \geq & \sum_{j \neq k}\left[\frac{\Delta_{j k}\left(C^{*}\right)}{4}-c_{1} M \sqrt{\Delta_{j k}\left(C^{*}\right) \frac{\log (p)}{n}}\right. \\
& -c_{2} M \sqrt{\frac{|D|_{\infty}}{m}+|R|_{\infty}} \sqrt{\frac{\log (p)}{n}}-c_{3} M^{2} \frac{p \log (p)}{n m}-c_{4} M\|\Gamma\|_{o p}^{1 / 2} \sqrt{\frac{p \log (p)}{n m^{2}}} \\
& \left.-c_{5}\left[\frac{|D-\widehat{\Gamma}|_{V}+\|R\|_{o p}}{m}+|R|_{\infty}\right]\right]\left|B_{G_{j} G_{k}}\right|_{1},
\end{aligned}
$$

simultaneously for all $B \in \mathcal{C}$. Condition (A.8) enforces that, for each $(j, k)$, the term in the bracket of (A.25) is positive and as previously that (A.16) holds with probability at least $1-c_{3} / p$.

A.4. Proofs of the Lemmas A.6 - A.9 used in the proofs of Theorems A.4 and A.5. We recall that these theorems, and the lemmas that lead to their conclusion, are used to prove Theorem 5.3.

Proof of Lemma A.6. Fix $j \neq k \in[p]$. For $i=1, \ldots, n$, the random variables $T_{i}=\underline{\mathbf{Z}}_{i j}-\underline{\mathbf{Z}}_{i k}$ are independent and identically distributed with

$$
\mathbb{E}\left[T_{i}^{2}\right]=C_{j j}^{*}+C_{k k}^{*}-2 C_{j l}^{*}+\gamma_{k k}+\gamma_{j j}-2 \gamma_{j l}=\Delta_{j k}\left(C^{*}\right)+\Delta_{j k}(\gamma) .
$$

Let us first work under assumption 1 . We have $\left\|T_{i}\right\|_{\psi_{2}}^{2} \leq \operatorname{Var}\left(T_{i}\right) L^{2}$. Applying the Hanson-Wright inequality (F.1) to $\left|\underline{\mathbf{Z}}_{: j}-\underline{\mathbf{Z}}_{: k}\right|_{2}^{2}$, we obtain, for some constant $c>0$,

$$
\left|\underline{\mathbf{Z}}_{: j}-\underline{\mathbf{Z}}_{: k}\right|_{2}^{2}-n\left(\gamma_{j j}+\gamma_{k k}-2 \gamma_{j k}\right) \geq n \Delta_{j k}\left(C^{*}\right)-c L^{2}\left(\Delta_{j k}\left(C^{*}\right)+\Delta_{j k}(\gamma)\right)(\log (p)+\sqrt{n \log (p)}),
$$

with probability higher than $1-1 /\left(p K^{2}\right)$. We derive from $\left|\Delta_{j k}(\gamma)\right| \leq 4|R|_{\infty}+2|D|_{\infty} / m$, Condition (A.7) and the condition $L^{4} \log (p) \leq c_{1} n$ that

$$
\left|\underline{\mathbf{Z}}_{: j}-\underline{\mathbf{Z}}_{: k}\right|_{2}^{2}-n\left(\gamma_{j j}+\gamma_{k k}-2 \gamma_{j k}\right) \geq n \frac{\Delta_{j k}\left(C^{*}\right)}{2}
$$


with probability higher than $1-1 /\left(p K^{2}\right)$. Taking an union bound over all $j \neq k$ leads to (A.18).

Let us now turn to Assumption 1-bis. Since $\underline{\mathbf{Z}}_{i j}=m_{j}^{-1} \sum_{a \in G_{j}} \mathbf{X}_{i a}$, it follows that $\left|T_{i}\right| \leq 2 M$ almost surely. Then, applying Hanson-Wright inequality for Bernstein-type random variables (F.2), we derive that, for some constant $c>0$

$$
\left|\underline{\mathbf{Z}}_{: j}-\underline{\mathbf{Z}}_{: k}\right|_{2}^{2}-n\left(\gamma_{j j}+\gamma_{k k}-2 \gamma_{j k}\right) \geq n \Delta_{j k}\left(C^{*}\right)-c M^{2} \log (p)-c M \sqrt{\left(\Delta_{j k}\left(C^{*}\right)+\Delta_{j k}(\gamma)\right) n \log (p)},
$$

with probability higher than $1-1 /\left(p K^{2}\right)$. We derive from $\left|\Delta_{j k}(\gamma)\right| \leq 4|R|_{\infty}+2|D|_{\infty} / m$ and Condition (A.8) that

$$
\left|\underline{\mathbf{Z}}_{: j}-\underline{\mathbf{Z}}_{: k}\right|_{2}^{2}-n\left(\gamma_{j j}+\gamma_{k k}-2 \gamma_{j k}\right) \geq n \frac{\Delta_{j k}\left(C^{*}\right)}{2},
$$

with probability higher than $1-1 /\left(p K^{2}\right)$. Taking an union bound over all $j \neq k$ leads to the second result of the lemma.

Proof of Lemma A.7. For all $a \sim b$, one has $\left(W_{2}\right)_{a b}=0$. For all $a \nsim b$, it follows from the covariance structure of $\underline{Z}$ and $\underline{E}$ that the expectation of $\mathbb{E}\left[\left(W_{2}\right)_{a b}\right]$ is zero. We will control the deviation of $W_{2}$ from zero with the Corollary F.2 of Hanson-Wright inequality, with $u=B^{*}\left(e_{a}-e_{b}\right)$ and $v=\left(I-B^{*}\right)\left(e_{a}-e_{b}\right)$. We observe that $\operatorname{Var}\left(\underline{E}_{a}-\underline{E}_{b}\right) \leq 16|\Gamma|_{\infty}$ and

$$
\operatorname{Var}\left((A \underline{Z})_{: a}-(A \underline{Z})_{: b}\right)=\operatorname{Var}\left(\underline{Z}_{k(a)}-\underline{Z}_{k(b)}\right) \leq \Delta_{k(a) k(b)}\left(C^{*}\right)+2 \frac{|D|_{\infty}}{m}+3|R|_{\infty}
$$

Let us control $\left(W_{2}\right)_{a b}$ under Assumption 1. From the variance bounds and the Corollary F.2 of Hanson-Wright inequality, we derive that

$$
\begin{aligned}
\left|\left(W_{2}\right)_{a b}\right| & \leq c L^{2} \sqrt{\operatorname{Var}\left(\underline{E}_{a}-\underline{E}_{b}\right) \operatorname{Var}\left((A \underline{Z})_{: a}-(A \underline{Z}): b\right)}[\sqrt{n \log (p)}+\log (p)] \\
& \leq c^{\prime} L^{2}\left[\sqrt{\Delta_{k(a) k(b)}\left(C^{*}\right)|\Gamma|_{\infty}}+\frac{|D|_{\infty}}{\sqrt{m}}+|R|_{\infty}+|R|_{\infty}^{1 / 2}|D|_{\infty}^{1 / 2}\right][\sqrt{n \log (p)}+\log (p)],
\end{aligned}
$$

with probability higher than $1-4 / p^{3}$. Taking an union bound over all $(a, b)$ such that $a \nsim b$ leads to the desired result.

Turning to Assumption 1-bis, we use Lemma F.3 below, which gives

$$
\left|\left(W_{2}\right)_{a b}\right| \leq c\left[M \sqrt{n \log (p)\left[\Delta_{k(a) k(b)}\left(C^{*}\right)+\frac{|D|_{\infty}}{m}+|R|_{\infty}\right]}+M^{2} \log (p)\right]
$$

with probability higher than $1-4 / p^{3}$. The result follows again from a union bound.

Proof of Lemma A.8. By definition of $\underline{\mathbf{E}}$ and $\widetilde{\Gamma}$, one has $\widetilde{\Gamma}=\left(I-B^{*}\right) \widetilde{\Gamma}\left(I-B^{*}\right)$ and therefore $B^{*} \widetilde{\Gamma}=0$. Then, Lemma A.3 entails

$$
\left|\left\langle\widetilde{\Gamma}-\left(I-B^{*}\right) \Gamma\left(I-B^{*}\right), B^{*}-B\right\rangle\right| \leq\left\|\widetilde{\Gamma}-\left(I-B^{*}\right) \Gamma\left(I-B^{*}\right)\right\|_{o p} \frac{\sum_{j \neq k}\left|B_{G_{j} G_{k}}\right|_{1}}{m} .
$$


Let us first control the random variable $\left\|\widetilde{\Gamma}-\left(I-B^{*}\right) \Gamma\left(I-B^{*}\right)\right\|_{o p}$ under Assumption 1. We apply Lemma F.4 and obtain

$$
\left\|\widetilde{\Gamma}-\left(I-B^{*}\right) \Gamma\left(I-B^{*}\right)\right\|_{o p} \leq c_{L}\|\Gamma\|_{o p}\left[\sqrt{\frac{p}{n}}+\frac{p}{n}\right]
$$

with probability higher than $1-1 / p$.

Turning to Assumption 1-bis, we observe that the random variables $\left|E_{a}\right|$ are all bounded by $2 M$. Applying the matrix Bernstein inequality (Lemma F.5 below), we obtain

$$
\left\|\widetilde{\Gamma}-\left(I-B^{*}\right) \Gamma\left(I-B^{*}\right)\right\|_{o p} \leq c M \sqrt{\frac{p\|\Gamma\|_{o p} \log (p)}{n}}+c \frac{p M^{2} \log (p)}{n},
$$

with probability higher than $1-1 / p$. Together with (A.26) and the definition of $W_{3}$, these two deviation inequalities allows us to prove the desired results.

Proof of Lemma A.9. We first observe that

$$
\left|\left\langle\Gamma-\widehat{\Gamma}, B^{*}-B\right\rangle\right|=\left|\left\langle R+D-\widehat{D}, B^{*}-B\right\rangle\right| \leq\left|\left\langle R, B^{*}-B\right\rangle\right|+\left|\left\langle D-\widehat{D}, B^{*}-B\right\rangle\right| .
$$

According to Lemma A.3

$$
\left|\left\langle R, B^{*}-B\right\rangle\right| \leq\left[\sum_{j \neq k}\left|B_{G_{j} G_{k}}\right|_{1}\right]\left(\frac{\|R\|_{o p}}{m}+6\left|B^{*} R\right|_{\infty}\right)
$$

hence

$$
\left|\left\langle\Gamma-\widehat{\Gamma}, B^{*}-B\right\rangle\right| \leq\left(\frac{\|R\|_{o p}}{m}+6|R|_{\infty}\right) \sum_{j \neq k}\left|B_{G_{j} G_{k}}\right|_{1}+\left|\left\langle D-\widehat{D}, B^{*}-B\right\rangle\right|
$$

For the second term, we follow the same approach as for $\widetilde{\Gamma}-\Gamma$. The additional ingredient is that $\left\langle D-\widehat{D}, B^{*}-B\right\rangle=\left\langle D-\widehat{D}-\alpha I_{p}, B^{*}-B\right\rangle$ for any $\alpha \in \mathbf{R}$, since $\operatorname{tr}\left(B^{*}\right)=K=\operatorname{tr}(B)$. According to Lemma A.3, for any $\alpha \in \mathbf{R}$, the following holds:

$$
\begin{aligned}
\left|\left\langle D-\widehat{D}, B^{*}-B\right\rangle\right| & =\left|\left\langle D-\widehat{D}-\alpha I_{p}, B^{*}-B\right\rangle\right| \\
& \leq 2\left[\sum_{j \neq k}\left|B_{G_{j} G_{k}}\right|_{1}\right]\left(\frac{\left\|D-\widehat{D}-\alpha I_{p}\right\|_{o p}}{2 m}+3\left|B^{*}\left[(D-\widehat{D})-\alpha I_{p}\right]\right|_{\infty}\right) \\
& \leq \frac{7\left\|D-\widehat{D}-\alpha I_{p}\right\|_{\infty}}{m}\left[\sum_{j \neq k}\left|B_{G_{j} G_{k}}\right|_{1}\right],
\end{aligned}
$$

where we used in the last line that $\alpha I_{p}, D$ and $\widehat{D}$ are diagonal matrices.

We fix $\alpha=\left(\max _{k}\left(D_{k k}-\widehat{D}_{k k}\right)+\min _{k}\left(D_{k k}-\widehat{D}_{k k}\right)\right) / 2$. The above inequality simplifies to

$$
\left|\left\langle D-\widehat{D}, B^{*}-B\right\rangle\right| \leq \frac{7\|D-\widehat{D}\|_{V}}{2 m} \sum_{j \neq k}\left|B_{G_{j} G_{k}}\right|_{1} .
$$

The result follows. 
A.5. Guarantees for the estimator (5.7) of $\boldsymbol{\Gamma}$. Proposition A.10 controls the estimation error of estimator $\widehat{\Gamma}$ defined by (5.7) under both the exact model and the approximate block $G$-block model (1.4). We set $v^{2}=\min _{c \neq d} \operatorname{Var}\left(X_{c}-X_{d}\right)$.

Proposition A.10. Assume that $\Gamma$ either

(a) is diagonal $(R=0)$;

(b) or fulfills the diagonal dominance assumption (6.2).

Assume also that $\Delta\left(C^{*}\right) \geq 0$. Then, the two following results holds.

(i) Under Assumption 1, there exist three numerical constants $c_{1}-c_{3}$ such that when $m \geq 3$ and $L^{4} \log (p) \leq c_{1} n$, with probability larger than $1-c_{3} / p$, the estimator $\widehat{\Gamma}$ defined by (5.7) satisfies

$$
|\widehat{\Gamma}-\Gamma|_{V} \leq 2|\widehat{\Gamma}-\Gamma|_{\infty} \leq c_{2}\left(\sqrt{|R|_{\infty}|\Gamma|_{\infty}}+|\Gamma|_{\infty} L^{2} \sqrt{\frac{\log (p)}{n}}\right) .
$$

(ii) Under Assumption 1-bis, there exist three numerical constants $c_{1}-c_{3}$ such that when $m \geq 3$ and $\log (p) \leq c_{1}(v / M)^{2} n$, with probability larger than $1-c_{3} / p$, the estimator $\widehat{\Gamma}$ defined by (5.7) satisfies

$$
|\widehat{\Gamma}-\Gamma|_{V} \leq 2|\widehat{\Gamma}-\Gamma|_{\infty} \leq c_{2}\left(\sqrt{|R|_{\infty}|\Gamma|_{\infty}}+M \sqrt{\frac{|\Gamma|_{\infty} \log (p)}{n}}+M^{2} \frac{\log (p)}{n}\right)
$$

Proof of Proposition A.10. To ease the presentation of this proof, we introduce the new notation

$$
n e_{1}(a):=\underset{b \in[p] \backslash\{a\}}{\operatorname{argmin}} V(a, b) \quad \text { and } \quad n e_{2}(a):=\underset{b \in[p] \backslash\left\{a, n e_{1}(a)\right\}}{\operatorname{argmin}} V(a, b) .
$$

(i) We start with the first part of the proposition. Let $a, b_{1}, b_{2}$ be three different indices. Under Assumption 1, the Corollary F.2 of Hanson-Wright inequality gives that with probability at least $1-p^{-4}$

$$
\begin{aligned}
& \mid \frac{1}{n}\left\langle\mathbf{X}_{: a}-\mathbf{X}_{: b_{1}}, \mathbf{X}_{: a}-\mathbf{X}_{: b_{2}}\right\rangle-\operatorname{Cov}\left(X_{a}-X_{b_{1}}, X_{a}-X_{b_{2}}\right) \mid \\
& \leq c L^{2} \sqrt{\operatorname{Var}\left(X_{a}-X_{b_{1}}\right) \operatorname{Var}\left(X_{a}-X_{b_{2}}\right)}\left(\sqrt{\frac{\log (p)}{n}}+\frac{\log (p)}{n}\right) .
\end{aligned}
$$

Applying the inequality $2 a b \leq a^{2}+b^{2}$, and a union bound, we obtain that the inequalities

$$
\begin{aligned}
\mid \frac{1}{n}\left\langle\mathbf{X}_{: a}-\mathbf{X}_{: b_{1}}, \mathbf{X}_{: a}-\mathbf{X}_{: b_{2}}\right\rangle- & \operatorname{Cov}\left(X_{a}-X_{b_{1}}, X_{a}-X_{b_{2}}\right) \mid \\
& \leq c^{\prime} L^{2}\left[\operatorname{Var}\left(X_{a}-X_{b_{1}}\right)+\operatorname{Var}\left(X_{a}-X_{b_{2}}\right)\right]\left(\sqrt{\frac{\log (p)}{n}}+\frac{\log (p)}{n}\right)
\end{aligned}
$$

hold simultaneously over all triplets of different indices $a, b_{1}, b_{2}$, with probability $1-1 / p$. Decomposing these variance and covariance terms, we obtain

$$
\begin{aligned}
\operatorname{Cov}\left(X_{a}-\right. & \left.X_{b_{1}}, X_{a}-X_{b_{2}}\right) \\
& =D_{a a}+\frac{1}{2}\left(\Delta_{k(a) k\left(b_{1}\right)}\left(C^{*}\right)+\Delta_{k(a) k\left(b_{2}\right)}\left(C^{*}\right)-\Delta_{k\left(b_{2}\right) k\left(b_{1}\right)}\left(C^{*}\right)\right)+R_{b_{1} b_{2}}-R_{a b_{1}}-R_{a b_{2}},
\end{aligned}
$$


and $\operatorname{Var}\left(X_{a}-X_{b}\right)=D_{a a}+D_{b b}+\Delta_{k(a) k(b)}\left(C^{*}\right)-2 R_{a b}$. Hence

$\left|\operatorname{Cov}\left(X_{a}-X_{b_{1}}, X_{a}-X_{b_{2}}\right)-D_{a a}\right| \leq 3|R|_{\infty}+\frac{\left|\Delta_{k(a) k\left(b_{1}\right)}\left(C^{*}\right)\right|+\left|\Delta_{k(a) k\left(b_{2}\right)}\left(C^{*}\right)\right|+\left|\Delta_{k\left(b_{1}\right) k\left(b_{2}\right)}\left(C^{*}\right)\right|}{2}$.

For $i=1,2$, write $t_{i}:=\left|\Delta_{k(a) k\left(n e_{i}(a)\right)}\left(C^{*}\right)\right|$ and $t_{12}=\left|\Delta_{k\left(n e_{1}(a)\right) k\left(n e_{2}(a)\right)}\left(C^{*}\right)\right|$. Since $\log (p) \leq$ $c_{1} L^{-4} n$, the previous inequalities entail that

$$
\left|\widehat{\Gamma}_{a a}-D_{a a}\right| \leq c\left(|R|_{\infty}+\left(t_{1}+t_{2}+t_{12}\right)+L^{2}|\Gamma|_{\infty} \sqrt{\frac{\log (p)}{n}}\right)
$$

with probability at least $1-1 / p$. As a consequence, we only have to prove that $t_{1}, t_{2}$ and $t_{12}$ are smaller than $c\left(\sqrt{|R|_{\infty}|\Gamma|_{\infty}}+L^{2}|\Gamma|_{\infty} \sqrt{\log (p) / n}\right)$ with probability at least $1-c / p$. We focus on $t_{1}$, the arguments for $t_{2}$ and $t_{12}$ being similar.

First note that $t_{1}=0$ if $k(a)=k\left(n e_{1}(a)\right)$ so that we may assume henceforth that $k(a) \neq$ $k\left(n e_{1}(a)\right)$. We have the following.

Lemma A.11. Assume that $\Gamma$ either

(a) is diagonal;

(b) or fulfills the diagonal dominance assumption (6.2).

Assume also that $\Delta\left(C^{*}\right) \geq 0$. Then, there exists a numerical constant $c_{1}$, such that, outside an event of probability less than $1 / p^{2}$, we have

(i) under Assumption 1,

$$
\left|\left\langle\mathbf{X}_{: a}-\mathbf{X}_{: n e_{1}(a)}, \mathbf{X}_{: c}-\mathbf{X}_{: d}\right\rangle\right| \leq c_{1}\left(\sqrt{n|R|_{\infty}}+L^{2}|\Gamma|_{\infty}^{1 / 2} \sqrt{\log (p)}\right)\left|\mathbf{X}_{: c}-\mathbf{X}_{: d}\right|_{2},
$$

simultaneously over all $c, d \neq\left(a, n e_{1}(a)\right)$;

(ii) under Assumption 1-bis,

$$
\left|\left\langle\mathbf{X}_{: a}-\mathbf{X}_{: n e_{1}(a)}, \mathbf{X}_{: c}-\mathbf{X}_{: d}\right\rangle\right| \leq c_{1}\left(\sqrt{n|R|_{\infty}}+M \sqrt{\log (p)}\right)\left|\mathbf{X}_{: c}-\mathbf{X}_{: d}\right|_{2}
$$

simultaneously over all $c, d \neq\left(a, n e_{1}(a)\right)$.

Similar bounds also hold for ne $e_{2}(a)$ instead of ne $e_{1}(a)$.

This lemma is proved in Section A.6. Below, $c^{\prime}$ denotes a numerical constant, whose value may vary from line to line.

For any $c$ and $d$, the variance of $X_{c}-X_{d}$ is less than $\Delta_{k(c) k(d)}\left(C^{*}\right)+2|D|_{\infty}+2|R|_{\infty}$. As a consequence, Hanson-Wright inequality together with an union bound over all $c, d \in[p]$ and the condition $L^{4} \log (p) \leq c_{1} n$ leads to

$$
\left|\mathbf{X}_{: c}-\mathbf{X}_{: d}\right|_{2} \leq c^{\prime} \sqrt{n\left[4|\Gamma|_{\infty}+\Delta_{k(c) k(d)}\left(C^{*}\right)\right]},
$$

simultaneously over all $c \neq d$, with probability $1-1 / p^{2}$. Take $c$ and $d$ any two indices such that $k(a)=k(c)$ and $k\left(n e_{1}(a)\right)=k(d)$. So combined with Lemma A.11, we get with probability at least $1-2 p^{-2}$

$$
\left|\left\langle\mathbf{X}_{: a}-\mathbf{X}_{: n e_{1}(a)}, \mathbf{X}_{: c}-\mathbf{X}_{: d}\right\rangle\right| \leq c^{\prime}\left(\sqrt{n|R|_{\infty}}+L^{2}|\Gamma|_{\infty}^{1 / 2} \sqrt{\log (p)}\right) \sqrt{n\left[|\Gamma|_{\infty}+t_{1}\right]}
$$


Let us now lower bound the left hand-side of the above inequality. For any $c$ in the same group as $a$ and $b$ in the same group as $d$, we have $\mathbb{E}\left[\left\langle X_{a}-X_{b}, X_{c}-X_{d}\right\rangle\right]=\Delta_{k(b) k(a)}\left(C^{*}\right)+R_{a c}+R_{b d}-R_{b c}-R_{a b}$. Therefore, Corollary F.2 of the Hanson-Wright inequality yields, with probability at least $1-p^{4}$,

$$
\left|\left\langle\mathbf{X}_{: a}-\mathbf{X}_{: b}, \mathbf{X}_{: c}-\mathbf{X}_{: d}\right\rangle\right| \geq n \Delta_{k(b) k(a)}\left(C^{*}\right)-4 n|R|_{\infty}-c^{\prime} L^{2} \sqrt{n \log (p)}\left[\Delta_{k(b) k(a)}\left(C^{*}\right)+|\Gamma|_{\infty}\right] .
$$

As a consequence, for any $c$ and $d$ such that $k(a)=k(c)$ and $k\left(n e_{1}(a)\right)=k(d)$, we get from $L^{4} \log (p) \leq c_{1} n$ and a union bound

$$
\left|\left\langle\mathbf{X}_{: a}-\mathbf{X}_{: n e_{1}(a)}, \mathbf{X}_{: c}-\mathbf{X}_{: d}\right\rangle\right| \geq n t_{1} / 2-4 n|R|_{\infty}-c^{\prime} L^{2}|\Gamma|_{\infty} \sqrt{n \log (p)}
$$

with probability $1-1 / p^{2}$. Gathering the previous bound with (A.35), Condition (A.33), Assumption 1 and $L^{4} \log (p) \leq n$, we conclude that

$$
t_{1} \leq c^{\prime}\left[|R|_{\infty}^{1 / 2}|\Gamma|_{\infty}^{1 / 2}+L^{2}|\Gamma|_{\infty} \sqrt{\frac{\log (p)}{n}}\right]
$$

simultaneously for all $a$, with probability $1-c_{3} / p$. Together with (A.32), this concludes the proof of the first part.

Proof of (ii). We will follow the same lines of proof as for part (i), adjusting the probabilistic inequalities to the bounded setting. Under Assumption 1-bis, Lemma F.3 below ensures that, simultaneously for all triplet $\left(a, b_{1}, b_{2}\right)$ of distinct indices,

$$
\begin{aligned}
\mid \frac{1}{n}\left\langle X_{a}-X_{b_{1}}, X_{a}-X_{b_{2}}\right\rangle-\operatorname{Cov}\left(X_{a}-X_{b_{1}}, X_{a}\right. & \left.-X_{b_{2}}\right) \mid \\
& \leq c^{\prime}\left(M \sqrt{\frac{\operatorname{Var}\left(X_{a}-X_{b_{1}}\right) \log (p)}{n}}+M^{2} \frac{\log (p)}{n}\right),
\end{aligned}
$$

with probability at least $1-p^{-4}$. Plugging the estimates (A.30) and (A.31), we get with probability $1-1 / p^{2}$

$$
\begin{aligned}
\left|\widehat{\Gamma}_{a a}-D_{a a}\right| & \leq c^{\prime}\left(|R|_{\infty}+\left(t_{1}+t_{2}+t_{12}\right)+M \sqrt{|\Gamma|_{\infty}+t_{1}} \sqrt{\frac{\log (p)}{n}}+M^{2} \frac{\log (p)}{n}\right) \\
& \leq c^{\prime \prime}\left(|R|_{\infty}+\left(t_{1}+t_{2}+t_{12}\right)+M \sqrt{\frac{|\Gamma|_{\infty} \log (p)}{n}}+M^{2} \frac{\log (p)}{n}\right),
\end{aligned}
$$

where we used for the last inequality $a \sqrt{x+y} \leq y+a \sqrt{2 x}+a^{2}$ for any $a, x, y \geq 0$.

So again, as in the first part of the proof, we only need to upper bound the bias terms $t_{1}, t_{2}, t_{12}$ by $c^{\prime}\left(\sqrt{|\Gamma|_{\infty}|R|_{\infty}}+M \sqrt{|\Gamma|_{\infty} \log (p) / n}+M^{2} \log (p) / n\right)$.

For any $c$ and $d$ such that $k(a)=k(c)$ and $k\left(n e_{1}(a)\right)=k(d)$, Hanson-Wright inequality together with the condition $M^{2} \log (p) \leq c_{1} v^{2} n$ give

$$
\begin{aligned}
\frac{1}{n}\left|\mathbf{X}_{: c}-\mathbf{X}_{: d}\right|_{2}^{2} & \leq \operatorname{Var}\left(X_{c}-X_{d}\right)+c M \sqrt{\frac{\operatorname{Var}\left(X_{c}-X_{d}\right) \log (p)}{n}}+c M^{2} \frac{\log (p)}{n} \\
& \leq c^{\prime} \operatorname{Var}\left(X_{c}-X_{d}\right) \leq c^{\prime \prime}\left(|\Gamma|_{\infty}+t_{1}\right),
\end{aligned}
$$


with probability $1-1 / p^{2}$. So combined with Lemma A.11, proved below, we get with probability at least $1-2 p^{-2}$

$$
\left|\left\langle\mathbf{X}_{: a}-\mathbf{X}_{: n e_{1}(a)}, \mathbf{X}_{: c}-\mathbf{X}_{: d}\right\rangle\right| \leq c^{\prime}\left(\sqrt{n|R|_{\infty}}+M \sqrt{\log (p)}\right) \sqrt{n\left[|\Gamma|_{\infty}+t_{1}\right]} .
$$

Applying again the Lemma F.3 and $M^{2} \log (p) \leq c_{1} v^{2} n$, we obtain

$$
\begin{aligned}
\left|\left\langle\mathbf{X}_{: a}-\mathbf{X}_{: n e_{1}(a)}, \mathbf{X}_{: c}-\mathbf{X}_{: d}\right\rangle\right| & \geq n t_{1}-4 n|R|_{\infty}-c M \sqrt{n \operatorname{Var}\left(X_{c}-X_{d}\right) \log (p)}-c M^{2} \log (p) \\
& \geq n t_{1}-4 n|R|_{\infty}-c^{\prime} M \sqrt{|\Gamma|_{\infty}+t_{1}} \sqrt{n \log (p)} .
\end{aligned}
$$

with probability $1-1 / p^{2}$. Hence, as in the first part of the proof, we conclude that

$$
t_{1} \leq c^{\prime}\left[|R|_{\infty}^{1 / 2}|\Gamma|_{\infty}^{1 / 2}+M \sqrt{\frac{|\Gamma|_{\infty} \log (p)}{n}}\right]
$$

simultaneously for all $a$, with probability $1-c_{3} / p$. Together with (A.36), this concludes the proof of the second part of the proposition.

\section{A.6. Proof of Lemma A.11.}

Proof of Lemma A.11. In this proof, $c^{\prime}$ denotes a numerical constant, whose value can vary from line to line.

Since $m \geq 3$, there exists two indices $b_{1}$ and $b_{2}$ other than $a$ belonging to the group $G_{k(a)}$. Fix any $c$ and $d$ different from $a$ and $b_{1}$.

Under Assumption 1, by Hanson-Wright inequality and since $\log (p) \leq c_{1} L^{-4} n$, it holds that

$$
\left|\mathbf{X}_{: c}-\mathbf{X}_{: d}\right|_{2}^{2} \geq n \operatorname{Var}\left(X_{c}-X_{d}\right)\left(1-c^{\prime} L^{2}\left(\sqrt{\frac{\log (p)}{n}}+\frac{\log (p)}{n}\right)\right) \geq n \operatorname{Var}\left(X_{c}-X_{d}\right) / 2
$$

with probability $1-1 / p^{5}$.

Similarly, under Assumption 1-bis, by Hanson-Wright inequality and since $M^{2} \log (p) \leq c_{1} v^{2} n$, we get

$\left|\mathbf{X}_{: c}-\mathbf{X}_{: d}\right|_{2}^{2} \geq n \operatorname{Var}\left(X_{c}-X_{d}\right)-c^{\prime}\left(M \sqrt{\operatorname{Var}\left(X_{c}-X_{d}\right) n \log (p)}+M^{2} \log (p)\right) \geq n \operatorname{Var}\left(X_{c}-X_{d}\right) / 2$,

with probability $1-1 / p^{5}$.

The random variable $\left\langle\mathbf{X}_{: a}-\mathbf{X}_{: b_{i}}, \mathbf{X}_{: c}-\mathbf{X}_{: d}\right\rangle$ has expectation $n\left(R_{a c}-R_{b_{i} c}+R_{b_{i} d}-R_{a d}\right)$. Under the diagonal dominance assumption (6.2) of the main text [2] and the assumption $\Delta\left(C^{*}\right) \geq 0$, we have

$$
\begin{aligned}
\left|\mathbb{E}\left[\left\langle\mathbf{X}_{: a}-\mathbf{X}_{: b_{i}}, \mathbf{X}_{: c}-\mathbf{X}_{: d}\right\rangle\right]\right| & \leq n\left(\left|R_{a c}\right|+\left|R_{b_{i} c}\right|+\left|R_{b_{i} d}\right|+\left|R_{a d}\right|\right) \\
& \leq 2 n|R|_{\infty}^{1 / 2} \sqrt{D_{c c}+D_{d d}-2 R_{c d}} \\
& \leq 4 \sqrt{n|R|_{\infty}}\left|\mathbf{X}_{: c}-\mathbf{X}_{: d}\right|_{2} .
\end{aligned}
$$

We observe that the inequality $\left|\mathbb{E}\left[\left\langle\mathbf{X}_{: a}-\mathbf{X}_{: b_{i}}, \mathbf{X}_{: c}-\mathbf{X}_{: d}\right\rangle\right]\right| \leq 4 \sqrt{n|R|_{\infty}}\left|\mathbf{X}_{: c}-\mathbf{X}_{: d}\right|_{2}$ still holds when $\Gamma$ is diagonal (i.e. $R=0$ ), since in this case the left-hand side is equal to zero. 
Under Assumption 1, by Hanson-Wright inequality, we obtain with probability $1-1 / p^{5}$

$$
\begin{aligned}
\left|\left\langle\mathbf{X}_{: a}-\mathbf{X}_{: b_{i}}, \mathbf{X}_{: c}-\mathbf{X}_{: d}\right\rangle\right| & \leq\left|\mathbb{E}\left[\left\langle\mathbf{X}_{: a}-\mathbf{X}_{: b_{i}}, \mathbf{X}_{: c}-\mathbf{X}_{: d}\right\rangle\right]\right|+c L^{2} \sqrt{\operatorname{Var}\left(X_{c}-X_{d}\right)|\Gamma|_{\infty} \sqrt{n \log (p)}} \\
& \leq\left(4 \sqrt{n|R|_{\infty}}+c L^{2} \sqrt{2|\Gamma|_{\infty} \log (p)}\right)\left|\mathbf{X}_{: c}-\mathbf{X}_{: d}\right|_{2} .
\end{aligned}
$$

Similarly, under Assumption 1-bis, by Hanson-Wright inequality and $M^{2} \log (p) \leq c_{1} v^{2} n$, we obtain with probability $1-1 / p^{5}$

$$
\begin{aligned}
\left|\left\langle\mathbf{X}_{: a}-\mathbf{X}_{: b_{i}}, \mathbf{X}_{: c}-\mathbf{X}_{: d}\right\rangle\right| & \leq\left|\mathbb{E}\left[\left\langle\mathbf{X}_{: a}-\mathbf{X}_{: b_{i}}, \mathbf{X}_{: c}-\mathbf{X}_{: d}\right\rangle\right]\right|+c M \sqrt{\operatorname{Var}\left(X_{c}-X_{d}\right)} \sqrt{n \log (p)}+c M^{2} \log (p) \\
& \leq\left(4 \sqrt{n|R|_{\infty}}+c^{\prime} M \sqrt{\log (p)}\right)\left|\mathbf{X}_{: c}-\mathbf{X}_{: d}\right|_{2} .
\end{aligned}
$$

By the definition of $n e_{1}(a)$ and $n e_{2}(a)$ and a union bound, we obtain the desired result.

APPENDIX B: PROOF OF RESULTS CONCERNING MODEL IDENTIFIABILITY

\section{B.1. Proofs of Section 2 and 6.1.}

Proof of Proposition 2.2. We first observe that if $\mathrm{G}$ is such that $\Sigma=A C A^{t}+\Gamma$ holds, with $A$ associated to $G$, then $G$ is a sub-partition of $G^{*}$. To see this, note that for any $a, b$ belonging to the same group of the partition $G$, we have $C O D(a, b)=0$. Hence $a, b$ are in a same group of the partition $G^{*}$, therefore $G$ is a sub-partition of $G^{*}$. Furthermore, for any sub-partition $G$ of $G^{*}$, $M C O D(\Sigma, G)=0$ unless $G=G^{*}$. Thus, $G=G^{*}$.

Proof of Corollary 2.3. We start with the following lemma that proves the inequalities in display (1.8) of the main text [2].

Lemma B.1. Assume that $G$ is a partition, such that $\Sigma=A C A^{t}+\Gamma$ with $\Gamma$ diagonal and $A$ associated to $G$. Then, we have:

(a) $2 \lambda_{K}(C) \leq \Delta(C)$

(b) if the size $m$ of the smallest group of $G$ is larger than one, then

$$
\Delta(C) \leq 2 \operatorname{MCOD}(\Sigma, G)
$$

(c) if $C$ is semi-positive definite, then

$$
\operatorname{MCOD}(\Sigma, G) \leq \sqrt{\Delta(C)} \max _{k=1, \ldots, K} \sqrt{C_{k k}} .
$$

The proof of Corollary 2.3 relies on the inequality (B.1) which holds since $m \geq 2$. The result then follows from Proposition 2.2.

Proof of Lemma B.1. The first inequality holds because

$$
\Delta(C)=\min _{j<k}\left(e_{j}-e_{k}\right)^{t} C\left(e_{j}-e_{k}\right) \geq 2 \lambda_{K}(C) .
$$

Let us prove the second inequality. For any $k \neq j$, and for any pair of $a, a^{\prime} \in G_{k}$ and $b, b^{\prime} \in G_{j}$ we have

$$
C_{k k}+C_{j j}-2 C_{j k}=\Sigma_{a a^{\prime}}-\Sigma_{b a^{\prime}}+\Sigma_{b b^{\prime}}-\Sigma_{a b^{\prime}} \leq 2 C O D(a, b) .
$$


Since the inequalities are valid for any $a, b$ in distincts groups, the bound $\Delta(C) \leq 2 \operatorname{MCOD}(\Sigma, G)$ follows.

For the last bound, we observe that for any $a \in G_{k}, b \in G_{j}$ and $c \in G_{\ell}$, we have

$$
\left|\Sigma_{a c}-\Sigma_{b c}\right|=\left|C_{k \ell}-C_{j \ell}\right|=\left(e_{k}-e_{j}\right)^{t} C e_{\ell} \leq \sqrt{\left(e_{k}-e_{j}\right)^{t} C\left(e_{k}-e_{j}\right)} \sqrt{e_{\ell}^{t} C e_{\ell}},
$$

where the last inequality holds since $C$ is positive semi-definite. The last inequality of the lemma follows.

B.2. Proof of Proposition 6.1. Consider any covariance matrix $\Sigma$ that either satisfies $\rho_{1}(\Sigma, K)>$ 2 or $\rho_{2}(\Sigma, K)>8$. Thus, there either exists $(A, C, D, R) \in \mathcal{P}_{1}(\Sigma, K)$ with $\operatorname{MCOD}(\Sigma, G)>2|R|_{\infty}$, where $G$ be the partition associated to $A$; or $(A, C, D, R) \in \mathcal{P}_{2}(\Sigma, K)$ with $\Delta(C)>8|R|_{\infty}$. Let us consider another quadruplet $\left(A^{\prime}, C^{\prime}, D^{\prime}, R^{\prime}\right) \in \mathcal{P}_{1}(\Sigma, K)$ (resp. $\left.\mathcal{P}_{2}(\Sigma, K)\right)$ with $M C O D\left(\Sigma, G^{\prime}\right)>$ $2|R|_{\infty}$ (resp. $\left.\Delta\left(C^{\prime}\right)>8\left|R^{\prime}\right|_{\infty}\right)$ and with associated partition $G^{\prime}$. We prove below $G=G^{\prime}$, based on the following lemma.

Lemma B.2. Assume either that $(A, C, D, R) \in \mathcal{P}_{1}(\Sigma, K)$ with $M C O D(\Sigma, G)>2|R|_{\infty}$ or that $(A, C, D, R) \in \mathcal{P}_{2}(\Sigma, K)$ with $\Delta(C)>8|R|_{\infty}$. For any integer a between 1 and $p$ set

$$
V(a)=\left\{a^{\prime}: a^{\prime} \neq a, \operatorname{COD}\left(a, a^{\prime}\right) \leq 2|R|_{\infty}\right\} .
$$

Writing $k(a)$ for the integer such that $a \in G_{k(a)}$, we have $V(a)=G_{k(a)} \backslash\{a\}$.

Before proving this lemma, let us explain why it implies that $G=G^{\prime}$. By symmetry, we can assume that $\left|R^{\prime}\right|_{\infty} \leq|R|_{\infty}$. So $V^{\prime}(a) \subset V(a)$ and hence $G_{k^{\prime}(a)}^{\prime} \subset G_{k(a)}$. To conclude, we observe that $G^{\prime}$ is then a sub-partition of $G$, with the same number $K$ of groups, so the two partitions $G$ and $G^{\prime}$ are equal. The three assertions (i), (ii) and (iii) of the Proposition 6.1 follows.

To conclude, it remains to prove Lemma B.2.

Proof of Lemma B.2. Since $\Sigma=A C A^{t}+R+D$, writing $k(a)$ for the integer such that $a \in G_{k(a)}$, we have $\Sigma_{a b}=C_{k(a) k(b)}+R_{a b}$ for any $a \neq b$.

First, if $a^{\prime} \in G_{k(a)} \backslash\{a\}$, then $k\left(a^{\prime}\right)=k(a)$, so

$$
\left|\Sigma_{a c}-\Sigma_{a^{\prime} c}\right|=\left|R_{a c}+C_{k(a) k(c)}-C_{k(a) k(c)}-R_{a^{\prime} c}\right| \leq 2|R|_{\infty}
$$

for any $c \neq a, a^{\prime}$. So $a^{\prime} \in V(a)$ and hence $G_{k(a)} \backslash\{a\} \subset V(a)$.

Conversely, let us prove that $V(a) \subset G_{k(a)} \backslash\{a\}$. If $(A, C, D, R)$ belongs to $\mathcal{P}_{1}(\Sigma, K)$, then any two $a$ and $b$ in different groups satisfy $C O D(a, b)>2|R|_{\infty}$, implying that $V(a) \subset G_{k(a)} \backslash\{a\}$. Now assume that $(A, C, D, R)$ belongs to $\mathcal{P}_{2}(\Sigma, K)$ and that there exists $a^{\prime} \in V(a)$ such that $k\left(a^{\prime}\right) \neq k(a)$. Hence, $m>1$ and $a^{\prime} \notin G_{k(a)}$. We can therefore find $b \in G_{k(a)} \backslash\{a\}$ and $b^{\prime} \in G_{k\left(a^{\prime}\right)} \backslash\left\{a^{\prime}\right\}$. We have

$$
\begin{gathered}
C_{k(a) k(a)}-C_{k(a) k\left(a^{\prime}\right)}=\Sigma_{a b}-\Sigma_{a^{\prime} b}-R_{a b}+R_{a^{\prime} b} \\
C_{k(a) k\left(a^{\prime}\right)}-C_{k\left(a^{\prime}\right) k\left(a^{\prime}\right)}=\Sigma_{a b^{\prime}}-\Sigma_{a^{\prime} b^{\prime}}-R_{a b^{\prime}}+R_{a^{\prime} b^{\prime}} .
\end{gathered}
$$

Since $a^{\prime} \in V(a)$, we have $C O D\left(a, a^{\prime}\right) \leq 2|R|_{\infty}$ so

$\Delta(C) \leq C_{k(a) k(a)}+C_{k\left(a^{\prime}\right) k\left(a^{\prime}\right)}-2 C_{k(a) k\left(a^{\prime}\right)}=\Sigma_{a b}-\Sigma_{a^{\prime} b}-\Sigma_{a b^{\prime}}+\Sigma_{a^{\prime} b^{\prime}}+R_{a^{\prime} b}-R_{a b}-R_{a^{\prime} b^{\prime}}+R_{a b^{\prime}} \leq 8|R|_{\infty}$,

which is in contradiction with $\Delta(C)>8|R|_{\infty}$. So it cannot hold that $k\left(a^{\prime}\right) \neq k(a)$, which means that any $a^{\prime} \in V(a)$ belongs to $G_{k(a)} \backslash\{a\}$, i.e. $V(a) \subset G_{k(a)} \backslash\{a\}$. This conclude the proof of the equality $V(a)=G_{k(a)} \backslash\{a\}$. 
B.3. Examples of $\boldsymbol{\Sigma}$ with $\boldsymbol{\rho}(\boldsymbol{\Sigma}, \boldsymbol{K})=\mathbf{8}$. Consider the matrix $\boldsymbol{\Sigma}=\left[\begin{array}{cccc}3 r & r & 0 & 0 \\ r & 3 r & 0 & 0 \\ 0 & 0 & 3 r & r \\ 0 & 0 & r & 3 r\end{array}\right]+I_{p}$ with $r>0$ and the convention that each entry in the matrix corresponds to a block of size 2 . Fixing $K=3$, we have the following decompositions for $\Sigma$

$$
\Sigma-I_{p}=\underbrace{\left[\begin{array}{cccc}
4 r & 0 & -r & -r \\
0 & 4 r & -r & -r \\
-r & -r & 2 r & 2 r \\
-r & -r & 2 r & 2 r
\end{array}\right]}_{=A_{1} C_{1} A_{1}^{t}}+\underbrace{\left[\begin{array}{cccc}
r & -r & r & r \\
-r & r & r & r \\
r & r & r & -r \\
0 & 0 & -r & r
\end{array}\right]}_{=R_{1}+D_{1}-I_{p}}=\underbrace{\left[\begin{array}{cccc}
2 r & 2 r & -r & -r \\
2 r & 2 r & -r & -r \\
-r & -r & 4 r & 0 \\
-r & -r & 0 & 4 r
\end{array}\right]}_{=A_{2} C_{2} A_{2}^{t}}+\underbrace{\left[\begin{array}{cccc}
r & -r & r & r \\
-r & r & r & r \\
r & r & -r & r \\
r & r & r & -r
\end{array}\right]}_{=R_{2}+D_{2}-I_{p}}
$$

For both decompositions, we have $\Delta\left(C_{1}\right)=\Delta\left(C_{2}\right)=8 r=8\left|R_{1}\right|_{\infty}=8\left|R_{2}\right|_{\infty}$, so that two different partitions $G_{1}$ and $G_{2}$ lead to the same ratio $\Delta\left(C_{i}\right) /\left|R_{i}\right|_{\infty}=8$. It remains to prove that $\max _{C, R} \Delta(C) /|R|_{\infty}=8$ to conclude. For any decomposition associated to size 3 partition $G$, we have $|R|_{\infty} \geq r$. By symmetry we may assume that one group of $G_{1} \cap G_{1}^{*} \neq \emptyset$ and $G_{3} \cap G_{3}^{*} \neq \emptyset$ and $G_{3} \cap G_{4}^{*} \neq \emptyset$. As consequence, $C_{3,3} \leq r+|R|_{\infty}, C_{1,1} \leq 3 r+|R|_{\infty}$ and $C_{1,3} \geq-r-|R|_{\infty}$ implying that $\Delta(C) \leq 4 r+4|R|_{\infty}$. Since $|R|_{\infty} \geq r$, we obtain $\rho_{2}(\Sigma, 3) \leq 8$. So we have prove that $\rho_{2}(\Sigma, 3)=8$ and that the maximum is achieved for at least 2 different partitions.

\section{APPENDIX C: PROOFS FOR SECTION 3: MINIMAX LOWER BOUNDS}

These proofs of Theorems 3.2 and 3.1 are based on a version of Fano's lemma that is appropriate for our application. Specifically, we will employ Birgé's Lemma (Corollary 2.18 in [7]), which we state below, translated to our problem. We let $\mathcal{S}$ denote generically either $\mathcal{M}(\eta, m)$ or $\mathcal{D}(\tau, m)$. Then:

Lemma C.1. For any partition estimator $\widehat{G}$, and for any collection of distinct covariance matrices $\Sigma^{(j)} \in \mathcal{S}, 1 \leq j \leq M$, we have

$$
\begin{aligned}
\sup _{\Sigma \in \mathcal{S}} \mathbb{P}_{\Sigma}\left(\widehat{G} \neq G^{*}\right) & \geq \max _{j=1, \ldots, M} \mathbb{P}_{\Sigma^{(j)}}\left(\widehat{G} \neq G^{(j)}\right) \\
& \geq \frac{1}{2 e+1} \wedge\left(1-\max _{j \geq 2} \frac{\mathcal{K}\left(\Sigma^{(j)}, \Sigma^{(1)}\right)}{\log (M)}\right),
\end{aligned}
$$

where $\mathcal{K}\left(\Sigma^{(j)}, \Sigma^{(1)}\right)$ denotes the Kulback-Leibler divergence between two Gaussian likelihoods based on $n$ observations.

\section{C.1. Minimax lower bounds with respect to the MCOD metric: Proof of Theorem} 3.1 . For $m=p / 2$, we observe that $\Delta\left(C^{*}\right) / 2 \leq \operatorname{MCOD}(\Sigma) \leq \Delta\left(C^{*}\right)$, so the result follows from Theorem 3.2.

For $m \leq p / 3$, we derive the lower bound by constructing an example that corresponds to partitions with $K=3$ blocks of size $p / 3$ each, and thus the size of the smallest cluster $m=p / 3$. We would expect that in this case the clusters will be easier to separate, and that $m$ will play a role in the minimax bound, however our result below shows that the perfect recovery separation rate is $O(\sqrt{\log p / n})$. To construct the class of covariance matrices to which we will apply Lemma C.1 we let $0<\epsilon<1$ and define

$$
C(\epsilon)=\left[\begin{array}{ccc}
\epsilon & \epsilon-\epsilon^{2} & -\epsilon \\
\epsilon-\epsilon^{2} & \epsilon & \epsilon \\
-\epsilon & \epsilon & 2
\end{array}\right]
$$


which is positive semi-definite. Consider the following covariance matrix with $K=3$ blocks of equal size $m=p / 3$ given by $\Sigma:=A C(\epsilon) A^{t}+I$, where $A$ is a $p \times 3$ hard assignment matrix given by $A_{j 1}=1$, if $1 \leq j \leq m$, and zero otherwise, $A_{j 2}=1$, if $m+1 \leq j \leq 2 m$, and zero otherwise and $A_{j 3}=1$, if $2 m+1 \leq j \leq 3 m$, and zero otherwise. We observe that $\operatorname{MCOD}(\Sigma)=2 \epsilon$ and $|\Sigma|_{\infty}=3$. For $a \in\{1, \ldots, m\}$ and $b \in\{m+1, \ldots, 2 m+1\}$, we construct $\Sigma^{(a, b)}$ by permuting the indices $a$ and $b$. Naturally, the candidate matrices will still correspond to the same $K=3$ and $m=p / 3$, but the groups in the corresponding partitions will have switched labels. We collect the $M=m^{2}+1$ candidate matrices in a set $\mathcal{F}$. Let $G^{\prime}$ be the partition associated with one of the generic matrices $\Sigma^{\prime} \in \mathcal{F}$. We show below that if

$$
\epsilon \leq \sqrt{\frac{0.8 \log (p / 3)}{n}} \text { and } \eta \leq 0.8 \epsilon
$$

then

$$
\begin{aligned}
& \inf _{\widehat{G}} \sup _{\Sigma \in \mathcal{M}(\eta)} \mathbb{P}_{\Sigma}(\widehat{G} \neq G) \geq \inf _{\widehat{G}} \max _{\Sigma^{\prime} \in \mathcal{F}} \mathbb{P}_{\Sigma^{\prime}}\left(\widehat{G} \neq G^{\prime}\right) \\
& \geq \frac{1}{2 e+1} \text {. }
\end{aligned}
$$

To apply Lemma C.1, we calculate below the KL-divergence $\mathcal{K}\left(\Sigma^{(a, b)}, \Sigma\right)$. By symmetry, we can assume that $a=1$ and $b=m+1$. We write henceforth $Q$ for the permutation matrix associated to the transposition of 1 and $m+1$ and $\Sigma^{\prime}=Q \Sigma Q^{t}$. We start by writing out the eigenvalues and the corresponding eigenvectors of $C(\epsilon)$ :

- $\lambda_{1}=\epsilon(2-\epsilon)$ with $u_{1}^{t}=\frac{1}{\sqrt{2}}\left[\begin{array}{lll}1 & 1 & 0\end{array}\right]$,

- $\lambda_{2}=2+\epsilon^{2}$ with $u_{2}^{t}=\frac{\epsilon}{2 \sqrt{1+\epsilon^{2} / 2}}\left[\begin{array}{lll}-1 & 1 & 2 / \epsilon\end{array}\right]$,

- $\lambda_{3}=0$ with $u_{3}^{t}=\frac{1}{\sqrt{2+\epsilon^{2}}}\left[\begin{array}{lll}1 & -1 & \epsilon\end{array}\right]$.

Let $v_{k}^{t}=\frac{1}{\sqrt{m}}\left(A u_{k}\right)^{t}$, for $k=1,2$. Then, with $I$ denoting the $p \times p$ identity matrix, we have

$$
\begin{aligned}
\Sigma & =\sum_{k=1}^{2} m \lambda_{k} v_{k} v_{k}^{t}+I, \quad \Sigma^{-1}=-\sum_{k=1}^{2} \frac{m \lambda_{k}}{1+m \lambda_{k}} v_{k} v_{k}^{t}+I \\
\Sigma^{\prime}-\Sigma & =\sum_{k=1}^{2} m \lambda_{k}\left[Q v_{k} v_{k}^{t} Q^{t}-v_{k} v_{k}^{t}\right] .
\end{aligned}
$$

We have $Q v_{k}=v_{k}+\Delta_{k}$ with $\Delta_{k}^{t}=\frac{1}{\sqrt{m}}\left[\left(u_{k}\right)_{2}-\left(u_{k}\right)_{1}, 0 \ldots 0,\left(u_{k}\right)_{1}-\left(u_{k}\right)_{2}, 0 \ldots 0\right]$. Then, $\Delta_{1}=0$, $\Delta_{2}^{t}=\frac{\epsilon}{\sqrt{1+\epsilon^{2} / 2} \sqrt{m}}[1,0 \ldots 0,-1,0 \ldots 0]$, and

$$
\Sigma^{\prime}-\Sigma=m \lambda_{2}\left(v_{2} \Delta_{2}^{t}+\Delta_{2} v_{2}^{t}+\Delta_{2} \Delta_{2}^{t}\right)
$$

We notice that $v_{1} v_{1}^{t} \Delta_{2}=0$ and $v_{1} v_{1}^{t} v_{2}=0$, so putting pieces together:

$$
\Sigma^{-1} \Sigma^{\prime}=I+\Sigma^{-1}\left(\Sigma^{\prime}-\Sigma\right)=I+m \lambda_{2} F,
$$

where

$$
F:=\left(I-\frac{m \lambda_{2}}{1+m \lambda_{2}} v_{2} v_{2}^{t}\right)\left(v_{2} \Delta_{2}^{t}+\Delta_{2} v_{2}^{t}+\Delta_{2} \Delta_{2}^{t}\right)
$$


Writing $s:=\Delta_{2}^{t} v_{2}, \gamma:=\Delta_{2}^{t} \Delta_{2}$ and $\rho:=m \lambda_{2} /\left(1+m \lambda_{2}\right)$, we have

$$
\begin{aligned}
F & =\left[v_{2} \Delta_{2}^{t}+\Delta_{2} v_{2}^{t}+\Delta_{2} \Delta_{2}^{t}-\rho\left(v_{2} \Delta_{2}^{t}+s v_{2} v_{2}^{t}+s v_{2} \Delta_{2}^{t}\right)\right] \\
& =v_{2} \Delta_{2}^{t}(1-\rho(1+s))+\Delta_{2} v_{2}^{t}+\Delta_{2} \Delta_{2}^{t}-\rho s v_{2} v_{2}^{t} .
\end{aligned}
$$

Let us compute the eigenvalues of $F$. We observe that the range of $F$ is spanned by $v_{2}$ and $\Delta_{2}$, so we seek for eigenvectors $\omega=v_{2}+\alpha \Delta_{2}$. Since

$$
s=-\frac{2 \epsilon^{2}}{m\left(2+\epsilon^{2}\right)}=-\frac{2 \epsilon^{2}}{m \lambda_{2}}, \quad \text { and } \quad \gamma=\frac{4 \epsilon^{2}}{m \lambda_{2}}=-2 s,
$$

computing $F \omega$, we obtain

$$
\begin{aligned}
F \omega & =[(1-\rho(1+s))(s+\alpha \gamma)-\rho s(1+\alpha s)] v_{2}+[1+\alpha s+s+\alpha \gamma] \Delta_{2} \\
& =[(1-\rho(1+s))(1-2 \alpha)-\rho(1+\alpha s)] s v_{2}+[1+(1-\alpha) s] \Delta_{2},
\end{aligned}
$$

so

$$
\begin{aligned}
F \omega=\mu \omega & \Longleftrightarrow\left\{\begin{array}{l}
1+(1-\alpha) s=\alpha \mu \\
{[(1-\rho(1+s))(1-2 \alpha)-\rho(1+\alpha s)] s=\mu}
\end{array}\right. \\
& \Longleftrightarrow\left\{\begin{array}{l}
\alpha=\frac{1+s}{\mu+s} \\
0=\mu^{2}+\mu[\rho s(2+s)]+(1-\rho)(s+2) s .
\end{array}\right.
\end{aligned}
$$

Therefore, the two non-zero eigenvalues $\mu_{1}$ and $\mu_{2}$ of $F$ fulfill

$$
\left\{\begin{array}{l}
\mu_{1}+\mu_{2}=-\rho s(2+s) \\
\mu_{1} \mu_{2}=(1-\rho) s(2+s) .
\end{array}\right.
$$

Since $\Sigma^{-1} \Sigma^{\prime}=I+m \lambda_{2} F$, with $F$ of rank 2 , we can now compute $\mathcal{K}\left(\Sigma^{\prime}, \Sigma\right)$ :

$$
\begin{aligned}
\frac{2}{n} \mathcal{K}\left(\Sigma^{\prime}, \Sigma\right) & =\operatorname{Tr}\left(m \lambda_{2} F\right)-\log \operatorname{det}\left(I+m \lambda_{2} F\right) \\
& =m \lambda_{2}\left(\mu_{1}+\mu_{2}\right)-\log \left(1+m \lambda_{2}\left(\mu_{1}+\mu_{2}\right)+\left(m \lambda_{2}\right)^{2} \mu_{1} \mu_{2}\right) \\
& =-m \lambda_{2} \rho s(2+s)-\log \left(1-m \lambda_{2} \rho s(2+s)+\left(m \lambda_{2}\right)^{2}(1-\rho) s(2+s)\right) .
\end{aligned}
$$

We observe that $m \lambda_{2}(1-\rho)=\rho$, so the terms in the log cancel and finally, plugging the values in (C.2) into the above display, we obtain

$$
\mathcal{K}\left(\Sigma^{\prime}, \Sigma\right)=-\frac{n}{2} m \lambda_{2} \rho s(2+s)=2 n \rho \epsilon^{2}\left(1-\frac{\epsilon^{2}}{m\left(2+\epsilon^{2}\right)}\right) \leq 2 n \epsilon^{2} .
$$

Since we have $M \geq(p / 3)^{2}$ candidate matrices, Lemma C.1 ensures that when $\epsilon \leq \sqrt{\frac{2 e \log (p / 3)}{(2 e+1) n}}$ the probability of mis-clustering is larger than $1 /(2 e+1) \geq 1 / 7$. This completes the proof.

C.2. Minimax cluster lower bounds with respect to the $\Delta\left(C^{*}\right)$-metric: Proof of the first result in Theorem 3.2. It is sufficient to consider the model corresponding to $C^{*}=\tau I_{K}$ and $\Gamma=I_{p}$, so that, given a partition $G$, the covariance matrix decomposes as

$$
\Sigma_{G}=A_{G}\left(\tau I_{K}\right) A_{G}^{t}+I_{p}
$$

where $A_{G}$ is the assignment matrix associated to the partition $G$. Note that, in this case $\Delta\left(C^{*}\right)=2 \tau$ and $|\Gamma|_{\infty}=1$. Define $\mathcal{G}$ the class of all partitions of $\{1, \ldots, p\}$ into $K$ groups of identical size $m=p / K$. Recall that, as before, $\mathbb{P}_{\Sigma_{G}}$ refers to the normal distribution with covariance $\Sigma_{G}$. We will once again use Lemma C.1. To this end, we construct the candidate matrices below. 
Construction of the covariance matrices $\Sigma^{(j)}$. Let $A^{(0)}$ be the assignment matrix such that the $m$ first variables belong to the first group, the next $m$ belong to the second group and so on. In other words,

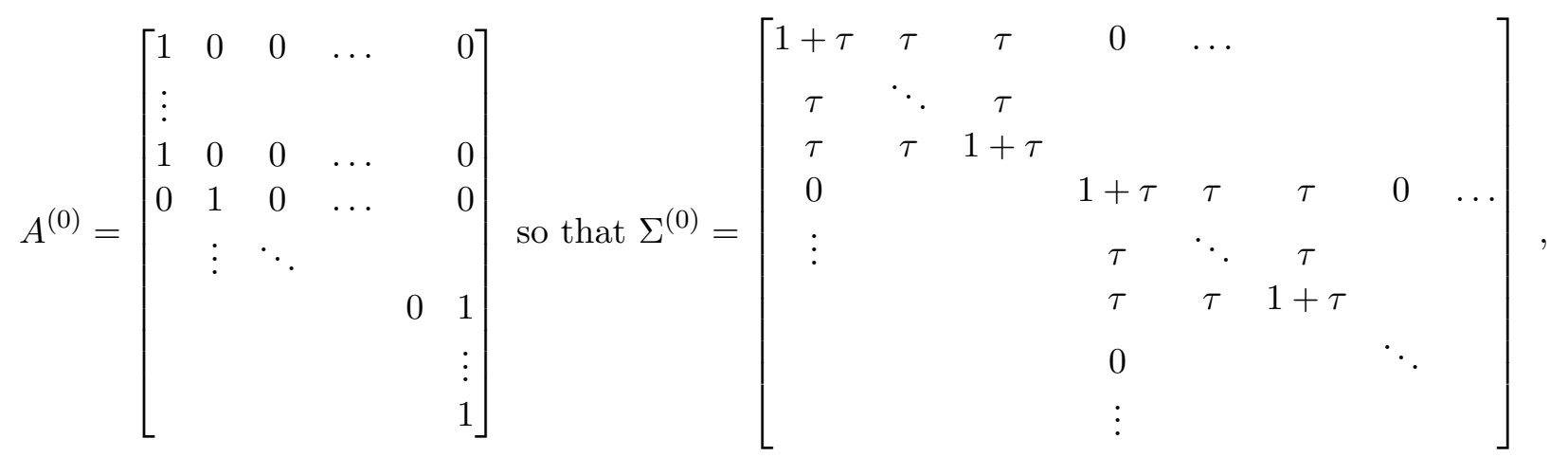

where $\Sigma^{(0)}=A^{(0)} \tau I_{K} A^{(0)^{t}}+I_{p}$. Note that the associated partition for $G^{(0)}$ is $\{\{1 \ldots m\} \ldots\{p-m+$ $1 \ldots p\}\}$. For any $a=m+1, \ldots, p$, denote $\mu_{a}$ the transposition between 1 and $a$ in $\{1 \ldots p\}$. Then, for any $a=m+1, \ldots, p$, define the assignement matrix $A^{(a)}$ and $\Sigma^{(a)}$ by

$$
A_{i j}^{(a)}=A_{\mu_{a}(i), j}^{(0)}, \quad \Sigma_{i j}^{(a)}=\Sigma_{\mu_{a}(i), \mu_{a}(j)}^{(0)} .
$$

In other words, the corresponding partition $G^{(a)}$ is obtained from $G^{(0)}$ by exchanging the role of the first and the $a$-th node.

Define the set $T:=\{0, m+1, m+2, \ldots, p\}$. Then, according to Lemma C.1, we have

$$
\inf _{\hat{G}} \max _{j \in T} \mathbb{P}_{j}\left(\hat{G} \neq G_{j}\right) \geq \frac{1}{2 e+1} \bigwedge\left(1-\frac{\sum_{j \in T \backslash\{0\}} \mathcal{K}\left(\Sigma^{(j)}, \Sigma^{(0)}\right)}{(|T|-1) \log (|T|)}\right),
$$

where, as in the previous proof, $\mathcal{K}\left(\Sigma^{(j)}, \Sigma^{(0)}\right)$ refers to the KL-divergence between two Gaussian likelihoods based on $n$ observations. By symmetry, all the Kullback divergences are equal. Since $2 e /(2 e+1) \geq 0.8$ and $1 /(2 e+1) \geq 1 / 7$, the RHS of the above inequality is further larger than $1 / 7$, provide that we show that

$$
n \mathrm{KL}\left(\mathbb{P}_{\Sigma^{(m+1)}}, \mathbb{P}_{\Sigma^{(0)}}\right) \leq 0.8 \log (p-m+1),
$$

where, $\mathrm{KL}\left(\mathbb{P}_{\Sigma^{(m+1)}}, \mathbb{P}_{\Sigma^{(0)}}\right)$ is the KL-divergence between two Gaussian distributions $(\mathrm{n}=1)$, and is evaluated below.

Lemma C.2. For any $\tau>1$ and any integers $p$ and $m$, we have

$$
\mathrm{KL}\left(\mathbb{P}_{\Sigma^{(m+1)}}, \mathbb{P}_{\Sigma^{(0)}}\right)=\frac{2(m-1) \tau^{2}}{1+m \tau}
$$

As a consequence, the desired result

$$
\inf _{\widehat{G}} \sup _{\Sigma \in \mathcal{D}(\tau)} \mathbb{P}_{\Sigma}\left[\widehat{G} \neq G^{*}\right] \geq \frac{1}{7},
$$

holds as soon as

$$
\frac{2 n(m-1) \tau^{2}}{1+m \tau} \leq 0.8 \log (p-m+1) .
$$


This last condition is satisfied as soon as

$$
\tau \leq c\left[\sqrt{\frac{\log (p)}{n(m-1)}} \bigvee \frac{\log (p)}{n}\right],
$$

for some numerical constant $c>0$. It remains to prove Lemma C.2, and we do so below.

Proof of Lemma C.2. The Kullback-Leibler divergence between two centered normal distributions writes as

$$
\mathrm{KL}\left(\mathbb{P}_{\Sigma^{(m+1)}}, \mathbb{P}_{\Sigma^{(0)}}\right)=\frac{1}{2}\left[-\log \operatorname{det}\left(\left(\Sigma^{(0)}\right)^{-1} \Sigma^{(m+1)}\right)+\operatorname{trace}\left(\left(\Sigma^{(0)}\right)^{-1} \Sigma^{(m+1)}-I_{p}\right)\right],
$$

so that we only have to compute the determinant and the trace of $A:=\left(\Sigma^{(1)}\right)^{-1} \Sigma^{(m+1)}$. We shall see that see that $A$ is a rank 2 perturbation of the identity matrix, so that we will only need to compute its two eigenvalues different from zero.

Observe that for $i=0, m+1$, the matrices $A^{(i)} A^{(i)^{t}}$ admit exactly $K$ non-zero eigenvalues that are all equal to $m$. As a consequence, we can decompose $A^{(i)} A^{(i)^{t}}=m \sum_{k=1}^{K} u_{k}^{(i)}\left(u_{k}^{(i)}\right)^{t}$ where $u_{k}^{(i)}$ is a unit vector whose non zero components are all equal to $1 / m$ and correspond to the $k$-th group in $G^{(i)}$. Note that $u_{k}^{(0)}=u_{k}^{(m+1)}$ for $k=3, \ldots, K$ as $A^{(0)}$ and $A^{(m+1)}$ only differ by rows 1 and $m+1$. The orthogonal projector $P_{i}=\sum_{k=1}^{K} u_{k}^{(i)}\left(u_{k}^{(i)}\right)^{t}$ satisfies

$$
\Sigma^{(i)}=m \tau P_{i}+I_{p}=(1+m \tau) P_{i}+\left(I_{p}-P_{i}\right) .
$$

Since $P_{i}$ and $I_{p}-P_{i}$ are orthogonal,

$$
\left(\Sigma^{(i)}\right)^{-1}=(1+m \tau)^{-1} P_{i}+\left(I_{p}-P_{i}\right)=I_{p}-\frac{m \tau}{1+m \tau} P_{i}
$$

As a consequence of the above observations, we have

$$
\begin{aligned}
A & =I_{p}+\left(\Sigma^{(0)}\right)^{-1}\left[\Sigma^{(m+1)}-\Sigma^{(0)}\right] \\
& =I_{p}+m \tau\left(P_{m+1}-P_{0}\right)-\frac{m^{2} \tau^{2}}{1+m \tau} P_{0}\left(P_{m+1}-P_{0}\right)=: I_{p}+B
\end{aligned}
$$

The matrices $P_{0}$ and $P_{m+1}$ are $k-1$ block diagonal with a first block of size $2 m \times 2 m$. Besides, $P_{0}$ and $P_{m+1}$ take the same values on all the $K-2$ remaining blocks. To compute the non-zero eigenvalues of $B$, we only to consider the restrictions $\bar{P}_{0}$ and $\bar{P}_{m+1}$ of $P_{0}$ and $P_{m+1}$ to the first $2 m \times 2 m$ entries. Also observe that the matrices $\bar{P}_{0}$ and $\bar{P}_{m+1}$ are $4 \times 4$ block-constant, with block size

$$
\left[\begin{array}{cccc}
1 \times 1 & 1 \times(m-1) & 1 \times 1 & 1 \times(m-1) \\
(m-1) \times 1 & (m-1) \times(m-1) & (m-1) \times 1 & (m-1) \times(m-1) \\
1 \times 1 & 1 \times(m-1) & 1 \times 1 & 1 \times(m-1) \\
(m-1) \times 1 & (m-1) \times(m-1) & (m-1) \times 1 & (m-1) \times(m-1)
\end{array}\right]
$$

and the entries are

$$
m \bar{P}_{0}=\left[\begin{array}{llll}
1 & 1 & 0 & 0 \\
1 & 1 & 0 & 0 \\
0 & 0 & 1 & 1 \\
0 & 0 & 1 & 1
\end{array}\right] \text { and } m \bar{P}_{m+1}=\left[\begin{array}{cccc}
1 & 0 & 0 & 1 \\
0 & 1 & 1 & 0 \\
0 & 1 & 1 & 0 \\
1 & 0 & 0 & 1
\end{array}\right]
$$

As a consequence, the non zero eigenvalues of $B$ are the same as those of

$$
C:=m \tau\left(\underline{P}_{m+1}-\underline{P}_{0}\right)-\frac{m^{2} \tau^{2}}{1+m \tau} \underline{P}_{0}\left(\underline{P}_{m+1}-\underline{P}_{0}\right)
$$


where $\underline{P}_{m+1}$ and $\underline{P}_{0}$ are two $4 \times 4$ matrices

$$
m \underline{P}_{0}=\left[\begin{array}{cccc}
1 & (m-1) & 0 & 0 \\
1 & (m-1) & 0 & 0 \\
0 & 0 & 1 & (m-1) \\
0 & 0 & 1 & (m-1)
\end{array}\right] \text { and } m \underline{P}_{m+1}=\left[\begin{array}{cccc}
1 & 0 & 0 & (m-1) \\
0 & (m-1) & 1 & 0 \\
0 & (m-1) & 1 & 0 \\
1 & 0 & 0 & (m-1)
\end{array}\right]
$$

Working out the product of matrices, we get

$$
C=-\tau\left[\begin{array}{cccc}
0 & (m-1) & 0 & -(m-1) \\
1 & 0 & -1 & 0 \\
0 & -(m-1) & 0 & (m-1) \\
-1 & 0 & 1 & 0
\end{array}\right]+\frac{(m-1) \tau^{2}}{1+m \tau}\left[\begin{array}{cccc}
1 & 1 & -1 & -1 \\
1 & 1 & -1 & -1 \\
-1 & -1 & 1 & 1 \\
-1 & -1 & 1 & 1
\end{array}\right]
$$

We observe that these two matrices have their first (resp. second) and third (resp. fourth) lines and columns opposite to each other. As a consequence, the two non-zero eigenvalues of $C^{*}$ are the same as those of

$$
\begin{aligned}
D & :=-2 \tau\left[\begin{array}{cc}
0 & (m-1) \\
1 & 0
\end{array}\right]+\frac{2(m-1) \tau^{2}}{1+m \tau}\left[\begin{array}{ll}
1 & 1 \\
1 & 1
\end{array}\right] \\
& =\frac{2 \tau}{1+m \tau}\left[\begin{array}{cc}
(m-1) \tau & -(m-1)[1+(m-1) \tau \\
-(1+\tau) & (m-1) \tau
\end{array}\right] .
\end{aligned}
$$

Straightforward computations then lead to

$$
\operatorname{tr}(D)=\frac{4(m-1) \tau^{2}}{1+m \tau}, \quad \operatorname{det}(D)=-\operatorname{tr}(D)
$$

Coming back to (C.6), we have

$$
\begin{aligned}
2 \mathrm{KL}\left(\mathbb{P}_{\Sigma^{(m+1)}}, \mathbb{P}_{\Sigma^{(0)}}\right) & \left.=-\log \operatorname{det}(A)+\operatorname{trace}\left(A-I_{p}\right)\right] \\
& =-\log \operatorname{det}(I+D)+\operatorname{tr}(D) \\
& =\operatorname{tr}(D)-\log [1+\operatorname{tr}(D)+\operatorname{det}(D)] \\
& =\frac{4(m-1) \tau^{2}}{1+m \tau} .
\end{aligned}
$$

C.3. Minimax cluster upper bounds with respect to the $\Delta\left(C^{*}\right)$-metric: Proof of the second result in Theorem 3.2. In this subsection, we introduce a variant of PECOK for achieving the optimal cluster separation threshold of Theorem 3.2 to the price of a worse computational complexity.

Without loss of generality, we assume that the number $K$ of groups divides $p$. Let $\mathcal{O}_{b} \subset \mathcal{O}$ be the collection of partnership matrices associated to balanced partitions with $K$ groups of size $m^{*}=p / K$.

Consider $\widehat{B}_{n p}=\operatorname{argmax}_{B \in \mathcal{O}_{b}}(\langle\widehat{\Sigma}-\widehat{\Gamma}, B\rangle)$ with $\widehat{\Gamma}$ given by (5.7) and $\widehat{G}_{n p}$ the associated partition. In contrast to PECOK, $\widehat{B}_{n p}$ is optimized on the non convex collection of balanced partitions $\mathcal{O}_{b}$ and we are not aware of polynomial time algorithms for computing it.

Proposition C.3. There exist $c_{1}, c_{2}, c_{3}$ three positive constants such that the following holds. If $X$ follows a Gaussian distribution, $B^{*}$ is associated to a balanced partition, $\log (p) \leq c_{2} n$ and

$$
\Delta\left(C^{*}\right) \geq c\|\Gamma\|_{\infty}\left\{\sqrt{\frac{\log p}{m^{*} n}}+\frac{\log (p)}{n}\right\},
$$

then $\widehat{B}_{n p}=B^{*}$ and $\widehat{G}_{n p}=G^{*}$, with probability higher than $1-c_{1} / p$. 
Obviously, Proposition C.3 implies the second result of Theorem 3.2.

Proof of Proposition C.3. The general organization and the notation are the same as for Theorem A.4. Indeed, analogously to A.16, we shall prove that

(C.8) $\left\langle W_{1}+W_{2}+W_{3}+n(\Gamma-\widehat{\Gamma}), B^{*}-B\right\rangle>0$, for all $B \in \mathcal{O}_{b}$ such that $\operatorname{supp}(B) \nsubseteq \operatorname{supp}\left(B^{*}\right)$,

with high probability. In fact, we still use the same bounds for $\left\langle W_{1}, B^{*}-B\right\rangle,\left\langle W_{2}, B^{*}-B\right\rangle$, and $\left\langle n(\Gamma-\widehat{\Gamma}), B^{*}-B\right\rangle$ as in the proof of Theorem A.5 and simply specify them for $R=0$ (exact $G$-block) model and $|\widehat{\Gamma}-\Gamma|_{V} \leq c|\Gamma|_{\infty} \sqrt{\log (p) / n}$ (Proposition A.10). This is possible since, Lemmas A.7 and A.9 do not require any bound on $\Delta\left(C^{*}\right)$. As for Lemma A.6, one easily checks by looking at the proof that its conclusion is valid for $R=0$ under the conditions $\log (p) \leq c n$ and $\Delta\left(C^{*}\right) \geq|\Gamma|_{\infty} \sqrt{\log (p) /\left(n m^{*}\right)}$.

It turns out that the suboptimal rate $\sqrt{\frac{p}{n m^{* 2}}}+\frac{p}{n m^{*}}$ in Theorem 5.3 is only due to the control $\left\langle W_{3}, B^{*}-B\right\rangle$ over $B \in \mathcal{C}$ in Lemma A.8. We provide here an alternative control for $B \in \mathcal{O}_{b}$.

Lemma C.4. With probability higher than $1-1 /$ p, it holds that

$$
\left|\left\langle W_{3}, B^{*}-B\right\rangle\right| \leq c\|\Gamma\|_{o p}\left(\sqrt{\frac{n \log (p)}{m}}+\log (p)\right) \sum_{j \neq k}\left|B_{G_{j} G_{k}}\right|_{1},
$$

uniformly over all $B \in \mathcal{O}_{b}$.

As in the proof of Theorem A.4, we gather the bounds of $\left\langle W_{i}, B^{*}-B\right\rangle$ for $i=1,2,3$ and $\left\langle\Gamma-\widehat{\Gamma}, B^{*}-B\right\rangle$ to derive a lower bound of $\left\langle W, B^{*}-B\right\rangle$, which turns out to be positive according to (C.7).

Proof of Lemma C.4. For a fixed $B \in \mathcal{O}_{b}$, the random variable $U_{B}=\left\langle W_{3}, B^{*}-B\right\rangle$ is distributed as a zero mean quadratic form of Gaussian random variables:

$$
\begin{aligned}
\left\langle W_{3}, B^{*}-B\right\rangle & =\sum_{i=1}^{n} X_{i}^{t}\left(I-B^{*}\right)\left(B^{*}-B\right)\left(I-B^{*}\right) X_{i}-\left\langle\Gamma,\left(I-B^{*}\right)\left(B^{*}-B\right)\left(I-B^{*}\right)\right\rangle \\
& =-\sum_{i=1}^{n} X_{i}^{t}\left(I-B^{*}\right) B\left(I-B^{*}\right) X_{i}+\left\langle\Gamma,\left(I-B^{*}\right) B\left(I-B^{*}\right)\right\rangle
\end{aligned}
$$

We control the deviations of $U_{B}$ using Lemma 1 in [5]. Since the covariance of $\left(I-B^{*}\right) X_{i}$ is $\left(I-B^{*}\right) \Gamma\left(I-B^{*}\right)$, it follows that, with probability higher than $1-e^{-t}$,

$$
\begin{aligned}
\left|\left\langle W_{3}, B^{*}-B\right\rangle\right| & \leq\left\|\Gamma^{1 / 2}\left(I-B^{*}\right)\left(B-B^{*}\right)\left(I-B^{*}\right) \Gamma^{1 / 2}\right\|_{F} 2 \sqrt{n t}+2\left\|\Gamma^{1 / 2}\left(I-B^{*}\right) B\left(I-B^{*}\right) \Gamma^{1 / 2}\right\|_{o p} t \\
& \leq\|\Gamma\|_{o p}\left(2\left\|B-B^{*}\right\|_{F} \sqrt{n t}+2 t\right) \\
& \leq\|\Gamma\|_{o p}\left(2 \sqrt{\frac{\left|B-B^{*}\right|_{1} n t}{m^{*}}}+2 t\right) \\
(\text { C.10) } & \leq\|\Gamma\|_{o p}\left(2 \sqrt{2\left(\sum_{j \neq k}\left|B_{G_{j}^{*} G_{k}^{*}}\right|_{1}\right) \frac{n t}{m^{*}}}+2 t\right)
\end{aligned}
$$


where we used in the second line that $B$ is an orthogonal projector, in the third line that the entries of $B$ and $B^{*}$ are either equal to one or $1 / \mathrm{m}^{*}$ and in the last lines that the sum of each row of $B$ and $B^{*}$ is equal to one.

Let us now take an union bound over all $B \in \mathcal{O}_{b}$. Given $B \in O_{b}$ associated to a balanced partition $G=\left(G_{1}, \ldots, G_{k}\right)$, define $\delta_{G^{*}, G}$ the $K \times K$ matrix of general entry $\left[\delta_{G^{*}, G}\right]_{i, j}=\left|G_{i}^{*} \cap G_{j}\right|$. Note that $\delta_{G^{*}, G}$ depends on the ordering of the groups in $G$. This is why we introduce $\delta_{B^{*}, B}$ as $\delta_{G^{*}, G^{\prime}}$ for $G^{\prime}$ minimizing $\left|\delta_{G^{*}, G^{\prime}}-\operatorname{Diag}\left(\delta_{G^{*}, G^{\prime}}\right)\right|_{\infty}$ over all reordering of the groups in $G$. Since the size of the groups of $B^{*}$ and $B$ are all equal to $m^{*}$, we claim that the largest non-diagonal entry of $\delta_{B^{*}, B}$ is smaller than $2 m^{*} / 3$.

Since $\left|\delta_{B^{*}, B}-\operatorname{Diag}\left(\delta_{B^{*}, B}\right)\right|_{1}=\sum_{k}\left|G_{k}^{*} \backslash G_{k}\right|$, the number of matrices $B$ in $\mathcal{O}_{b}$ such that $\mid \delta_{B^{*}, B}-$ $\left.\operatorname{Diag}\left(\delta_{B^{*}, B}\right)\right|_{1}=m$ is upper-bounded by the number of choices of $m$ elements among $p$ times the number of choices of a new group for each of these $m$ elements. There are less than $p^{m}(K-1)^{m}$ such choices.

Taking a union bound, it then follows from (C.10) that with probability higher than $1-1 / p$, we have

$$
\begin{aligned}
& \frac{\left|\left\langle W_{3}, B^{*}-B\right\rangle\right|}{\|\Gamma\|_{o p}} \\
& \leq c\left[\sqrt{\left(\sum_{j \neq k} \mid B_{G_{j}^{*} G_{k}^{*} \mid}\right)\left|\delta_{B^{*}, B}-\operatorname{Diag}\left(\delta_{B^{*}, B}\right)\right|_{1} \frac{n \log (p)}{m^{*}}}+\left|\delta_{B^{*}, B}-\operatorname{Diag}\left(\delta_{B^{*}, B}\right)\right|_{1} \log (p)\right] .
\end{aligned}
$$

To conclude, it remains to bound $\left|\delta_{B^{*}, B}-\operatorname{Diag}\left(\delta_{B^{*}, B}\right)\right|_{1}$ in terms of $\sum_{j \neq k}\left|B_{G_{j}^{*} G_{k}^{*}}\right|_{1}$. To alleviate the notation, we write $\delta$ for $\delta_{B^{*}, B}$. Given $j, k$, we have

$$
\left|B_{G_{j}^{*} G_{k}^{*}}\right|_{1}=\sum_{l=1}^{K} \frac{1}{m^{*}} \delta_{j l} \delta_{k l}
$$

so that summing over all $j \neq k$,

$$
\sum_{j \neq k}\left|B_{G_{j}^{*} G_{k}^{*}}\right|_{1}=\sum_{j, l} \delta_{j l}\left(1-\frac{\delta_{j l}}{m^{*}}\right)=\sum_{j \neq l} \delta_{j l}\left(\frac{\delta_{l l}}{m^{*}}+1-\frac{\delta_{j l}}{m^{*}}\right) .
$$

Since the off-diagonal entries of $\delta_{B^{*}, B}$ are smaller than $2 m^{*} / 3$,

$$
\sum_{j \neq k}\left|B_{G_{j}^{*} G_{k}^{*}}\right|_{1} \geq \frac{1}{3} \sum_{j \neq l} \delta_{j l}=\frac{1}{3}\left|\delta_{B^{*}, B}-\operatorname{Diag}\left(\delta_{B^{*}, B}\right)\right|_{1} .
$$

Together with (C.11), this concludes the proof of the lemma.

\section{APPENDIX D: RESULTS FOR THE COD ESTIMATOR: SECTIONS 4 AND 6.2}

We give below the proofs of Theorems 4.1, 6.2 and Proposition 4.2.

D.1. Proof of Theorems 4.1 and 6.2. In order to avoid cluttered notation, in all this subsection, we write $G$ for $G^{*}$ (resp. $\left.G[K]\right)$ and $m$ for $m^{*}$ (resp. $m[K]$ ).

The proof consists in the application of Lemma D.1 and Lemma D.2 proved below. 
Lemma D.1. Consider any partition $G$. Let us set $\tau=\max _{a, b, c=1, \ldots, p} \mid \widehat{\operatorname{cor}}\left(X_{a}-X_{b}, X_{c}\right)-$ $\operatorname{cor}\left(X_{a}-X_{b}, X_{c}\right) \mid$,

$$
\mu=\max _{\substack{G \\ a \sim b}} \operatorname{SCOD}(a, b) \text {, and } \eta=\min _{\substack{G \\ a \propto b}} \operatorname{sCOD}(a, b) \text {, where } \operatorname{sCOD}(a, b):=\max _{c \neq a, b}\left|\operatorname{cor}\left(X_{a}-X_{b}, X_{c}\right)\right| \text {. }
$$

Then, under the condition $\mu+\tau \leq \alpha<\eta-\tau$, the COD algorithm exactly recovers the partition $G$.

The control on the size of $\tau$ is given below.

Lemma D.2. Under Assumption 1, there exist two constants $c_{1}, c_{2}$ such that

$$
\tau:=\max _{a, b, c=1, \ldots, p}\left|\widehat{\operatorname{cor}}\left(X_{a}-X_{b}, X_{c}\right)-\operatorname{cor}\left(X_{a}-X_{b}, X_{c}\right)\right| \leq c_{1} L^{2} \sqrt{\frac{\log (p)}{n}},
$$

with probability at least $1-c_{2} / p$.

To conclude Theorem 4.1, we apply the two lemmas with $G=G^{*}$, implying that $\mu=0$ and $\eta \geq \operatorname{MCOD}(\Sigma) /\left(2|\Sigma|_{\infty}\right)$. To conclude Theorem 6.2, we remark that $\eta \geq \operatorname{MCOD}(\Sigma, G) /\left(2|\Sigma|_{\infty}\right)$, and $\mu \leq \sqrt{2}|R|_{\infty} / \lambda_{\min }(\Sigma)$.

Proof of Lemma D.1. First, we notice that,

$$
\widehat{\operatorname{sCOD}}(a, b)-\tau \leq \operatorname{sCOD}(a, b) \leq \widehat{\operatorname{sCOD}}(a, b)+\tau .
$$

We then observe that

$$
a \stackrel{G}{\sim} b \quad \Longrightarrow \quad \operatorname{sCOD}(a, b) \leq \mu \quad \Longrightarrow \quad \widehat{\operatorname{sCOD}}(a, b) \leq \mu+\tau
$$

and

$$
a \stackrel{G}{\nsim} b \Longrightarrow \operatorname{sCOD}(a, b) \geq \eta \quad \widehat{\operatorname{sCOD}}(a, b) \geq \eta-\tau .
$$

In particular, under the condition $\mu+\tau \leq \alpha<\eta-\tau$, we have

$$
a \stackrel{G}{\sim} b \Longleftrightarrow \widehat{\operatorname{SCOD}}(a, b) \leq \alpha
$$

Let us prove Lemma D.1 by induction on $l$. We consider the algorithm at some step $l$ and assume that the algorithm was consistent up to this step, i.e. $\widehat{G}_{j}=G_{k\left(a_{j}\right)}$ for $j=1, \ldots, l-1$.

If $|S|=1$, then it directly follows that $\widehat{G}=G$. Assume now that $|S|>1$.

(i) If $\widehat{\operatorname{sCOD}}\left(a_{l}, b_{l}\right)>\alpha$, then according to (D.2) no $b \in S$ is in the same group as $a_{l}$. Since the algorithm has been consistent up to step $l$, it means that $a_{l}$ is a singleton and $\widehat{G}_{l}:=\left\{a_{l}\right\}=G_{k\left(a_{l}\right)}$.

(ii) If $\widehat{\operatorname{sCOD}}\left(a_{l}, b_{l}\right) \leq \alpha$, then $a_{l} \stackrel{G}{\sim} b_{l}$ according to (D.2). The equivalence (D.2) furthermore ensures that $\widehat{G}_{l}=S \cap G_{k\left(a_{l}\right)}$. Since the algorithm has been consistent up to this step we have $G_{k\left(a_{l}\right)} \subset S$ and hence $\widehat{G}_{l}=G_{k\left(a_{l}\right)}$.

To conclude, the algorithm remains consistent at step $l$ and the Lemma D.1 follows by induction.

Proof of Lemma D.2. We start with the following lemma.

Lemma D.3. Let $W$ be a random vector in $\mathbf{R}^{d}$, then

$$
\max _{a, b=1, \ldots, d}\left|\widehat{\operatorname{cor}}\left(W_{a}, W_{b}\right)-\operatorname{cor}\left(W_{a}, W_{b}\right)\right| \leq 2 \max _{a, b=1, \ldots, d} \frac{\left|\widehat{\operatorname{cov}}\left(W_{a}, W_{b}\right)-\operatorname{cov}\left(W_{a}, W_{b}\right)\right|}{\sqrt{\operatorname{var}\left(W_{a}\right) \operatorname{var}\left(W_{b}\right)}} .
$$


Proof of Lemma D.3. Since both sides of (D.3) are invariant by rescaling any of the $W_{a}$, we can always assume that $\operatorname{var}\left(W_{a}\right)=1$ for all $a=1 \ldots, d$. We denote by $R$ and $\widehat{R}$ the correlation matrix of $W$ and its empirical counterpart, and by $S$ and $\widehat{S}$ its covariance matrix and its empirical counterpart. Since $\operatorname{var}\left(W_{a}\right)=1$ for all $a=1 \ldots, d$, we have $R=S$, and the triangular inequality gives

$$
\begin{aligned}
\left|\widehat{R}_{a b}-R_{a b}\right| & =\left|\widehat{R}_{a b}\left(1-\left(\widehat{S}_{a a} \widehat{S}_{b b}\right)^{1 / 2}\right)+\widehat{S}_{a b}-S_{a b}\right| \\
& \leq\left|\widehat{R}_{a b}\right|\left|1-\left(\widehat{S}_{a a} \widehat{S}_{b b}\right)^{1 / 2}\right|+\left|\widehat{S}_{a b}-S_{a b}\right|
\end{aligned}
$$

We notice that

$$
\left|1-\left(\widehat{S}_{a a} \widehat{S}_{b b}\right)^{1 / 2}\right| \leq\left|1-\widehat{S}_{a a}\right| \vee\left|1-\widehat{S}_{b b}\right|=\left|S_{a a}-\widehat{S}_{a a}\right| \vee\left|S_{b b}-\widehat{S}_{b b}\right| .
$$

Since $\left|\widehat{R}_{a b}\right| \leq 1$, we conclude that for any $a, b \in\{1, \ldots, d\}$

$$
\begin{aligned}
\left|\widehat{R}_{a b}-R_{a b}\right| & \leq\left|\widehat{R}_{a b}\right|\left(\left|S_{a a}-\widehat{S}_{a a}\right| \vee\left|S_{b b}-\widehat{S}_{b b}\right|\right)+\left|\widehat{S}_{a b}-S_{a b}\right| \\
& \leq 2|\widehat{S}-S|_{\infty}
\end{aligned}
$$

The proof of Lemma D.3 is complete.

We now apply previous lemma to the random vector $W$ in dimension $d=p(p+1) / 2$ gathering all the $X_{c}$ for $c=1, \ldots, p$ and all the $X_{a}-X_{b}$ for $1 \leq a<b \leq p$. We complete the proof of Lemma D.2 by combining (D.3) with (F.3) and by noticing that we always have $\tau \leq 2$ so the term with the square-root is dominant.

D.2. Proof of Proposition 4.2. For any $a<b$, we introduce the notation $\epsilon_{a b}^{(i)}=\widehat{\Delta}_{a b}^{(i)}-\Delta_{a b}$ for $i=1,2$. Let $\left.E H(G)=: \mathbb{E}^{(2)}[\mathcal{H} G)\right]$. For any partition $G$, we have

$$
\begin{aligned}
E H(G)-E H\left(G^{*}\right)= & \sum_{\substack{G^{*} b \\
\sim}} \mathbf{1}_{\substack{G \\
\sim}} \mathbb{E}^{(2)}\left[\left|\epsilon_{a b}^{(1)}-\epsilon_{a b}^{(2)}\right|_{\infty}^{2}-\left|\epsilon_{a b}^{(1)}\right|_{\infty}^{2}\right] \\
& +\sum_{\substack{G^{*} b \\
a \sim b}} \mathbf{1}_{\substack{G \\
\sim}} \mathbb{E}^{(2)}\left[\left|\Delta_{a b}+\epsilon_{a b}^{(1)}\right|_{\infty}^{2}-\left|\epsilon_{a b}^{(1)}-\epsilon_{a b}^{(2)}\right|_{\infty}^{2}\right] .
\end{aligned}
$$

Let us prove that both terms are positive both in expectation and in probability with respect to $\mathbf{P}^{(2)}$.

The property (P1) ensures that since $\mathbb{E}\left[\epsilon_{a b}^{(2)}\right]=0$. So, for the first sum, Jensen inequality implies that

$$
\mathbb{E}^{(2)}\left[\left|\epsilon_{a b}^{(1)}-\epsilon_{a b}^{(2)}\right|_{\infty}^{2}\right] \geq\left|\epsilon_{a b}^{(1)}-\mathbb{E}^{(2)}\left[\epsilon_{a b}^{(2)}\right]\right|_{\infty}^{2}=\left|\epsilon_{a b}^{(1)}\right|_{\infty}^{2}
$$

Hence the first sum is always non-negative.

Let us now consider the second sum where $a \stackrel{G^{*}}{\nsim} b$ but $a \stackrel{G}{\sim} b$. The inequality

$$
\left|\Delta_{a b}\right|_{\infty}^{2} \leq\left(\left|\Delta_{a b}+\epsilon_{a b}^{(1)}\right|_{\infty}+\left|\epsilon_{a b}^{(1)}\right|_{\infty}\right)^{2} \leq 2\left|\Delta_{a b}+\epsilon_{a b}^{(1)}\right|_{\infty}^{2}+2\left|\epsilon_{a b}^{(1)}\right|_{\infty}^{2},
$$

ensures the lower bound

$$
\mathbb{E}^{(2)}\left[\left|\Delta_{a b}+\epsilon_{a b}^{(1)}\right|_{\infty}^{2}-\left|\epsilon_{a b}^{(1)}-\epsilon_{a b}^{(2)}\right|_{\infty}^{2}\right] \geq \frac{1}{2}\left|\Delta_{a b}\right|_{\infty}^{2}-2\left|\epsilon_{a b}^{(1)}\right|_{\infty}^{2}-\mathbb{E}^{(2)}\left[\left|\epsilon_{a b}^{(2)}\right|_{\infty}^{2}\right]
$$


The Lemma D.3 together with the Corollary F.2 of Hanson-Wright inequality ensures that

$$
\max _{a, b, c=1, \ldots, p}\left|\left[\epsilon_{a b}^{(i)}\right]_{c}\right|^{2} \leq \min \left(2, c L^{2}\left(\sqrt{\frac{\log (p)}{n}}+\frac{\log (p)}{n}\right)\right)^{2} \leq 4 c^{2} L^{4}|\Sigma|_{\infty}^{2} \frac{\log (p)}{n}
$$

both with probability larger than $1-4 / p$ and in expectation with respect to $\mathbf{P}^{(i)}$. Since

$$
4\left|\Delta_{a b}\right|_{\infty}^{2} \geq \operatorname{MCOD}(\Sigma)^{2} /|\Sigma|_{\infty}^{2}>c_{1}^{2} L^{4} \log (p) / n
$$

the proof of Proposition 4.2 is complete.

\section{APPENDIX E: ANALYSIS OF CORRECTED SPECTRAL CLUSTERING: SECTION 5.4}

In this section, we prove Proposition 5.6. We recall that we work here with the decomposition $\Sigma=A C^{*} A^{t}+\Gamma$ where $\Gamma$ is diagonal. As in the previous section, we write $m$ for $m^{*}$ and $G$ for $G^{*}$.

An alternative formulation of an approximate $K$-means is the following. Let $\eta>1$ be a given positive number. Denote $\mathcal{A}_{p, K}$ the collection of membership matrices, that is $p \times K$ binary matrices whose rows contain exactly one non-zero entry. Note that a membership matrix $A \in \mathcal{A}_{p, K}$ defines a partition $G$. Given a $p \times K$ matrix $\widehat{U}$, the membership matrix $\widehat{A}$ is said to be an $\eta$-approximation $K$-means problem on $\widehat{U}$ if there exists a $K \times K$ matrix $\widehat{Q}$ such that

$$
\|\widehat{U}-\widehat{A} \widehat{Q}\|_{F}^{2} \leq \eta \min _{A \in \mathcal{A} p, k} \min _{Q}\|\widehat{U}-A Q\|_{F}^{2}
$$

The partition $\widehat{G}$ is then the partition corresponding to $\widehat{A}$.

The proof is based on the following Lemma by Lei and Rinaldo [6].

Lemma E.1. Let $M$ be any matrix of the form $M=A Q$ where $A \in \mathcal{A}_{p, K}$ is a membership matrix associated to $G$ and $Q \in \mathbf{R}^{K \times q}$, and denote by $\delta$ the minimal distance between two rows of $Q$. Then, there exists a constant $c_{\eta}$, such that, for any matrix $M^{\prime}$ fulfilling $\left\|M-M^{\prime}\right\|_{F}^{2}<m \delta^{2} / c_{\eta}$, the classification of the rows of $M^{\prime}$ by an $\eta$-approximate $K$-means provides a clustering $\widehat{G}$ fulfilling

$$
\bar{L}(\hat{G}, G) \leq c_{\eta} \frac{\left\|M-M^{\prime}\right\|_{F}^{2}}{m \delta^{2}} .
$$

We also need the following bounds

Lemma E.2. We have $\|\Sigma\|_{o p} \geq m\left\|C^{*}\right\|_{o p}$ and $|\Gamma|_{\infty} \leq\|\Sigma\|_{o p}$.

In view of this Lemma, we obtain $\|\Sigma\|_{o p} \geq m\left\|C^{*}\right\|_{o p} \geq m \lambda_{K}\left(C^{*}\right)$. Then, Condition (5.14) of Proposition 5.6 enforces that

$$
\frac{\operatorname{Re}(\Sigma) \vee \log (p)}{n} \leq 1 / c_{\eta}^{2}
$$

Let $U$ be a $K \times p$ matrix which gathers the eigenvectors of $A C^{*} A^{t}$ associated to the $K$ leading eigenvalues. The associated eigenvectors are block constant. Therefore $U_{0}=A Q_{0}$, and since $A^{t} A=$ $m I$, the matrix $\sqrt{m} Q_{0}$ is orthogonal.

We apply Lemma E.1 with $M^{\prime}=\widehat{U}$ and $M=U_{0} \widehat{O}$, where $\widehat{O}$ is a $K \times K$ orthogonal matrix to be chosen. We have $M=A Q$ with $\sqrt{m} Q=\sqrt{m} Q_{0} \widehat{O}$ orthogonal. In particular, the minimal distance between two rows of $Q$ is $\delta=\sqrt{2 / m}$. Lemma E.1 ensures that

$$
\bar{L}\left(\hat{G}_{S}, G\right) \leq c_{\eta} \frac{\left\|\widehat{U}-U_{0} \widehat{O}\right\|_{F}^{2}}{2}
$$


whenever the right-hand side is smaller than 1. By Davis-Kahan inequality (e.g. [6]), there exists an orthogonal matrix $\widehat{O}$ such that

$$
\left\|\widehat{U}-U_{0} \widehat{O}\right\|_{F}^{2} \leq \frac{8 K\left\|\widetilde{\Sigma}-A C^{*} A^{t}\right\|_{o p}^{2}}{m^{2} \lambda_{K}^{2}\left(C^{*}\right)} .
$$

We can upper-bound the operator norm of $\widetilde{\Sigma}-A C^{*} A^{t}$ by

$$
\left\|\widetilde{\Sigma}-A C^{*} A^{t}\right\|_{o p} \leq\|\widehat{\Sigma}-\Sigma\|_{o p}+\|\widehat{\Gamma}-\Gamma\|_{o p} .
$$

According to Theorem 9 in [4], there exists a constant $c>0$ such that, with probability at least $1-1 / p$

$$
\begin{aligned}
\|\widehat{\Sigma}-\Sigma\|_{o p} & \leq c_{L}\|\Sigma\|_{o p}\left(\sqrt{\frac{\operatorname{Re}(\Sigma)}{n}} \bigvee \frac{\operatorname{Re}(\Sigma)}{n} \bigvee \sqrt{\frac{\log (p)}{n}} \bigvee \frac{\log (p)}{n}\right) \\
& \leq c_{L}\|\Sigma\|_{o p}\left(\sqrt{\frac{\operatorname{Re}(\Sigma)}{n}} \bigvee \sqrt{\frac{\log (p)}{n}}\right),
\end{aligned}
$$

where we used (E.2) in the second line.

Then, using that $\|\widehat{\Gamma}-\Gamma\|_{o p}=|\widehat{\Gamma}-\Gamma|_{\infty}$ and Proposition A.10, together with $|\Gamma|_{\infty} \leq\|\Sigma\|_{o p}$ (Lemma E.2 above), we obtain the inequality

$$
\left\|\widetilde{\Sigma}-A C^{*} A^{t}\right\|_{o p} \leq c_{L}\|\Sigma\|_{o p}\left(\sqrt{\frac{R e(\Sigma)}{n}} \bigvee \sqrt{\frac{\log (p)}{n}}\right),
$$

with probability at least $1-c / p$. So combining (E.3), with (E.4) and (E.5) we obtain the existence of $c_{\eta}^{\prime}>0$ such that we have

$$
\bar{L}\left(\hat{G}_{S}, G\right) \leq \frac{c_{\eta, L}^{\prime} K\|\Sigma\|_{o p}^{2}}{m^{2} \lambda_{K}\left(C^{*}\right)^{2}}\left(\sqrt{\frac{\operatorname{Re}(\Sigma)}{n}} \bigvee \sqrt{\frac{\log (p)}{n}}\right)^{2},
$$

with probability at least $1-c / p$, whenever the right-hand side is smaller than 1 . The proof of Proposition 5.6 follows.

Proof OF Lemma 5.5. We recall that $\widehat{V}$ is the $p \times K$ matrix stacking the $K$ leading eigenvectors of $\widehat{\Sigma}$. We first prove that the matrix $\widehat{V} \widehat{V}^{t}$ is solution of the SDP in Lemma 5.5.

Let us write $\widehat{\Sigma}=\widetilde{V} \widetilde{D} \widetilde{V}^{t}$ for a diagonalisation of $\widehat{\Sigma}$ with $\widetilde{V}$ orthogonal and $\widetilde{D}_{11} \geq \ldots \geq \widetilde{D}_{p p} \geq 0$. We observe that $\langle\widehat{\Sigma}, B\rangle=\left\langle\widetilde{D}, \widetilde{V}^{t} B \widetilde{V}\right\rangle$, and that $B \in \overline{\mathcal{C}}$ iff $\widetilde{V}^{t} B \widetilde{V} \in \overline{\mathcal{C}}$ since the matrix $\widetilde{B}=\widetilde{V} \widetilde{V}^{t} B$ has the same eigenvalues as $B$. We observe also that $\widehat{V} \widehat{V}^{t}=\widetilde{V} \Pi_{K} \widetilde{V}^{t}$, where $\Pi_{K}$ is the diagonal matrix, with 1 on the first $K$ diagonal elements and 0 on the $p-K$ remaining ones. So proving that $\bar{B}=\widehat{V} \widehat{V}^{t}$ is solution of the SDP in Lemma 5.5 is equivalent to proving that

$$
\Pi_{K}=\underset{\widetilde{B} \in \overline{\mathcal{C}}}{\operatorname{argmax}}\langle\widetilde{D}, \widetilde{B}\rangle \text {. }
$$

Let us prove this result.

To start with, we notice that

$$
\sum_{k=1}^{K} \widetilde{D}_{k k}=\max _{0 \leq \widetilde{B}_{k k} \leq 1 ; \sum_{k} \widetilde{B}_{k k}=K}\langle\widetilde{D}, \widetilde{B}\rangle .
$$


Since the condition $I \succcurlyeq \widetilde{B} \succcurlyeq 0$ enforces $0 \leq \widetilde{B}_{k k} \leq 1$, we have $\overline{\mathcal{C}} \subset\left\{B: 0 \leq \widetilde{B}_{k k} \leq 1 ; \sum_{k} \widetilde{B}_{k k}=K\right\}$ and then

$$
\max _{\widetilde{B} \in \overline{\mathcal{C}}}\langle\widetilde{D}, \widetilde{B}\rangle \leq \sum_{k=1}^{K} \widetilde{D}_{k k}=\left\langle\widetilde{D}, \Pi_{K}\right\rangle .
$$

Hence $\Pi_{K}$ is solution to the above maximisation problem and $\bar{B}=\widetilde{V} \Pi_{K} \widetilde{V}^{t}=\widehat{V} \widehat{V}^{t}$.

To conclude the proof, we notice that $\widehat{V}_{a} \widehat{V}^{t}$ is an orthogonal transformation of $\widehat{V}_{a}$, so we obtain the same results when applying a rotationally invariant clustering algorithm to the rows of $\widehat{V}$ and to the rows of $\widehat{V} \widehat{V}^{t}$.

Proof of Lemma E.2. We claim that, for each group $G_{k}$, there is at most one negative value $\Gamma_{a a}$ with $a \in G_{k}$. When it exists, this negative value also satisfies $\left|\Gamma_{a a}\right| \leq \min _{b \in G_{k} \backslash\{a\}} \Gamma_{b b}$.

Indeed, take any $a, b \in G_{k}$ such that $\Gamma_{a a}<0$ (if it exists). Then, the corresponding $2 \times 2$ submatrix of $\Sigma$

$$
\left(\begin{array}{cc}
\Sigma_{a a} & \Sigma_{a b} \\
\Sigma_{b a} & \Sigma_{b b}
\end{array}\right)=\left(\begin{array}{cc}
C_{k k}-\left|\Gamma_{a a}\right| & C_{k k} \\
C_{k k} & C_{k k}+\Gamma_{b b}
\end{array}\right)
$$

is positive semi-definite, which enforces $\Gamma_{b b} \geq\left|\Gamma_{a a}\right|$.

Let us now prove the first result of the lemma. Since $A^{t} A=m I$, we have $\|\Sigma\|_{o p} \geq\left\|A^{t} \Sigma A\right\|_{o p} / m=$ $\left\|m C+A^{t} \Gamma A / m\right\|_{o p}$. Since $A^{t} \Gamma A / m$ is a diagonal matrix with entries $\sum_{a \in G_{k}} \Gamma_{a a} /\left|G_{k}\right|$, for $k=$ $1, \ldots, K$, we finally have

$$
\|\Sigma\|_{o p} \geq m\left\|C^{*}\right\|_{o p}+\min _{k} \sum_{a \in G_{k}} \frac{1}{\left|G_{k}\right|} \Gamma_{a a} \geq m\left\|C^{*}\right\|_{o p},
$$

where the second inequality is a consequence of the above claim. As for the second result, we observe that $|\Gamma|_{\infty}$ is achieved by some $\Gamma_{a a}>0$. Then, $\|\Sigma\|_{o p} \geq C_{k(a) k(a)}^{*}+\Gamma_{a a} \geq|\Gamma|_{\infty}$ since Condition (5.14) of Proposition 5.6 enforces that $C$ is positive.

\section{APPENDIX F: DEVIATION INEQUALITIES}

The following lemma provides deviation inequalities for quadratic form of Sub-Gaussian and Bernstein-type random variables that we used in the proofs presented above and in the main text [2]. For the first inequality we use [10], whereas the second one is proved in [1].

Lemma F.1 (Hanson-Wright Inequalities). There exists two positive constants $c$ and $c^{\prime}$ such that the following holds for all $n \times n$ matrices $A$. Let $\xi=\left(\xi_{1}, \xi_{2}, \ldots, \xi_{n}\right)^{t}$ denote a vector of independent zero-mean random variables with respectives variances $\sigma_{1}^{2}, \ldots, \sigma_{n}^{2}$.

(i) Assume that all variables $\xi_{i}$ follow sub-Gaussian distributions with $\max _{i=1, \ldots, n}\left\|\xi_{i}\right\|_{\psi_{2}} \leq L$ for some $L>0$. Then, for all $t>0$

$$
\mathbb{P}\left[\xi^{t} A \xi-\mathbb{E}\left[\xi^{t} A \xi\right]>c L^{2}\left(\|A\|_{2} \sqrt{t}+c\|A\|_{o p} t\right)\right] \leq e^{-t} .
$$

(ii) Assume that, for some $M>0$, $\xi$ satisfies $\max _{i=1, \ldots, n}\left|\xi_{i}\right| \leq M$ almost surely. Then, for all $t>0$

$$
\mathbb{P}\left[\xi^{t} A \xi-\mathbb{E}\left[\xi^{t} A \xi\right]>c^{\prime}\left(M\left\|A D_{\sigma}\right\|_{2} \sqrt{t}+c M^{2}\|A\|_{o p} t\right)\right] \leq e^{-t}
$$

where $D_{\sigma}=\operatorname{Diag}\left(\sigma_{1}, \ldots, \sigma_{n}\right)$. 
The following corollary will be useful for controlling cross-products of random variables.

Corollary F.2. Let $\mathbf{X}$ denotes the observation matrix and let $u, v$ be two p-dimensional vectors. Under Assumption 1, for any $t>0$, with probability at least $1-4 e^{-t}$, we have

$$
\left|\frac{1}{n}\langle\mathbf{X} u, \mathbf{X} v\rangle-u^{t} \Sigma v\right| \leq c L^{2} \sqrt{u^{t} \Sigma u v^{t} \Sigma v}\left(\sqrt{\frac{t}{n}}+\frac{t}{n}\right) .
$$

Proof of Corollary F.2. Let us set $\alpha=\left(v^{t} \Sigma v / u^{t} \Sigma u\right)^{1 / 4}$. We first observe that

$$
\langle\mathbf{X} u, \mathbf{X} v\rangle=\frac{1}{4}\left(\left\|\alpha \mathbf{X} u+\alpha^{-1} \mathbf{X} v\right\|^{2}-\left\|\alpha \mathbf{X} u-\alpha^{-1} \mathbf{X} v\right\|^{2}\right) .
$$

Since for any $w \in \mathbf{R}^{p}$, we have $\left\|X^{t} w\right\|_{\psi_{2}} \leq L\left(w^{t} \Sigma w\right)^{1 / 2}$, Hanson-Wright inequality gives that with probability at least $1-4 e^{-t}$, we have both

$$
\begin{aligned}
& \left|\left\|\alpha \mathbf{X} u+\alpha^{-1} \mathbf{X} v\right\|^{2}-\mathbb{E}\left[\left\|\alpha \mathbf{X} u+\alpha^{-1} \mathbf{X} v\right\|^{2}\right]\right| \leq c L^{2}\left(\alpha u+\alpha^{-1} v\right)^{t} \Sigma\left(\alpha u+\alpha^{-1} v\right)(\sqrt{n t}+t) \\
& \left|\left\|\alpha \mathbf{X} u-\alpha^{-1} \mathbf{X} v\right\|^{2}-\mathbb{E}\left[\left\|\alpha \mathbf{X} u-\alpha^{-1} \mathbf{X} v\right\|^{2}\right]\right| \leq c L^{2}\left(\alpha u-\alpha^{-1} v\right)^{t} \Sigma\left(\alpha u-\alpha^{-1} v\right)(\sqrt{n t}+t) .
\end{aligned}
$$

Combining the two bounds, we get

$$
\left|\frac{1}{n}\langle\mathbf{X} u, \mathbf{X} v\rangle-u^{t} \Sigma v\right| \leq \frac{1}{2} c L^{2}\left(\alpha^{2} u^{t} \Sigma u+\alpha^{-2} v^{t} \Sigma v\right)(\sqrt{t / n}+t / n) .
$$

Replacing $\alpha^{2}$ by its value $\alpha^{2}=\left(v^{t} \Sigma v / u^{t} \Sigma u\right)^{1 / 2}$, we get the desired result.

We have a similar result under Assumption 1-bis.

Lemma F.3. Let $\mathbf{X}$ denotes the observation matrix and let $u, v$ be two p-dimensional vectors. Under Assumption 1-bis, for any $t>0$, with probability at least $1-2 e^{-t}$, we have

$$
\left|\frac{1}{n}\langle\mathbf{X} u, \mathbf{X} v\rangle-u^{t} \Sigma v\right| \leq 2 M\left(\left(|v|_{1} \sqrt{u^{t} \Sigma u}\right) \wedge\left(|u|_{1} \sqrt{v^{t} \Sigma v}\right)\right) \sqrt{\frac{t}{n}}+3 M^{2}|u|_{1}|v|_{1} \frac{t}{n} .
$$

Proof of Lemma F.3. The variable $Z_{i}=\left(X_{i}^{t} u\right)\left(X_{i}^{t} v\right)-u^{t} \Sigma v$ fulfills $\left|Z_{i}\right| \leq B=2 M^{2}|v|_{1}|u|_{1}$ and

$$
\mathbb{E}\left[Z_{i}^{2}\right] \leq \mathbb{E}\left[\left(X_{i}^{t} u X_{i}^{t} v\right)^{2}\right] \leq M^{2} \min \left(|u|_{1}^{2} v^{t} \Sigma v,|v|_{1}^{2} u^{t} \Sigma u\right)
$$

The inequality (F.4) then simply follows from Bernstein inequality.

The two following lemmas control the behavior of empirical covariance matrices both under subGaussian and Bernstein-type distributional assumptions. The first one follows from [4, 12], whereas the second one can be found in the expository paper [11].

Lemma F.4. Consider a zero mean random vector $Y$ of size $p$ and invertible covariance matrix $\Sigma$. Assume that, for some $L>0,\left\|\Sigma^{-1 / 2} Y\right\|_{\psi_{2}} \leq L$. Then, for some constant $c_{L}>0$, the following holds. Given a n-sample $\left(Y_{1}, \ldots, Y_{n}\right)$, the empirical covariance matrix $\widehat{\Sigma}=\sum_{i=1}^{n} Y_{i} Y_{i}^{t} / n$ satisfies the following deviation inequality

$$
\mathbb{P}\left[\|\widehat{\Sigma}-\Sigma\|_{o p}>c_{L}\|\Sigma\|_{o p}\left[\sqrt{\frac{p}{n}}+\frac{p}{n}+\sqrt{\frac{t}{n}}+\frac{t}{n}\right]\right] \leq e^{-t},
$$

for any $t>0$. 
Lemma F.5 (Matrix Bernstein Inequality). Consider a zero mean random vector $Y$ of size $p$ and covariance matrix $\Sigma$. Assume that, for some $M>0, \max _{i=1, \ldots, p}\left|Y_{i}\right| \leq M$ almost surely. Given a n-sample $\left(Y_{1}, \ldots, Y_{n}\right)$, the empirical covariance matrix $\widehat{\Sigma}=\sum_{i=1}^{n} Y_{i} Y_{i}^{t} / n$ satisfies the following deviation inequality

$$
\mathbb{P}\left[\|\widehat{\Sigma}-\Sigma\|_{o p}>M \sqrt{2 \frac{p\|\Sigma\|_{o p}(t+\log (2 p))}{n}}+\frac{4 p M^{2}(t+\log (2 p))}{3 n}\right] \leq e^{-t},
$$

for any $t>0$.

\section{APPENDIX G: SIMULATION RESULTS}

In this section we verify numerically our theoretical findings and also illustrate the finite sample performance of our methods. The implementation of PECOK can be found at http://github. com/martinroyer/pecok/ and that of COD at http://CRAN.R-project.org/package=cord.

G.1. Simulation design. Recall our $G$-latent covariance $\Sigma=A C A^{t}+\Gamma$. Under various scenarios of $A$ and $\Gamma$ to be described momentarily, we consider the following models for $C$ :

- Model 1: $C=B^{T} B$ where $B$ is a random $(K-1) \times K$ matrix with independent entries. Each entry takes the value +1 and -1 with equal probability $0.5 \times K^{-1 / 2}$, and the value 0 with probability $1-K^{1 / 2}$.

- Model 2: $C=C^{t}-0.001 I$ where $C^{t}$ is generated by Model 1 .

The matrix $C$ is positive semi-definite in Model 1 and negative definite in Model 2. In the first two simulation scenarios (referred to as M1 and M2 thereafter), we set $C$ derived from Models 1-2 respectively, and specify $A$ to correspond to $K=10$ equal-size groups of variables (or equivalently $m=p / K)$.

In a third scenario (M1S), we specify $A$ such that it corresponds to the existence of 5 singletons, which are variables that form their own groups of size 1, respectively, and the remaining $K-5$ groups have equal-size, while $C$ is the same as M1.

In these first three scenarios, we employ diagonal $\Gamma=D$ where the $p$ diagonal entires of $D$ are random permutations of $\{0.5,0.5+1.5 /(p-1), \ldots, 2\}$.

In the fourth scenario (M1P), we consider the approximate $G$-block model by setting $\Gamma=D+R$ where $R=0.1 \cdot U^{t} U / \max \left(U^{t} U\right)$ and $U$ is a $p \times p$ matrix with iid random entries from a uniform distribution on $[-1,1]$. We run these four scenarios for two representative $p=200$ and $p=1600$, and for sample sizes $n=100,300, \ldots, 900$ unless noted otherwise. All simulations are repeated 100 times.

The goal of our methods is to create sub-groups of vectors of dimension $n$, from a given collection of $p$ vectors of observations, each of dimension $n$. This task can be viewed as that of clustering $p$ objects in $\mathbb{R}^{n}$. The existing data clustering algorithms are not tailored to recovering groups with this structure, but they can serve as comparative methods. We thus compare the performance of COD and PECOK with three popular clustering algorithms: K-means (Lloyd algorithm with random initialisations), Hierarchical Clustering (HC) and spectral clustering. We apply K-means on the columns of the $n \times p$ matrix of $n$ observations, and use the negative correlation as distance matrix in HC. The spectral clustering algorithm is discussed in Section 5.4, which does not correct for $\Gamma$. We use the standard K-means and $\mathrm{HC}$ algorithms in $\mathrm{R}$, and we also implement our COD algorithm in $\mathrm{R}$. We also include a variant of the COD algorithm suggested by a reviewer. This variant applies the connected component algorithm (as implemented in CRAN package igraph available at https://CRAN.R-project.org/package=igraph) to a graph converted from our proposed COD 
metric matrix thresholded at level $\alpha$, which will be referred to as COD-CC. The spectral clustering algorithm is based on the widely used Python package scikit-learn, and we implement our PECOK algorithm also using Python.

The three competing methods require specification of the number of groups $K$, and we will use the true $K=10$ to evaluate their oracle performance. For our proposed methods, we use the data-splitting approach proposed in Section 4.3 to determine $K$ in a data-adaptive fashion. We select either $K$ from a grid in PECOK or the $K$-related threshold $\alpha$ also from a grid in COD, using the two independent datasets of size $n$ proposed in Section 4.3. The grid for PECOK is $2,4, \ldots, 40$. Since the theoretical choice of $\alpha$ is proportional to $n^{-1 / 2} \log ^{1 / 2} p$, we use a grid of $\alpha /\left(n^{-1 / 2} \log ^{1 / 2} p\right)=0.25,0.5, \ldots, 5$ in COD.

G.2. Exact recovery performance and comparison. Figure 1 shows the average percentages of exact recovery across 100 runs by K-means, HC, COD, COD-CC, and PECOK when $n$ varies. Under one setting with a very large $p=1600$, PECOK did not complete computation within two weeks, and thus its numerical performance for large $p=1600$ was not reported in the figure. COD and COD-CC clearly outperform all other competing methods (K-means, HC and spectral clustering) when $n$ is about 300 or larger in all the scenarios. K-means and HC, even with the oracle choice of $K$ and large $n=900$, fail to recover the true groups exactly. COD-CC is better than COD for small $n=100$, there are almost no finite sample differences between COD and COD-CC for $n=900$, which is consistent with our theory showing that they share the same rates described before. COD-CC and COD have similar performance across almost all models, and COD-CC achieves close to 100\% recovery for smaller samples than COD under the model with singletons (M1S). Except for the model containing singletons (M1S), PECOK has the best performance for small $n=100$ and $p=200$, and achieves close to 100\% as COD and COD-CC for larger $n$. Under the singleton scenario M1S, COD-CC has the best performance for all $n$, while the difference between COD-CC and COD vanishes when $n=900$. Under this model, the recovery percentages of PECOK increase with $n$ but only reaches about $40 \%$ when $n=900$ and $p=200$. This is consistent with our theoretical results that PECOK is not expected to work well in the presence of singletons, while COD adapts to this situation. We also note that the competing methods used for comparison are able to recover clusters very close to the truth (measured by the adjusted rand index, or ARI), see Section G.5 for this partial recovery comparison.

G.3. The importance of correcting for $\boldsymbol{\Gamma}$ in PECOK. The step 1 of our PECOK algorithm is to estimate and correct for $\Gamma$. We illustrate the importance of this step by comparing its performance with two closely related methods, $\Gamma$-uncorrected PECOK and K-means, neither of which corrects for $\Gamma$. The $\Gamma$-uncorrected PECOK algorithm simply replaces the estimated $\hat{\Gamma}$ in the step 1 of PECOK by a zero matrix. We use the true $K$ as input to these two methods to assess their oracle performance under the true $K$, while the parameter $K$ in PECOK is selected by data-splitting as described before. To fix ideas, we use scenario M1 described before and set $p=200$. Figure 2(a) shows that the exact recovery percentages of K-means are close to zero, and those of $\Gamma$-uncorrected PECOK are smaller than $30 \%$, across all $n$. After correcting for $\Gamma$, PECOK yields close to $100 \%$ exact recovery when $n$ increases to 300 .

G.4. Comparison under varying $\boldsymbol{m}$. In this section we illustrate the finite sample performance of PECOK when $m$ varies. We use the simulation scenario M1 under $p=200$, and consider $m=50$ and $m=20$, for $n=60,80,100,150$, to compare the increasing trend in performance before reaching $100 \%$ when $n$ reaches 200 as shown in Figure 1 . The rest of the simulation set-up is the same as that of the previous section. As predicted by our theory, Figure 2(b) shows the percentages of exact recovery is better for a larger $m=50$, compared with $m=20$, for the same $\mathrm{n}$. 
Figure 1: Percentages of exact recovery by K-means (K-Oracle, medium green lines, triangle points), HC (H-Oracle, light blue lines, cross points), spectral clustering (S-Oracle, light yellow lines, upside-down triangle points), COD (dark red lines, circle points), COD-CC (light orange lines, plus points), PECOK (light pink lines, diamond points) across 100 runs of 4 scenarios described in the main text, when $p=200$ (solid lines) and $p=1600$ (dashed lines). All standard errors are smaller than $5 \%$.

(a) M1



(c) M1S

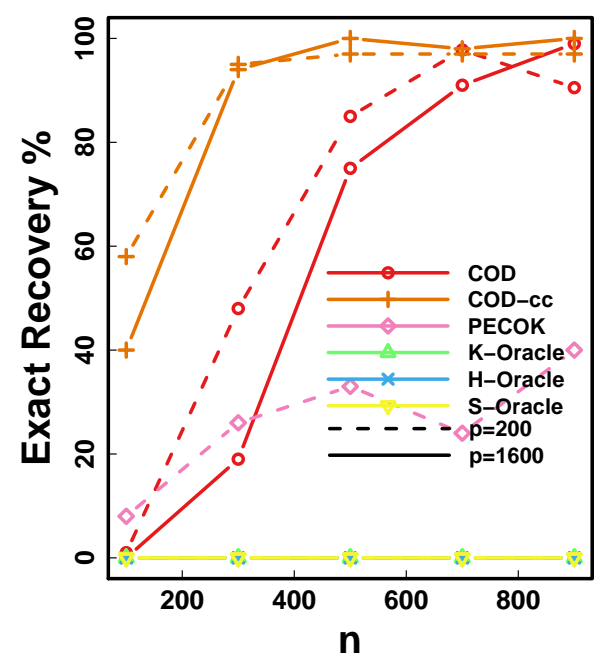

(b) M2



(d) M1P

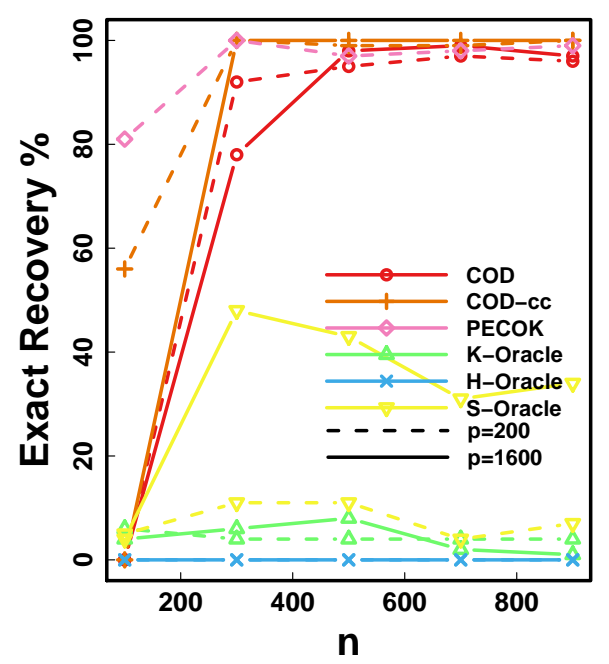


Figure 2: Comparison of Exact recovery percentages across 100 runs. (a) The parameter $K$ in K-means (K-Oracle, medium green lines, triangle points) and $\Gamma$-uncorrected PECOK (UP-Oracle, navy blue lines, square points) are set to the true $K$ while PECOK (light pink lines, diamond points) selects $K$ using our data-splitting criterion. (b) The exact recovery percentages of PECOK are shown under $m=50$ (solid line) and $m=20$ (dash line). All standard errors are smaller than $5 \%$.

(a) With/without correcting for $\Gamma$

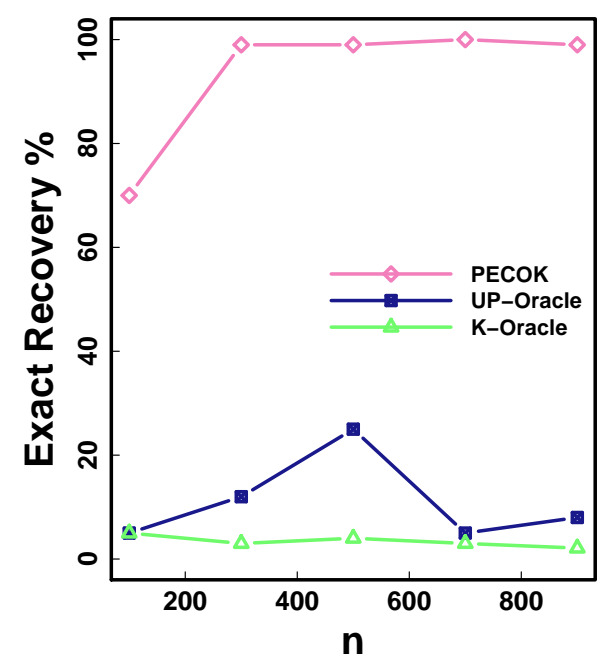

(b) Varying $m$

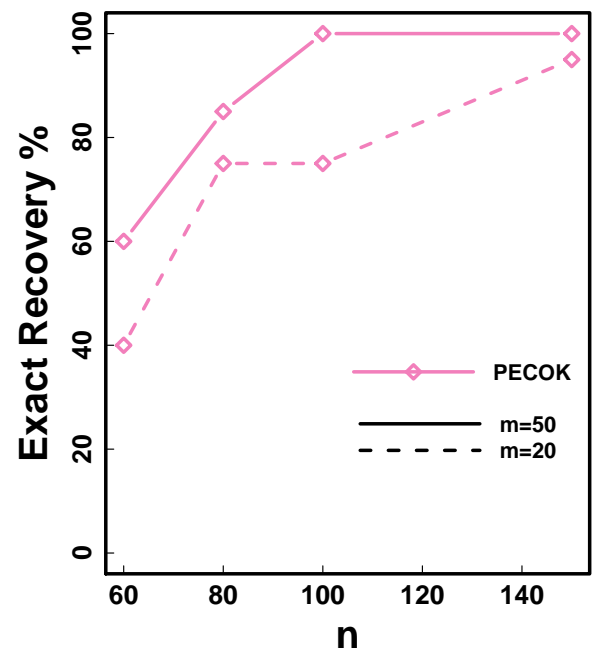

G.5. Partial recovery performance and comparison. Our work is devoted to exact cluster recovery. For completeness, we also assess the performance of our methods in terms of partial cluster recovery, and compare them with existing procedures. We employ the commonly used Adjusted Rand Index (ARI) to compare different methods. The largest ARI value is 1 when the recovered clusters match the truth exactly. The simulated data are the same as those illustrated in Figures 1 and 2. Figure 3 compares the average ARI values under different simulation settings. Our methods, COD, COD-CC, and PECOK, have their ARI measures close to 1 across all simulation settings, outperforming all other methods. Their performance also increases with $n$ as predicted by our theory, while all other competing methods have flat ARI measures even if $n$ increases. Under varying $m$, PECOK yields close to 1 ARI values for small $n=60,80,100,150$.

\section{APPENDIX H: SUPPLEMENTAL MATERIALS FOR THE FMRI EXAMPLE}

Preprocessing. We applied the preprocessing steps suggested by [13], which includes slice timing correction, alignment, registration, normalization to the average $152 \mathrm{~T} 1 \mathrm{MNI}$ template, smoothing with a $5 \mathrm{~mm}$ full-width-half-maximum Gaussian kernel, denoising using the FSL MELODIC procedure, and a high pass filter with a 66 s cut-off. The event-related activation and temporal correlation were removed using general linear models (GLM) for each voxel [3]. Following [9], we extract 180 mean activities within a $10 \mathrm{~mm}$ spheres centered around each of 264 putative functional areas (see Table S2 from [9]).

\section{REFERENCES}

[1] Pierre C Bellec. Concentration of quadratic forms under a bernstein moment assumption. 
Figure 3: (a)-(d) Average ARI values by K-means (K-Oracle, medium green lines, triangle points), $\mathrm{HC}$ (H-Oracle, light blue lines, cross points), spectral clustering (S-Oracle, light yellow lines, up-sidedown triangle points), COD (dark red lines, circle points), COD-CC (light orange lines, plus points), PECOK (light pink lines, diamond points). (e) The parameter $K$ in K-means and $\Gamma$-uncorrected PECOK (UP-Oracle, navy blue lines, square points) are set to the true $K$ while PECOK selects $K$ based on our Data-Spliting criterion. (f) Average ARI values of PECOK are shown under varying $m$.

(a) M1

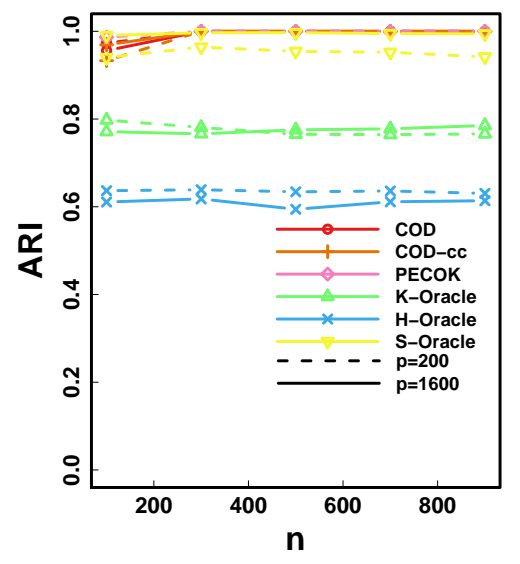

(c) M1S

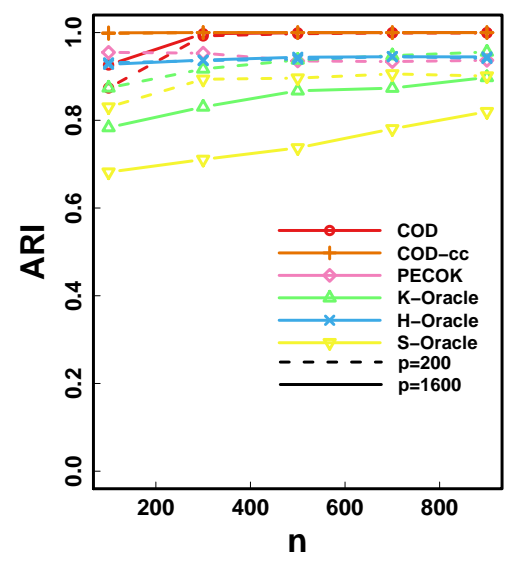

(e) With/without correcting for $\Gamma$

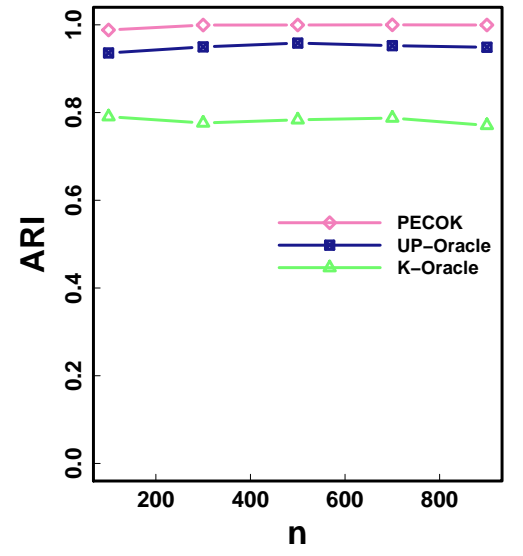

(b) M2

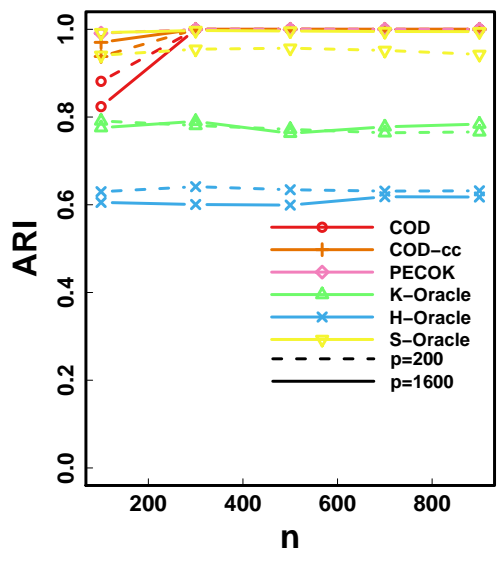

(d) M1P



(f) Varying $m$

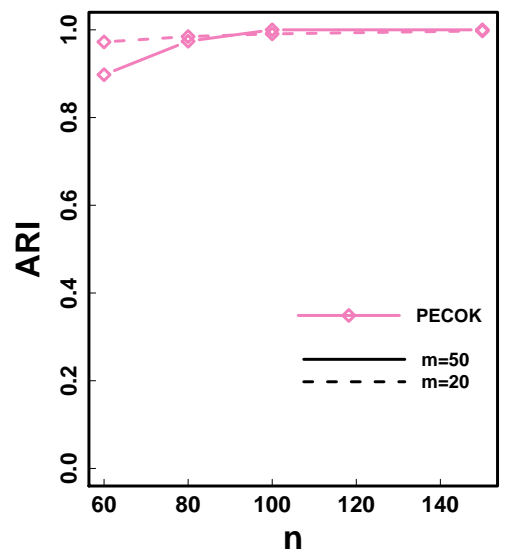


[2] F. Bunea, C. Giraud, X. Luo, M. Royer, and N. Verzelen. Model assisted variable clustering: minimax-optimal recovery and algorithms. Preprint, 2018.

[3] Karl J Friston, Andrew P Holmes, Keith J Worsley, J-P Poline, Chris D Frith, and Richard SJ Frackowiak. Statistical parametric maps in functional imaging: a general linear approach. Human brain mapping, 2(4):189210, 1994.

[4] Vladimir Koltchinskii and Karim Lounici. Concentration inequalities and moment bounds for sample covariance operators. Bernoulli, to appear.

[5] B. Laurent and P. Massart. Adaptive estimation of a quadratic functional by model selection. Annals of Statistics, 28(5):1302-1338, 2000.

[6] Jing Lei and Alessandro Rinaldo. Consistency of spectral clustering in stochastic block models. Ann. Statist., 43(1):215-237, 2015.

[7] Pascal Massart. Concentration inequalities and model selection, volume 1896 of Lecture Notes in Mathematics. Springer, Berlin, 2007. Lectures from the 33rd Summer School on Probability Theory held in Saint-Flour, July 6-23, 2003, With a foreword by Jean Picard.

[8] D. G. Mixon, S. Villar, and R. Ward. Clustering subgaussian mixtures by semidefinite programming. ArXiv e-prints, February 2016.

[9] Jonathan D Power, Alexander L Cohen, Steven M Nelson, Gagan S Wig, Kelly Anne Barnes, Jessica A Church, Alecia C Vogel, Timothy O Laumann, Fran M Miezin, Bradley L Schlaggar, et al. Functional network organization of the human brain. Neuron, 72(4):665-678, 2011.

[10] Mark Rudelson and Roman Vershynin. Hanson-wright inequality and sub-gaussian concentration. Electron. Commun. Probab, 18(82):1-9, 2013.

[11] J. A. Tropp. An Introduction to Matrix Concentration Inequalities. ArXiv e-prints, January 2015.

[12] Roman Vershynin. Introduction to the non-asymptotic analysis of random matrices. arXiv preprint arXiv:1011.3027, 2010.

[13] Gui Xue, Adam R Aron, and Russell A Poldrack. Common neural substrates for inhibition of spoken and manual responses. Cerebral Cortex, 18(8):1923-1932, 2008.

Department of Statistical Science

CORNELl UNIVERSiTy

ITHACA, NY 14853-2601, USA

E-MAIL: fb238@cornell.edu

Department of Biostatistics

and Center for Statistical Science

BROWN UNIVERSITY

Providence, RI 02912, USA

E-MAIL: xi.rossi.luo@gmail.com
LABOratoire de Mathématiques D'Orsay

Univ. Paris-Sud, CNRS, Université PARIs-Saclay

F-91405 Orsay, France

E-MAIL: christophe.giraud@math.u-psud.fr

LABOratoire de MathÉmatiques D'Orsay

Univ. Paris-Sud, CNRS, Université PARIS-SaClay

F-91405 Orsay, France

E-MAIL: martin.royer@math.u-psud.fr

INRA, Montpellier SupAgro, MisteA

UNIV. MONTPELLIER

MONTPELLIER, FRANCE E-MAIL: nicolas.verzelen@inra.fr 
Department of Statistical Science CORNELL UNIVERSITY

ITHACA, NY 14853-2601, USA

E-MAIL: fb238@cornell.edu

Department of Biostatistics

BROWN UNIVERSITY

Providence, RI 02903, USA

E-MAIL: xi.rossi.luo@gmail.com
Laboratoire de MathÉmatiques d'Orsay

Univ. PARIS-Sud, CNRS, Université Paris-Saclay F-91405 Orsay, France

E-MAIL: christophe.giraud@math.u-psud.fr

Laboratoire de Mathématiques d'Orsay

Univ. Paris-Sud, CNRS, Université Paris-Saclay

F-91405 ORSAY, FranCE

E-MAIL: martin.royer@math.u-psud.fr

INRA, Montpellier SupAgro, MisteA

UNIV. MONTPELLIER

MONTPELLIER, FRANCE E-MAIL: nicolas.verzelen@inra.fr 